System-Wide Water Resources Program

\title{
Automated Design of a High-Velocity Channel
}

Jacqueline P. Hallberg

May 2006

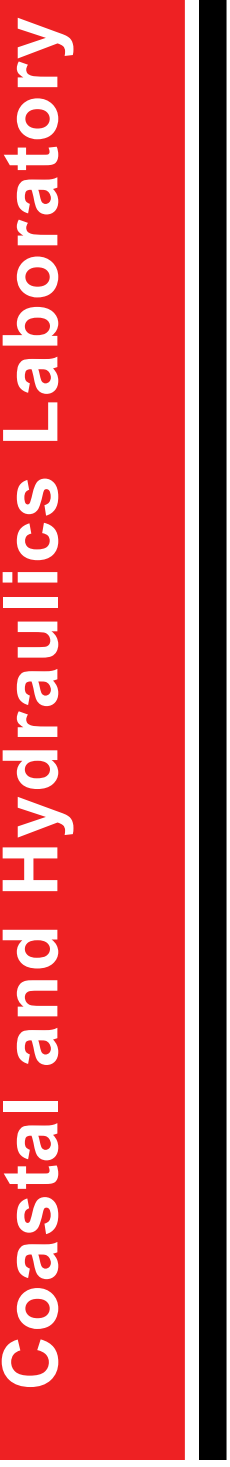

Approved for public release; distribution is unlimited. 


\section{Automated Design of a High-Velocity Channel}

Jacqueline P. Hallberg

Coastal and Hydraulics Laboratory

U.S. Army Engineer Research and Development Center

3909 Halls Ferry Road

Vicksburg, MS 39180-6199

\section{Final Report}

Approved for public release; distribution is unlimited

Prepared for U.S. Army Corps of Engineers

Washington, DC 20314-1000 


\begin{abstract}
Engineering design is a decision-making process. Optimization techniques can be used to insure that better decisions are made. One design of great interest to engineers is that of high-velocity channels used for routing floodwater out of urban areas. In the design of these channels it is very important to avoid such hydraulic phenomena as standing waves, hydraulic jumps, and shocks. These will require higher wall heights and more expense. These channels can be modeled with physical models, but they are expensive and time consuming. To minimize the cost of building and changing the physical models and the time required to perform the study, an automated numerical model can be used to test a range of designs before construction of the physical model. The resulting design can be used as an initial design, which is close to the desired design requiring fewer changes to the physical model, saving time and money.
\end{abstract}

DISCLAIMER: The contents of this report are not to be used for advertising, publication, or promotional purposes. Citation of trade names does not constitute an official endorsement or approval of the use of such commercial products. All product names and trademarks cited are the property of their respective owners. The findings of this report are not to be construed as an official Department of the Army position unless so designated by other authorized documents. 


\section{TABLE OF CONTENTS}

LIST OF TABLES . . . . . . . . . . . . . . . . . . iv LIST OF FIGURES . . . . . . . . . . . . . . . . . . v v CHAPTER

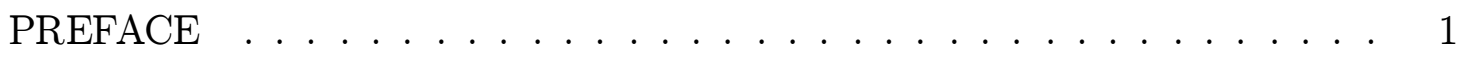

I. INTRODUCTION .......................... 2

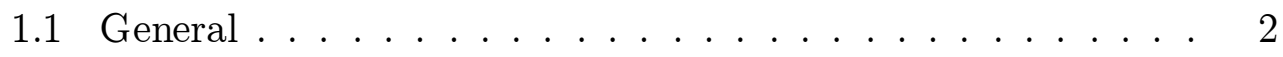

1.2 Objectives ................. . . . . 4

1.3 Scope...................... 5

II. BACKGROUND ................... 6

2.1 Modeling High-Velocity Channels . . . . . . . . . . . 6

2.1.1 Physical Models . . . . . . . . . . . . 6

2.1.2 Numerical Models . . . . . . . . . . . . . . 19

2.2 Literature Review . . . . . . . . . . . . . . . . . . . 20

2.2.1 Aerospace Applications . . . . . . . . . . . . . 20

2.2.2 Groundwater Applications ............ . 21

2.2.3 Surface Water Applications . . . . . . . . . . . . 23

III. METHODOLOGY ................... 30

3.1 Numerical Representation of Physical Problem . . . . . . . . 31

3.2 Objective Function . . . . . . . . . . . . . . . . 33

3.3 Parameter Sensitivity Analysis . . . . . . . . . . . . 34

3.4 Moving the Grid . . . . . . . . . . . . . . . . . . . 34

3.5 Flow Simulation . . . . . . . . . . . . . 36 
CHAPTER $\quad$ Page

3.6 Termination Criteria . . . . . . . . . . . . 36

3.7 Function Evaluation . . . . . . . . . . . . . . . . . . 36

3.8 Design Space Gradient Calculation . . . . . . . . . . . . . . . 38

3.9 Design Variable Update . . . . . . . . . . . . . . . . 39

IV. RESULTS ..................... . . 41

4.1 Numerical Representation of Physical Problem . . . . . . . . 42

4.2 Objective Function . . . . . . . . . . . . . . . . . . . . . . 48

4.3 Parameter Sensitivity Analysis . . . . . . . . . . . 51

4.4 Moving the Grid . . . . . . . . . . . . . . . 68

4.5 Circular Curve Problem . . . . . . . . . . . . . . . . . . . 69

4.6 Super Elevation . . . . . . . . . . . . . . . . . . . 71

4.7 Radius of Curvature . . . . . . . . . . . . . . . 78

4.8 Radius of Curvature and Super Elevation . . . . . . . . 86

V. SUMMARY, CONCLUSIONS, AND RECOMMENDATIONS FOR

FUTURE WORK . . . . . . . . . . . . . . 106

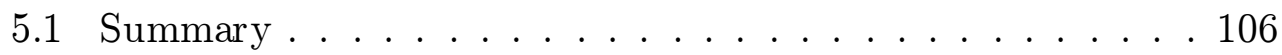

5.2 Conclusions . . . . . . . . . . . . . . . . . 107

5.3 Recommendations for Future Work . . . . . . . . . . 108

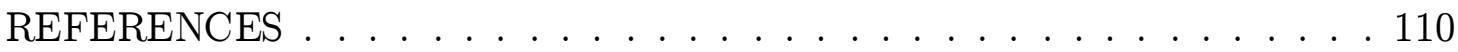
APPENDIX

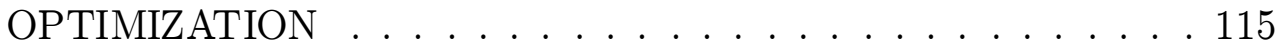




\section{LIST OF TABLES}

TABLE Page

2.1 Scale Relations Between the Physical Model and Prototype . . . . . 8

2.2 Comparison of Optimization Methods [Burg 99] $\ldots \ldots$. . . . . . . . 27

4.1 Parameter Sensitivity Runs . . . . . . . . . . . . . . . 53

4.2 Super Elevation Parameter Sensitivity Objective Function Values 56

4.3 Radius of Curvature Parameter Sensitivity Objective Function Values 61

4.4 Channel Width Parameter Sensitivity Objective Function Values . . 65

4.5 Divider Wall Length Parameter Sensitivity Objective Function Values 66 


\section{LIST OF FIGURES}

FIGURE Page

2.1 Vicinity map of the Walnut Creek Physical Model Study. . . . . . . 10

2.2 Flood of 1958 in Walnut Creek, California . . . . . . . . . . . . . 11

2.3 Channel Layout of Walnut Creek . . . . . . . . . . . . . . . . . 12

2.4 General View of the Physical Model [Davis 87]. . . . . . . . . . . 13

2.5 Standing Waves and Hydraulic Jump near Junction [Davis 87]. . . . 14

2.6 Standing Waves and Overtopping Downstream of Junction [Davis 87]. 16

2.7 Depths Exceeding Wall Heights Downstream of Junction [Davis 87]. 17

2.8 Hydraulic Jump Pushed Out of View [Davis 87]. . . . . . . . . . . 18

3.1 Flow Chart of Method for Optimizing High-Velocity Channel . . . . 32

3.2 Laplacian Smoothing Technique . . . . . . . . . . . . . . . 37

3.3 Residuals at Each Node in the Objective Space . . . . . . . . . . . 40

4.1 The Location of the Study Area. . . . . . . . . . . . . . . . . . 43

4.2 The Design Space for the Optimization Problems. . . . . . . . . . . 44

4.3 The Super Elevation in the Curve. . . . . . . . . . . . . . . . 45

4.4 The Radius of Curvature for the Curve. . . . . . . . . . . . . . . . . 46

4.5 AutoCAD Depiction of Location used to Generate the Mesh. . . . . 47

4.6 The Finite Element Representation and Boundary Conditions. . . . 49

4.7 Water Surface Elevations for the Physical and the Numerical Models. 50 
4.8 Objective Space for Optimization. . . . . . . . . . . . . . 52

4.9 Bed Elevations for No(left), 2' (center), and 6' (right) Super Elevation. 55

4.10 Depth Profile in Area1 and Area2 for Super Elevation Test Cases. . 57

4.11 Resulting Depths for the Super Elevation Test Cases. . . . . . . . . 58

4.12 Bed Elevations of the Original Curve, 350' Radius, and 400' Radius. 59

4.13 Depth Profiles in Area1 and Area2 for Radius of Curvature. . . . . 60

4.14 Bed Elevations for Channel Widths of 20' (left) and 25' (right). . . 62

4.15 Water Depth Profiles in Area1 and Area2 for Width Test Cases. . . 63

4.16 Depths for Channel Widths of 20' (left), 23' (center), and 25' (right). 64

4.17 Water Depth Profiles in Area1 and Area2 for Divider Wall Test Cases. 67

4.18 Layout of a Circular Curve with Spirals . . . . . . . . . . . . . . 70

4.19 Initial Mesh for the Circular Curve Optimization Problem. . . . . . 72

4.20 Objective Function and Design Space for the Circular Curve Problem. 73

4.21 Objective Function Values versus Radius of Curvature. . . . . . . . 74

4.22 Layout of Curve Including Bounds for Points of Tangency. . . . . . 75

4.23 Initial Grid for the Super Elevation Problem. . . . . . . . . . . . . . 77

4.24 The Design Space for the Super Elevation Problem. . . . . . . . . . 79

4.25 The Objective Area for the Super Elevation Problem. . . . . . . . . 80

4.26 Design Parameter versus Design Iteration. . . . . . . . . . . . . 81

4.27 Layout and Bed Elevations of the Improved Design. . . . . . . . . . 82

4.28 Depth Profiles for Initial Design and Improved Design. . . . . . . . 83

4.29 Depth Profiles for Physical Model Design and Improved Design. . . 84

4.30 Design Variable versus Objective Function. . . . . . . . . . . 85

4.31 Initial Grid for the Radius of Curvature Problem. . . . . . . . . . . 87

4.32 The Design Space for the Radius of Curvature Problem. . . . . . . 88

4.33 The Objective Area for the Radius of Curvature Problem. . . . . . 89 
4.34 Design Variable versus Design Iteration. . . . . . . . . . . . 90

4.35 Layout of the Improved Design. . . . . . . . . . . . . . . . 91

4.36 Depth Profiles for Initial Design and Improved Design. . . . . . . . 92

4.37 Depth Profiles for Physical Model Design and Improved Design. . . 93

4.38 Design Variable versus Objective Function. . . . . . . . . . . . . 94

4.39 Initial Grid for the Radius of Curvature and Super Elevation Problem. 96

4.40 Design Space for the Two Design Variable Problem. . . . . . . . . 97

4.41 The Objective Area for the Two Design Variable Problem. . . . . . 98

4.42 Design Variable versus Design Iteration. . . . . . . . . . . . . 99

4.43 Layout of the Improved Design. . . . . . . . . . . . . . . 100

4.44 Depth Profiles for Initial Design and Improved Design. . . . . . . . 101

4.45 Depth Profiles for Physical Model Design and Improved Design. . . 102

4.46 Design Variable versus Objective Function. . . . . . . . . . . . . 103

4.47 Design Variable versus Objective Function. . . . . . . . . . . . . 104

4.48 Design Variable versus Objective Function. . . . . . . . . . . 105 


\section{PREFACE}

This report is a thesis in partial fulfillment of the requirements for the degree of Master of Science from Mississippi State University.

This report was written by Jacqueline P. Hallberg, Coastal and Hydraulics Laboratory (CHL), U.S. Army Engineer Research and Development Center (ERDC). Dr. David Huddleston (Mississippi State University) served as thesis director. Drs. Charlie Berger and Stacy Howington served as technical advisors and thesis reviewers. Cheryl P. Boyd served as thesis reviewer. Several people contributed to the success of this research: Dr. Clarence Burg, Dr. Richard L. Stockstill, Chuck H. Tate, Jr., Van E. Stewart, Dewayne B. Fuller, Glenn Davis, and John F. George.

This report was funded by the System-Wide Water Resources Program (SWWRP). Dr. Steven L. Ashby is Program Manager of SWWRP. Thomas W. Richardson is the Director of CHL and Dr. William D. Martin is Deputy Director. Earl Edris is the Branch Chief for the Hydrologic Systems Branch.

COL James R. Rowan was Commander and Executive Director of ERDC. Dr. James R. Houston was Director. 


\section{CHAPTER I \\ INTRODUCTION}

\section{$1.1 \quad$ General}

The population of Los Angeles County had grown to more than a half million residents by 1910. Few had experienced the prior overflows of the Los Angeles River and even fewer understood the need for flood control, but they soon would. On February 18, 1914, shortly after midnight, rain began to fall on the already saturated ground. It rained for three days delivering more than 19 inches of rain in some locations. Los Angeles received over 7 inches, including 1.5 inches in an hour. The Los Angeles River, the San Gabriel River, the Rio Hondo, and Ballona Creek all overflowed. Flood discharge was 31,400 cubic feet per second, which is equal to the normal flow of the mighty Colorado River. 11,763 acres in Los Angeles were inundated by floodwaters. The flooding destroyed over 100 roads and washed out 35 bridges. Rail service was suspended and communication with the outside world was interrupted for nearly a week. Four million cubic yards of silt were dumped into Los Angeles and Long Beach harbors by the flood-swollen Los Angeles River, rendering some channels unnavigable. Damage throughout the county was estimated at $\$ 10$ million (\$151 million in 1995 dollars). Later, county assessors calculated that flooding had reduced the value of property in the county by $\$ 20$ million. The devastating flood of 1914 clearly demonstrated that the Los 
Angeles River could no longer be allowed to wander at will. It must be controlled. [Gumprecht 97]

The design of structures to control waterways such as the Los Angeles River is a major concern for engineers. The options for flood control in urban areas, however, are limited. A large fraction of the ground surface is paved causing concentrated flood flow peaks. It is not feasible to build a reservoir in downtown Los Angeles or modify the landscape to control this flow. A practical method of routing the water through the urban areas is via the use of high-velocity channels. High-velocity channels offer the capability to carry supercritical flow.

A lot of details must be considered when designing flood control structures. Engineering design is a decision-making process. Optimization techniques can aid in ensuring that better decisions are made concerning the design. An area of engineering design that can benefit from optimization techniques is the design and modification of high-velocity channels essential for the routing of floodwater through urban areas. The proper design of new channels and re-design of existing channels is required to avoid such things as bank erosion, damaged equipment, increased operating expenses, flooding, and higher construction costs. Physical models are very useful as a tool to determine appropriate designs of flood channels to meet certain site-specific criteria, but initial design and modifications of physical models are very costly in both time and money. Due to the time and cost constraints of physical models, it is not practical to examine a wide range of designs. This could result in hydraulic performance that is only acceptable over a limited range. The ideal scenario is to build the initial physical model as close to the final design as possible. This can be accomplished by using an automated hydraulic design program to produce a better design prior to the building of the physical model which will reduce both design time and cost. 
Burg [Burg 99] has built such a capability. He explored numerous optimization methods to determine those most suitable for problems involving high-velocity channels. Burg's work demonstrates an optimization strategy integrated with the HIVEL2D analysis code for design optimization of simple two-dimensional test cases. HIVEL2D, a two-dimensional numerical model that solves the shallow water equations, is a proven tool for modeling these types of problems [Berger 95]. Examples presented by Burg, however, were represented by simple geometries. The examples deal with flume type problems, as well as super elevation in circular bends and embedded bodies such as bridge piers. The application of this technique to an urban flood-control channel, previously designed through an experimental study, is the concern of this investigation. Application to such a "real-world" problem will provide an assessment and demonstration of the utility of the design method.

\subsection{Objectives}

The objective of this work is to assess the practicality of using the optimization technique developed by Burg to aid in the design of a realistic high-velocity channel. Since the test cases used by Burg were represented by simple geometries and did not address the more complex features of channels, the steps required to address more complex problems must be established. Therefore, the procedure for applying design optimization techniques to "real-world" problems will be determined by this research. The desired outcome of the research is to apply Burg's technique to a "real-world" problem and to develop a procedure for similar future applications. 


\subsection{Scope}

A numerical model of the Walnut Creek physical model is developed and simulation results are compared to the physical model results. A series of model parameters are tested to determine the model sensitivities. This reduces the number of parameters to only those that have a major impact on the design. The results, along with engineering judgment, are used to explore and determine the appropriate parameters to be optimized and to explore the applicability of possible objective functions to be used in the model. Once the appropriate design

parameters and objective function are determined, the design is automated to yield a better hydraulic design for the given constraints. Walnut Creek was used as an evaluation case since the design of this hydraulic structure was extensively analyzed via an experimental project. [Davis 87] 


\section{CHAPTER II}

\section{BACKGROUND}

\subsection{Modeling High-Velocity Channels}

High-velocity channels are used for drainage in urban regions, since urban sprawl increases rainfall runoff due to altered land use. Flood control channels are designed and built to safely manage the anticipated hydrologic load. The desire is to minimize the water's time of residence in the urban area. The channels are designed to carry supercritical flow to reduce the water depths and the required route. Structures, such as bends, transitions, and confluences cause flow to choke, form jumps, and/or form standing waves. These hydraulic conditions generally necessitate higher walls, bridges, and other costly containment structures. A poorly designed channel can cause bank erosion, damaged equipment, increased operating expense, and reduced efficiency [Berger 95]. Furthermore, crossings may be washed out, and the town may flood. To improve the design of channels, engineers use models to reproduce the channels and run a suite of tests to determine the functionality of the channels under selected hydraulic conditions. The two ways of modeling these channels are with physical models and with numerical models.

\subsubsection{Physical Models}

Numerical modeling of high-velocity channels has emerged as a tool to augment physical models. Physical models are not as popular as they once were, but they are still very useful in channel design due to the limitations found in numerical models. 
One such limitation of shallow water models is that they are typically hydrostatic. Physical models can model things such as non-hydrostatic flow at the tail of a bridge pier that a shallow-water numerical model is not capable of modeling. Physical models are extremely expensive to build, costing on the order of $\$ 150,000$ per study and are time consuming requiring an average of three months to complete [Stockstill 00]. Changes to the physical model require a "cut and try" technique that involves tearing down the unwanted sections of the channel and rebuilding them with the new desired design [Soulis 92]. Each modification on average costs $\$ 30,000$ and take a minimum of 1 month to complete. Though physical models can reproduce details of actual hydraulic structures, they are subject to the limitation of scale modeling. It is impossible to reproduce the physical problem to scale. The problem is usually scaled down to a more cost efficient and manageable size. For instance, a physical model constructed on a scale of 1:25 means that 1 foot of model is equal to 25 feet of prototype. Scaling the model to a more reasonable size reduces the space and materials required to build the physical model. The equations of hydraulic similitude, based on Froudian relations, are used to express mathematical relations between the dimensions and hydraulic quantities of the model and prototype. The bed slope of the physical model is adjusted to account for the difference in the roughness of the model material and the prototype. Table 2.1 gives the scale relation between the physical model and prototype.

A physical model study conducted at the Waterways Experiment Station of the Walnut Creek Flood-Control Project in Contra Costa County, California, is considered for testing the applicability of Burg's techniques to an actual problem. The physical model study was a 1:25 reproduction of approximately $1,084 \mathrm{ft}$ of the San Ramon Bypass Channel, $730 \mathrm{ft}$ of the Walnut Creek Channel upstream from the junction, and $640 \mathrm{ft}$ of the Walnut Creek Channel downstream from 
Table 2.1: Scale Relations Between the Physical Model and Prototype

\begin{tabular}{ccc}
\hline Characteristic & Dimension (terms of length) & Model:Prototype \\
\hline Length & $L_{r}=\mathrm{L}$ & $1: 25$ \\
Area & $A_{r}=L_{r}^{2}$ & $1: 625$ \\
Velocity & $V_{r}=L_{r}^{\frac{1}{2}}$ & $1: 5$ \\
Time & $T_{r}=L_{r}^{\frac{1}{2}}$ & $1: 5$ \\
Discharge & $Q_{r}=L_{r}^{\frac{5}{2}}$ & $1: 3,125$ \\
Roughness Coefficient & $N_{r}=L_{r}^{\frac{1}{6}}$ & $1: 1.71$
\end{tabular}

the junction (Figure 2.1). The project was aimed at making improvements to the channel to provide flood protection to about 6,670 acres in the floodplain at and below the city of Walnut Creek. The site contains several hydraulic structures including circular curves with super elevation and transition spirals, a divider wall, a contraction, and a confluence. Figure 2.3 shows the layout of the channels and Figure 2.4 shows a general view of the physical model.

A series of floods had necessitated re-evaluation of the Walnut Creek FloodControl channel design. In 1955, Walnut Creek had a peak discharge of 11,000 cfs and San Ramon Creek had a peak discharge of 6,900 cfs. As a result, 3,500 acres flooded and 1,000 homes and 50 businesses were affected with the damage estimated to be $\$ 1$ million. A flood in 1958 had similar results. The peak discharge of Walnut Creek was 12,200 cfs. 3,400 acres were flooded and 140 businesses and 800 homes were damaged as shown in Figure 2.2. The estimated cost of damages was $\$ 1 \frac{1}{2}$ million. In 1960, the Flood Control Act was passed which authorized the Walnut Creek Basin Project. Smaller floods followed in 1962 and 1963. The 
100-year event was estimated in 1964 to be 18,000 cfs. Design improvement for the Walnut Creek Basin soon began. In the late 1970s, however, flood problems continued in the San Ramon, Las Trampas, and Walnut Creeks due to the small capacity of the existing channels. Urbanization had decreased infiltration and increased runoff in the area. In 1982, peak flow of 7,000 cfs occurred in San Ramon Creek causing flow to approach the top of the creek banks and lap at bridges. The solution was determined to be a bypass channel connecting San Ramon Creek and Walnut Creek. The purpose of the physical model study was to determine the adequacy of the channel during 100-year frequency flow conditions and to develop modifications to increase the hydraulic capacity and improve flow conditions. That is, minimize cross-waves generated by the curves and the junction.

The main focus of the study was the length of the divider wall extension at the junction. Channel junctions are one of the more important hydraulic problems, because as flows from smaller channels are combined standing waves may be produced necessitating increased wall heights in the vicinity of the junction. Also, hydraulic jumps may form in one or both of the channels if the divider wall is not correctly located or scaled. Figure 2.5 shows a hydraulic jump and standing waves in the vicinity of the junction. Several divider wall extension lengths were modeled under various flow conditions. Testing of divider wall extensions are simpler than testing other parameters because it only requires that sections of different lengths be placed in the model and oriented by the engineer. There is no need for tearing down and rebuilding the model. Other areas of interest were the width of the San Ramon Bypass Channel and improved channel alignment at the junction.

In 1984, the estimated 100-year flood event for the Walnut Creek Flood-Control Project was 22,000 cubic feet per second. Currently, the 100-year flood event has not changed from its estimated value in 1984. Because the San Ramon Bypass 


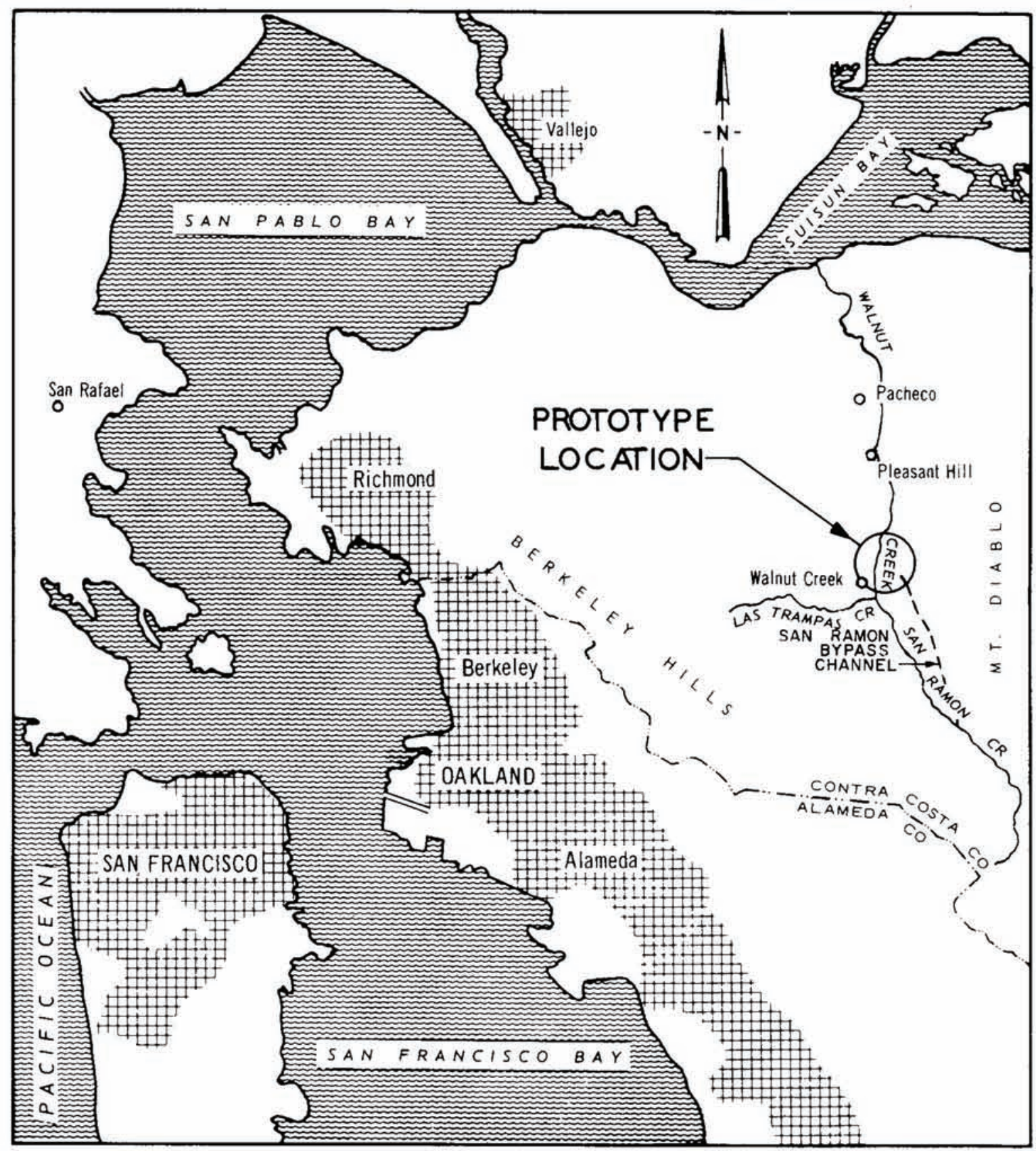

SCALE IN MILES

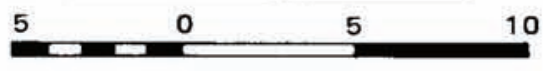

Figure 2.1: Vicinity map of the Walnut Creek Physical Model Study. 

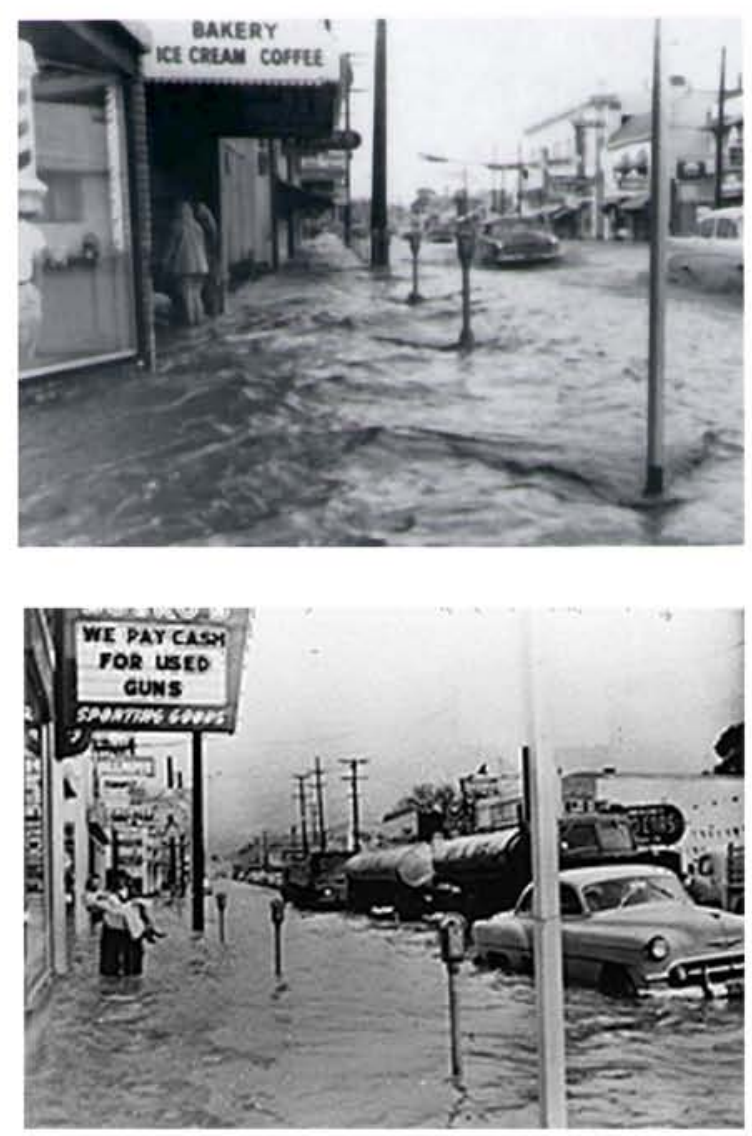

Figure 2.2: Flood of 1958 in Walnut Creek, California

Channel connects San Ramon Creek and Walnut Creek and bypasses the junction between Las Trampas Creek and San Ramon Creek which forms Walnut Creek, the amount of flow in the San Ramon Bypass Channel effects the amount of flow in Walnut Creek. Two discharge conditions were considered. The first condition allowed for maximum flow in the San Ramon Bypass Channel with the concurrent flow set for Walnut Creek. The second condition allowed for the maximum flow in Walnut Creek with the concurrent flow set for the San Ramon Bypass Channel. In prototype, increasing discharges caused standing waves to develop downstream of 


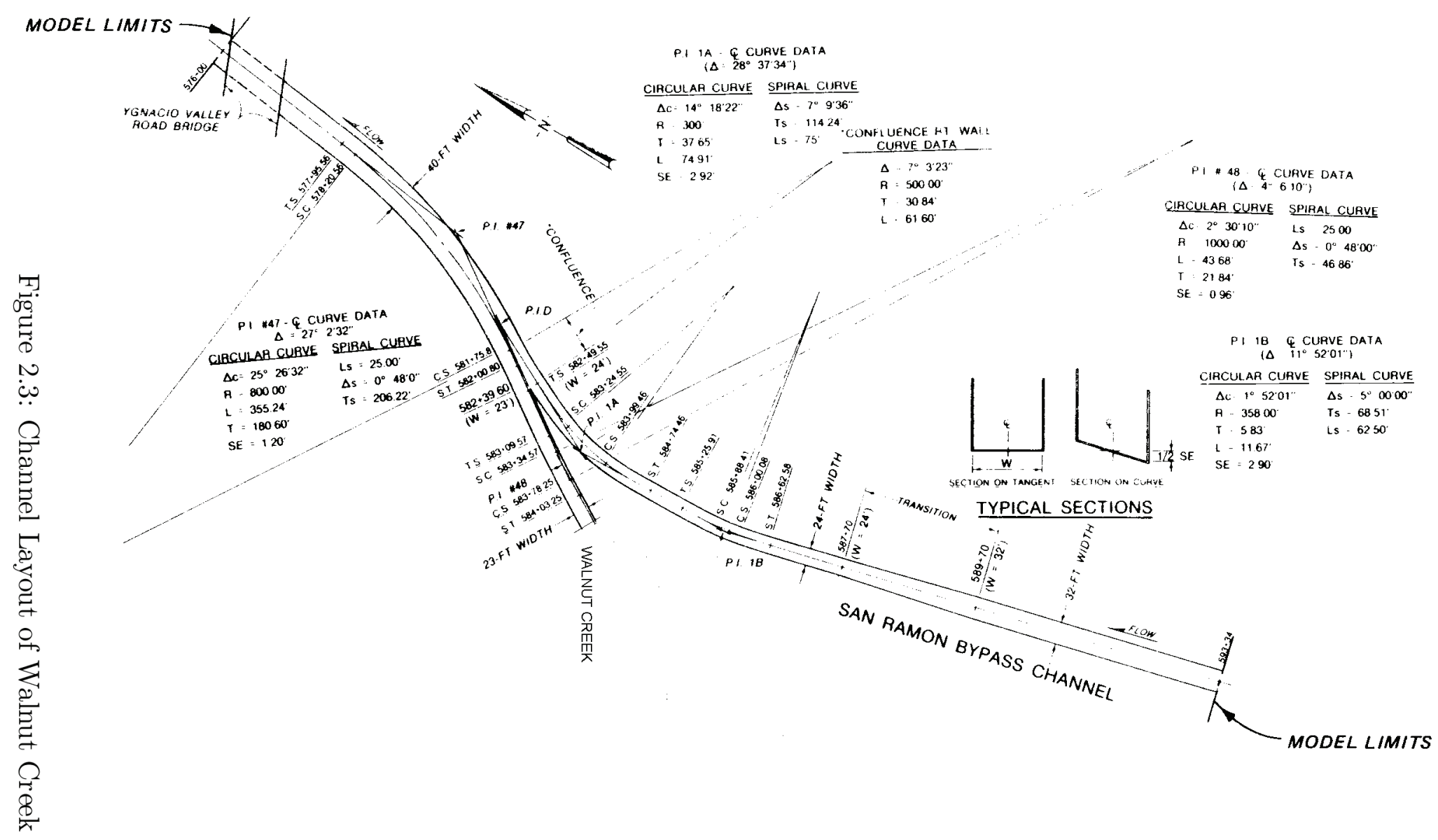

PLAN VIEW 


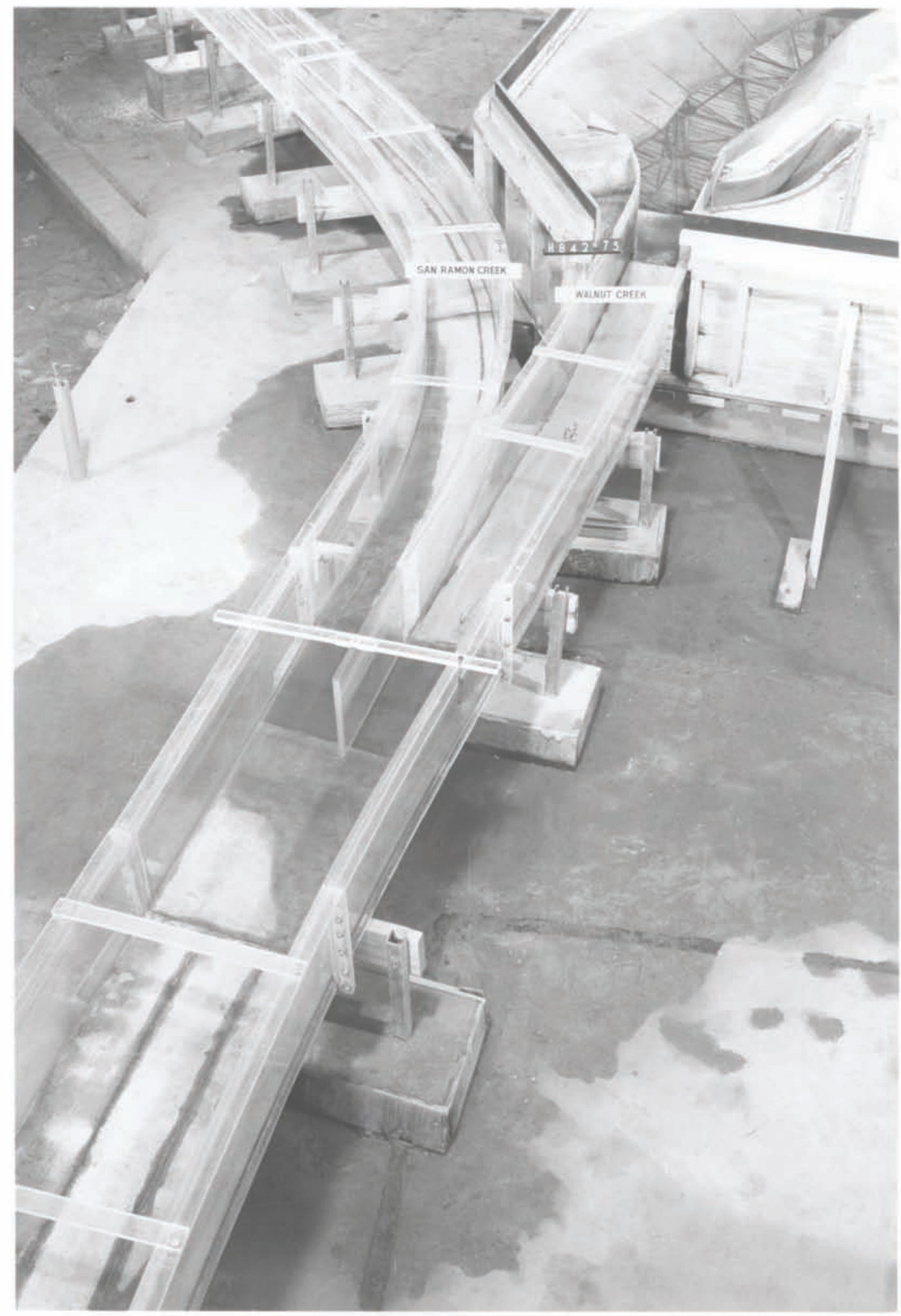

Figure 2.4: General View of the Physical Model [Davis 87]. 


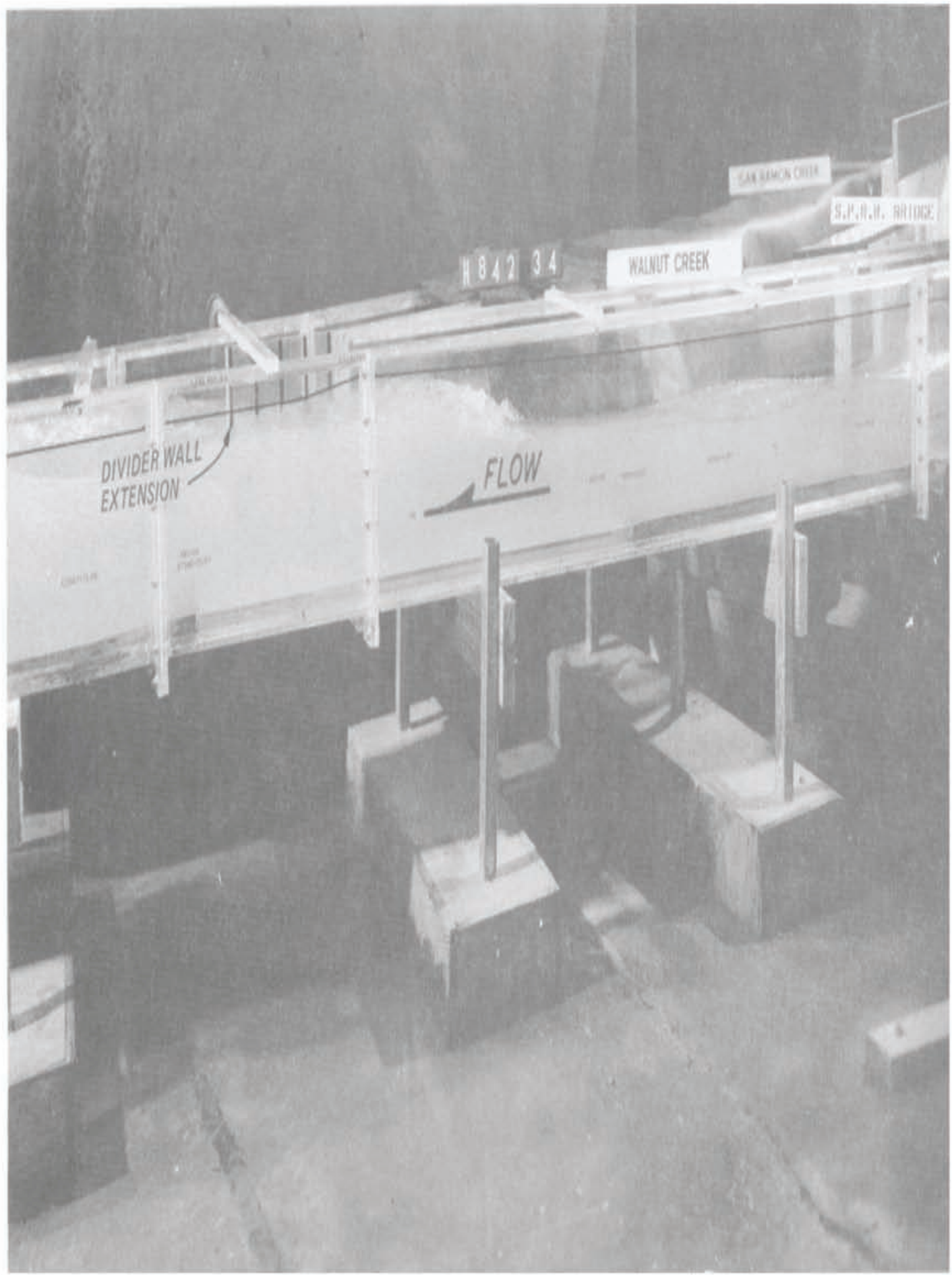

Figure 2.5: Standing Waves and Hydraulic Jump near Junction [Davis 87]. 
the confluence and extend several hundred feet due to the poor channel alignment as illustrated in Figure 2.6. Water depth exceeded the wall heights at several points downstream from the junction as illustrated in Figure 2.7. Adding a divider wall extension caused flow to choke and form a hydraulic jump in Walnut Creek just before the junction that spread upstream as the length of the divider wall was increased. Figure 2.8 shows the jump pushed upstream in Walnut Creek about 70 feet from the junction. The longer the divider wall extension, however, the smoother the water surface downstream from the junction. The physical model study concluded that a 40-foot long divider wall extension should be added, the width of San Ramon Bypass Channel should be decreased, and the channel alignment at the junction should be improved [Davis 87].

The number of designs that can be explored by a physical model is limited by the construction time and cost, though defining the details of the model requires exploration of a vast range of alternatives. A numerical model such as HIVEL2D can be used to assess the design computationally before construction of the physical model begins and to screen alternatives. Using an automated hydraulic design package would accelerate this screening process and lead to an improved initial physical model thus reducing the time spent on the physical model. This would allow for explor ation of more design alternatives in a shorter length of time resulting in a more cost-effective solution. Though the HIVEL2D numerical model cannot show such hydraulic phenomena as non-hydrostatic flow at the tail of a bridge pier, which would require a physical model or more complex computational model, it can give an improved initial design and reduce the number of modifications required. This is a restriction on the hydraulics only not the optimization. 


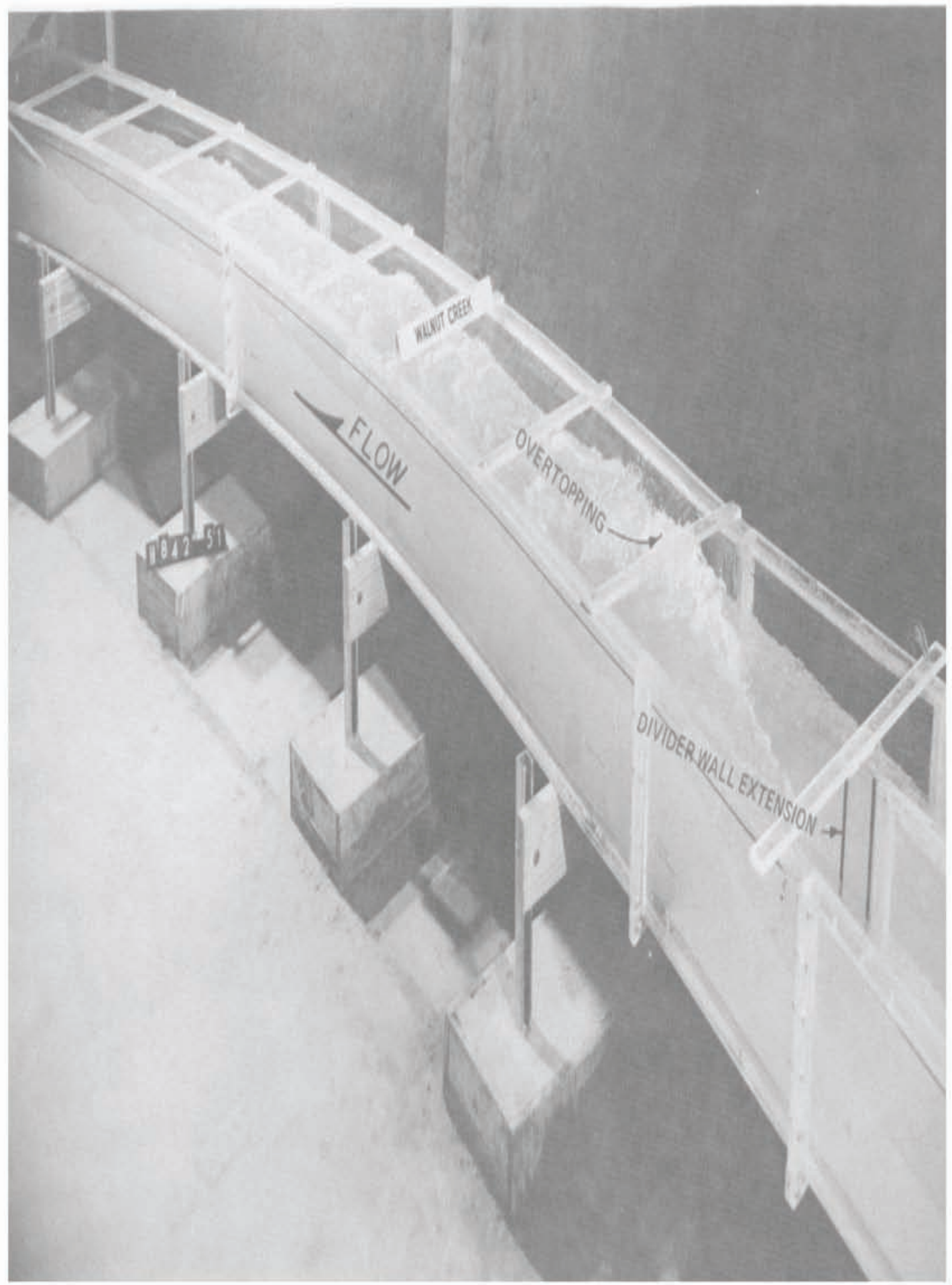

Figure 2.6: Standing Waves and Overtopping Downstream of Junction [Davis 87]. 


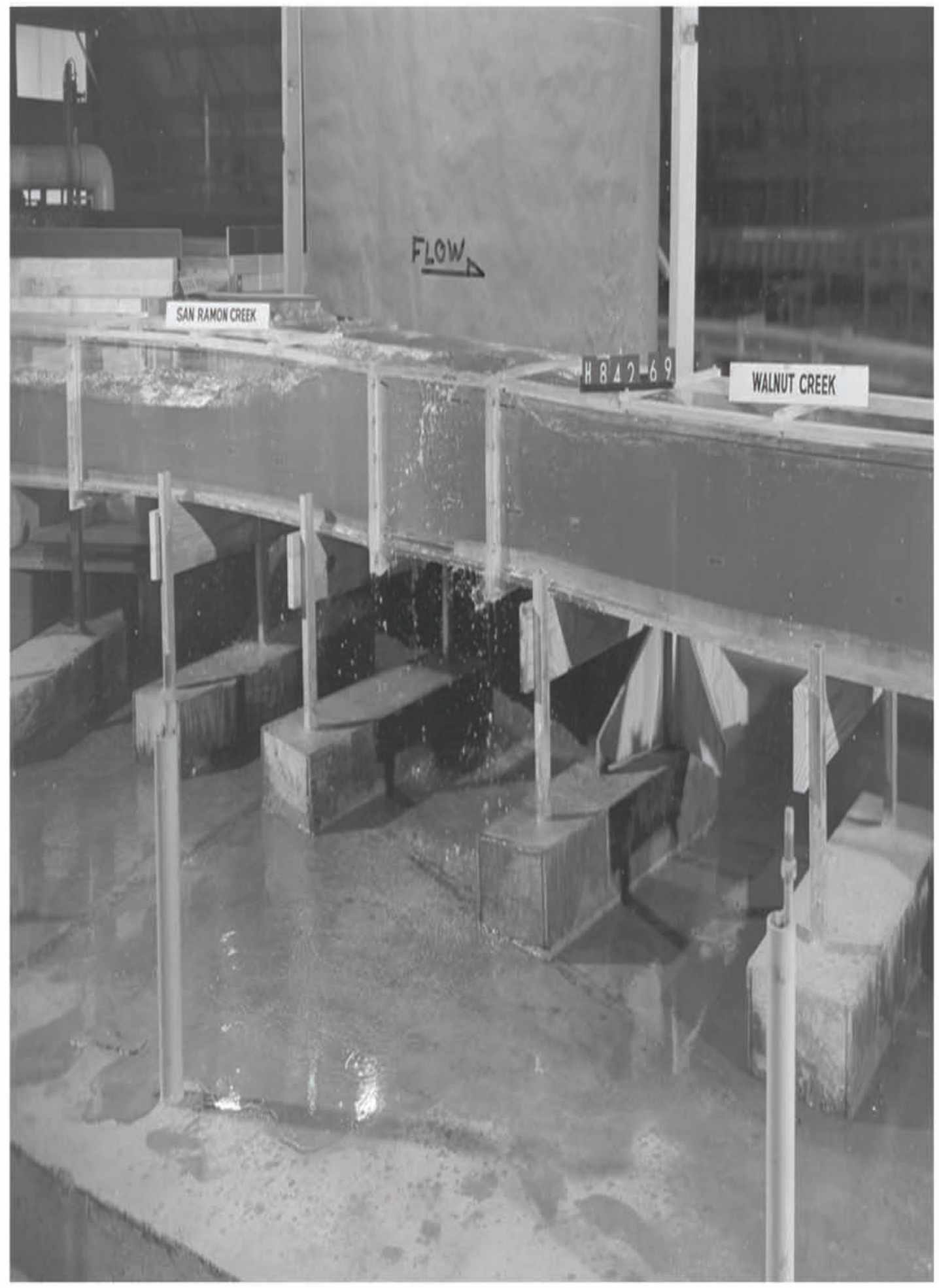

Figure 2.7: Depths Exceeding Wall Heights Downstream of Junction [Davis 87]. 


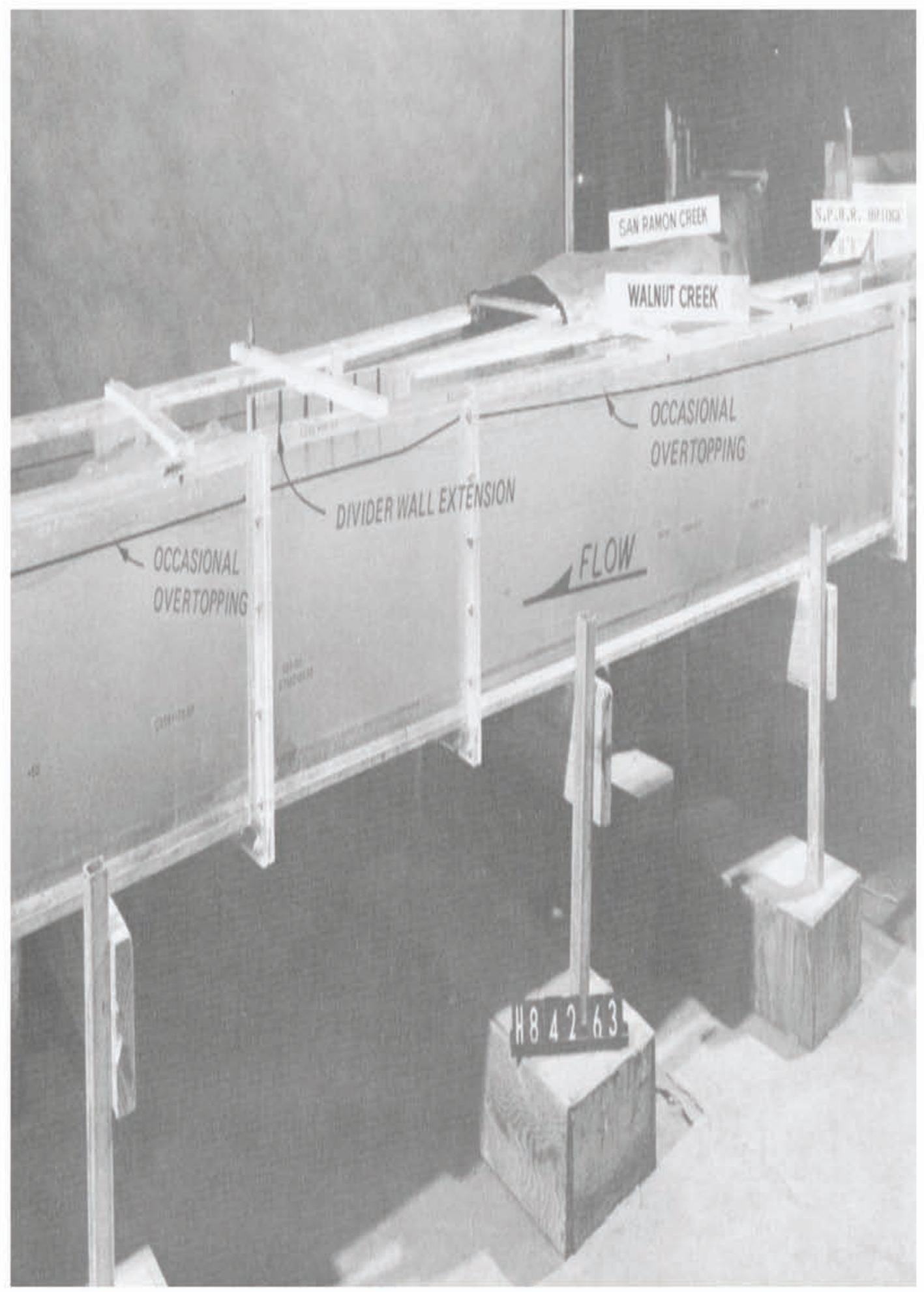

Figure 2.8: Hydraulic Jump Pushed Out of View [Davis 87]. 


\subsubsection{Numerical Models}

Numerical simulations are advantageous because they are relatively fast and inexpensive, which allows for more changes and testing of more parameters compared to physical modeling. Since the vertical accelerations are usually small, the primary basis for simulating flow in high-velocity channels is the shallow water equations. The shallow water equation models, however, are limited by the assumptions made in the equations and by the limitations of the computer. The size of the computer can limit the amount of spatial resolution that can be used to represent the problem.

There are numerous numerical models for solving open-channel flow. For example, Zhou and Stansby [Zhou 99] developed a model to predict the hydraulic jump in a straight open channel. Guillou and Nguyen [Guillou 99] present a technique for solving the two-dimensional shallow water problems based on a finite volume technique. The model chosen for this study is a finite element model, HIVEL2D, developed by Berger and Stockstill [Berger 95].

HIVEL2D is appropriate for this work because of its capability to model supercritical, subcritical, and transition flow. Since the objective of this study is to identify non-smooth flow or sloshing, a model of at least two-dimensions is required.

The vertical accelerations for this problem are negligible compared to the effects of gravity. HIVEL2D solves the shallow water equations in conservative form, which conserves momentum and allows for the correct calculation of the shock speed and location. Three-dimensional models can be used for this simulation, but they are more costly and have capabilities that are not required to solve this problem. The bed slope in the Walnut Creek problem is geometrically mild though hydraulically steep, which meets the assumption made by HIVEL2D. HIVEL2D is designed for 
solving problems with rapidly varying flow and is appropriate for the Walnut Creek problem.

\subsection{Literature Review}

Since optimization can be a powerful tool for engineers during the design process, optimization techniques are used in a vast range of engineering areas. Numerous optimization techniques have been applied to engineering problems in the areas of aerospace, groundwater, and surface water.

\subsubsection{Aerospace Applications}

Mäkinén et al. [Mäkinén 99] applied optimization to a multi-objective, multidisciplinary design optimization of a two-dimensional airfoil. Genetic algorithms were used to obtain an approximation for the Pareto set of optimal solutions. This work is not traditional because it optimizes more than one objective function of more than one discipline. The two objective functions are the drag coefficient with constraints of the lift coefficient above a given value with CFD analysis solvers based on finite volume discretizations of the inviscid Euler equations and the integral of the transverse magnetic radar cross section over a given sector. Several non-dominated designs were obtained.

Design sensitivity analysis is discussed in Hou et al. work [Hou 94]. This method is applied to aerodynamic problems. A derivation of the aerodynamic sensitivity equations is presented that uses the existing formats of implicit algorithms for solving the Euler equations. The derived sensitivity equations are efficiently solved with a direct equation solver for small-scale problems.

Optimization can be used to determine airfoil design such that pre-specified design criteria are met. Soemarwoto et al. [Soemarwoto 99] explored the area of 
aerodynamic airfoil shape optimization. This work applied the adjoint operator approach, utilizing a compressible inviscid flow model based on the Euler equations and a compressible viscous flow model based on Reynolds-averaged Navier Stokes equations. The model solves constrained transonic aerodynamic design (pressure drag reduction) problems.

The emphasis on reduction of design cycles, time, and cost in design of commercial aircraft has sparked a renewed interest in design optimization in aerodynamic structures and aeroelastics. In their work, Melvin et al. [Melvin 99] use the TRANAIR code to optimize design. TRANAIR is a two- and threedimensional full potential flow code with directly coupled strip boundary layer and is capable of handling complex geometries through solution adaptive local grid refinement. The sensitivity method is preferred to the adjoint method because some second-order information is naturally and inexpensively available in the sensitivity method. The problem is solved using Newton's method.

\subsubsection{Groundwater Applications}

Optimization methods are also very useful for solving groundwater problems. Townley et al. [Townley 85] apply present computational algorithms to steady and transient models for groundwater flow. The aquifer storage coefficients, transmissivities, distributed inputs, and boundary values may all be uncertain. The discrete derivation of the adjoint method is used to calculate the derivative of a function with respect to the parameters of the transient numerical flow models. The explicit calculation of sensitivities and the calculations of the gradient of the objective function in the inverse problem are calculated with the adjoint method. A Gauss-Newton line search procedure is used to relate the second derivative in the 
direction of a search to the projection of the sensitivity matrix onto the direction of search.

Adjoint state equations are used in Sun et al. [Sun 92] work for the stochastic partial differential equations relating transient head and log hydraulic conductivity perturbations. The model reliability is evaluated through the variance estimate method using adjoint sensitivity analysis and the cokriging estimate. A stochastic inverse procedure for transient groundwater flow was developed. By using the adjoint state equation and solving for the expected head, all elements of the covariance matrix can be calculated based on the first-order approximation.

Guan et al. [Guan 99] use optimization to design a pump-and-treat groundwater remediation system. A new computational procedure called a progressive genetic algorithm is used to minimize the total cost of the pumpand-treat system while defining the locations and extraction or injection rate of the wells. Constraints of concentration, velocity, and injection and extraction balance were considered. These techniques proved reliable and robust for the given problem.

Optimization techniques have also been used to locate wells for monitoring purposes. In work done by Storck et al. [Storck 97], the flow and transport simulations are passed to an optimization model. The optimization problem is solved by simulated annealing. The problems consist of three conflicting objectives; maximum detection probability, minimum cost (number of wells), and minimum volume of contaminated groundwater at the time of detection. Application of this model to groundwater problems show that the trade-off curves for the objectives based on too few realizations over predicts monitoring performance. 


\subsubsection{Surface Water Applications}

Atanov et al. [Atanov 98] developed an optimization method for minimizing water-level fluctuations in an open-channel controlled by pumping stations on both ends. The problem was a variational problem to determine the optimum flow

control, given constraints at the opposite end. A global measure of the water level deviation away from a desired water level integrated in space and time was used as the objective function. The goal was to minimize deviations while satisfying governing equations and boundary conditions. The problem was solved using a direct flow solver and a conjugate determination of the Lagragian multiplier solved backward in time.

Hsu et al. [HsuM 99] used a vertical (laterally averaged) two-dimensional model of a branched estuarine river system to determine friction and turbulent diffusion/dispersion coefficients. The coefficients affect the calculations of the surface elevation velocity and salinity distribution.

Soulis et al. [Soulis 92] designed open-channel expansions and contractions to give specified distributions of depth. Prescribing depth-velocity values can avoid boundary layer separation and cavitation. The usual design process involves the use of physical and/or numerical models to resolve the fluid properties adequately. The combined effects of physical and numerical approaches can lead to designs acceptable from an engineering point of view. Computer-aided design will improve the performance of any hydraulic structure while shortening the amount of human effort needed for such a design. The authors developed a general numerical method of design for two-dimensional channel expansions and contractions with prescribed depths and velocities along the channel walls using a finite volume analysis code. The process iterates between the direct solution and an inverse solution. For 
a typical problem the procedure should be incorporated 20-30 times in order to converge to an acceptable geometry. This procedure essentially reverses the roles of the independent variables $\mathrm{x}$ and $\mathrm{y}$ (spatial variables) and the dependent variables h, p, and q (flow variables) at specific locations. The application of this method is limited and not as general as the method used in this work.

Mohammadi et al. [Mohammadi 99] used an optimal shape design and unstructured mesh deformations, automatic differentiation for the gradient computation, and mesh adaption by metric control in two-dimensions. For a CADfree design, the only geometrical entity available during optimization should be meshed. The model uses the reverse mode of automated differentiation to produce gradients of discrete operators. The mesh is part of the optimization procedure and a function of the solution. This avoids mesh dependencies in optimization and the direct problem.

In work done by Zhu et al. [Zhu 99] optimization techniques were used to determine the optimal locations and scheduling of dredging to minimize cost and obtain a channel where water depths are not less than some specified depth. The control of sedimentation can be extremely costly; hence optimization is a useful tool. The optimization problem is solved by the conjugate gradient method. The gradient of the cost function (objective function) is calculated by solving the adjoint problem. Through this study it was determined that for a nonlinear model the adjoint approach is only valid for small variations in dredging depth.

Mousavi and Ramamurthy [Mousavi 00] introduced a new composite algorithm for optimizing the operating policy of multi-reservoir systems. The model uses optimal control theory and penalty successive linear programming as techniques for modeling large and complex water supply systems. The objective function minimizes the required reservoir costs to supply specified yields. 
In their work, Cunha et al. [Cunha 99] use the Newton search method to solve the hydraulic network equations to obtain the least-cost design of a looped water distribution network using simulated annealing. Simulated annealing works well for large-scale optimization problems that are cast in discrete or combinatorial form. The optimal water distribution design problems discussed in the study contain discrete elements. To keep these designs close to reality they must be formulated as large size, nonlinear mixed integer models. Results show that this method can provide high quality solutions for network design problems compared to past studies.

Piasecki et al. [Piasecki 97] used adjoint sensitivity analysis to determine the control of contaminant releases in rivers. Two-dimensional finite-element models are used to determine the hydrodynamic and mass transport conditions. The sensitivities of the loading parameters are computed. This method reproduces almost identical "sensitivity functions" with repeated numerical solutions.

River and channel flood routing are modeled using the nonlinear Muskingum models in Mohan's work [Mohan 97]. Mohan used genetic algorithms to estimate the parameters of the Muskingum models. Comparisons of the performance of the genetic algorithm and other parameter estimation procedures were evaluated. The genetic algorithm estimates the parameters quickly and objectively and performed better or as well as other methods to which it was compared.

Sanchez et al. [Sanchez 98] discuss the use of neural networks as a means of reducing computing costs of coastal sewage systems. Neural network calculations are used to reduce calculation time for studying the influence of storm discharge on the bacteriological quality of bathing waters. Neural networks maintain an approximation level similar to numerical solutions. The study concludes that 
neural network with optimized functional link is suitable for design of the collector network of coastal sewage systems when storm discharges occur.

Burg [Burg 99] developed an optimization strategy for open-channel flow problems. In his work, optimization methods were analyzed to determine their applicability to various problems. Table 2.2 shows a comparison of the various optimization methods. The code used in this work, HIVEL2D, is a high-fidelity implicit code. From the table, the discrete sensitivity analysis is reported to be best for this type of problem, whereas the continuous sensitivity analysis is suitable for explicit high-fidelity problems. The inverse method and genetic algorithms are not appropriate for this type of problem. The adjoint variable formulation or the quasi-analytic method, described below, may be used to compute the design space gradients. Updating the design variable is accomplished by the method of steepest descent with a step size, the method of steepest descent with a linear search, the Gauss-Newton algorithm, or the BFGS method. The technique was applied to channel expansion, channel contraction, and embedded body problems.

The adjoint method was used to calculate the design space derivative of the objective function, $F(Q(\vec{\beta}), \chi(\vec{\beta}), \vec{\beta})$ where $\mathrm{Q}$ is the steady-state flow variables, $\chi$ is the discretized grid, and $\vec{\beta}$ is the vector of design variables or

$$
\frac{d F}{d \beta_{i}}=\frac{\partial F^{T}}{\partial Q} \frac{\partial Q}{\partial \beta_{i}}+\frac{\partial F^{T}}{\partial \chi} \frac{\partial \chi}{\partial \beta_{i}}+\frac{\partial F}{\partial \beta_{i}}
$$

In this equation $\frac{d F}{d \beta_{i}}$ is the total variation of $\mathrm{F}$ with respect to the design variable $\beta_{i} \cdot \frac{\partial F}{\partial Q}, \frac{\partial F}{\partial \chi}$, and $\frac{\partial F}{\partial \beta_{i}}$ can be calculated since the dependency of $\mathrm{F}$ on $\mathrm{Q}, \chi$, and $\vec{\beta}$ is explicit. $\frac{\partial \chi}{\partial \beta_{i}}$ can be estimated by finite differencing the results of the grid generation code or by differentiating the explicit dependencies between the grid and the design variables with hand-differentiation, complex Taylor series expansion, or ADIFOR 
Table 2.2: Comparison of Optimization Methods [Burg 99]

\begin{tabular}{|c|c|c|c|}
\hline $\begin{array}{c}\text { Optimization } \\
\text { Method }\end{array}$ & Type of & Advantages & Disadvantages \\
\hline \hline Inverse & Analytic & Highly & Not Generally \\
Methods & Formula & Efficient & Applicable \\
\hline Genetic & Discontinuous, & Avoids Local & Many \\
Algorithm & Discrete, & Minima, & Function \\
(Probabilistic & Cheap Simulations, & No Gradient & Evaluations \\
Methods) & Multi-Modal & Needed & \\
\hline Finite & Any & Easiest & Large Computer \\
Difference & & To Use & Cost, Accuracy \\
\hline ADIFOR, & Any & Highly Accurate & Large \\
CTSE & & Derivative, & Computational \\
& & Easy to Use & Cost \\
\hline Continuous & Explicit & Computationally & Derive and \\
Sensitivity & High-Fidelity & Efficient & Solve \\
Analysis & & Accurate & Jacobian \\
\hline Discrete & Implicit & Derivatives, & Matrix \\
Sensitivity & High-Fidelity & Efficient & Needed \\
Analysis & & &
\end{tabular}

[Bischof 92]. Estimation of the vector $\frac{\partial Q}{\partial \beta_{i}}$ with finite differences would require an additional steady-state simulation. This term, however, also appears in the derivative of the discretized system of governing equations, $W(Q(\vec{\beta}), \chi(\vec{\beta}), \vec{\beta})=0$ or

$$
0=\frac{d W}{d \beta_{i}}=\frac{\partial W}{\partial Q} \frac{\partial Q}{\partial \beta_{i}}+\frac{\partial W}{\partial \chi} \frac{\partial \chi}{\partial \beta_{i}}+\frac{\partial W}{\partial \beta_{i}}
$$


As in equation 2.1, all terms except for $\frac{\partial Q}{\partial \beta_{i}}$ can be calculated without the need for a steady-state simulation. Equation 2.2 and Equation 2.1 are used to estimate the design space derivative $\frac{d F}{d \beta_{i}}$.

For the adjoint variable formulation of discrete sensitivity analysis, equation (2.2) is multiplied by an adjoint vector $\lambda$ and added to equation (2.1). This yields

$$
\frac{d F}{d \beta_{i}}=\left(\frac{\partial F^{T}}{\partial Q}+\lambda^{T} \frac{\partial W}{\partial Q}\right) \frac{\partial Q}{\partial \beta_{i}}+\left(\frac{\partial F^{T}}{\partial \chi}+\lambda^{T} \frac{\partial W}{\partial \chi}\right) \frac{\partial \chi}{\partial \beta_{i}}+\frac{\partial F}{\partial \beta_{i}}+\lambda^{T} \frac{\partial W}{\partial \beta_{i}}
$$

To avoid having to solve for $\frac{\partial Q}{\partial \beta_{i}}$, the coefficient of $\frac{\partial Q}{\partial \beta_{i}}$ is set equal to zero. That is

$$
\begin{aligned}
& \frac{\partial F^{T}}{\partial Q}+\lambda^{T} \frac{\partial W}{\partial Q}=0 \\
& \frac{\partial W^{T}}{\partial Q} \quad \lambda=-\frac{\partial F}{\partial Q}
\end{aligned}
$$

$\lambda$ must only be calculated once for each objective function and is independent of the design variables for the adjoint variable formulation. The adjoint variable formulation scales with the number of functions and constraints and is appropriate for problems with only one objective function and a few constraints. The direct formulation scales with the number of design variables.

For the direct formulation of discrete sensitivity analysis, or quasi-analytic method, the vector $\frac{\partial Q}{\partial \beta_{i}}$ is obtained by solving equation (2.2) directly or

$$
\frac{\partial W}{\partial Q} \frac{\partial Q}{\partial \beta_{i}}=-\frac{\partial W}{\partial \chi} \frac{\partial \chi}{\partial \beta_{i}}-\frac{\partial W}{\partial \beta_{i}}=-\left.\frac{d W}{d \beta_{i}}\right|_{\text {Qfixed }}
$$

The vector $\frac{\partial Q}{\partial \beta_{i}}$ must be calculated for each design variable. This makes the computational cost of the direct formulation greater than that of the adjoint variable formulation when the number of design variables is greater than 
the number of objective function and constraints. However, when using the Gauss-Newton optimization algorithm the number of objective functions to be differentiated is much larger than the number of design variables since this algorithm views each term in the objective function as a separate residual function. For this reason, the quasi-analytic method is more cost effective. [Burg 99]

The work presented in this research deals with one and two design variable problems. The objective function is in the non-linear least squares form, so the Gauss-Newton method may be used. The quasi-analytic method is used to calculate the design space derivative. 


\section{CHAPTER III METHODOLOGY}

Burg's work was applied to several types of high-velocity channel transitions. He did not address issues such as the confluence of two channels, channels with multiple design flows, or channels with multiple transition. The purpose of this research is to expand Burg's tests to include those issues not addressed by Burg by applying his work to a "real-world" problem. The Walnut Creek Flood-Control Channels are high-velocity channels that include hydraulic structures such as contractions, bends, and a confluence. The design flows from the two channels differ by as much as 8,400 cfs, and the channels contain both sub- and supercritical flows with a hydraulic jump. The Walnut Creek Flood-Control Project has had much attention and required substantial re-design. As with most urban floodcontrol channels, increased population and urbanization generally necessitates a re-evaluation of the design.

This research can be broken into two stages as illustrated in Figure 3.1. The

first stage deals with the preliminary issues such as numerical representation of the problem, determination of the design criterion or objective function, and determination of the design variables and ranges via parameter sensitivity analysis. The second stage deals with the actual optimization of the problem. There must be a process by which the changes to the design variables are incorporated into the model. Once the model is updated, the HIVEL2D code is used to simulate the 
flow for the new design. The objective function is evaluated once the steady-state solution is reached, and the design space derivatives are computed. This process is repeated for a prescribed number of iterations until the problem has converged to an answer. Each step of this process is discussed in detail in the following sections.

\subsection{Numerical Representation of Physical Problem}

Representation of the physical problem for numerical analysis is the most important part of numerical modeling. It is crucial that all areas vital to the behavior of the system are well represented, thus more sophisticated grids are required.

The first step in numerical modeling is to define the basic geometry of the problem. This can be done by locating important points in the design such as the beginning of a contraction or expansion. Once these points are identified, the grid can be generated. There are a number of processors for generating grids. The one used for this work is the Surface-Water Modeling System or SMS. SMS is a preand post-processor for building grids and viewing solutions [SMS 00].

Once the problem geometry is represented and the grid is generated, the boundary and initial conditions can be specified. At this point the problem is ready for code application to solve for the flow variables. The steady-state solutions can then be examined with a post-processing technique. Using the results from the flow simulation, a grid convergence test is conducted. This determines the appropriate grid refinement required to adequately represent the problem. 


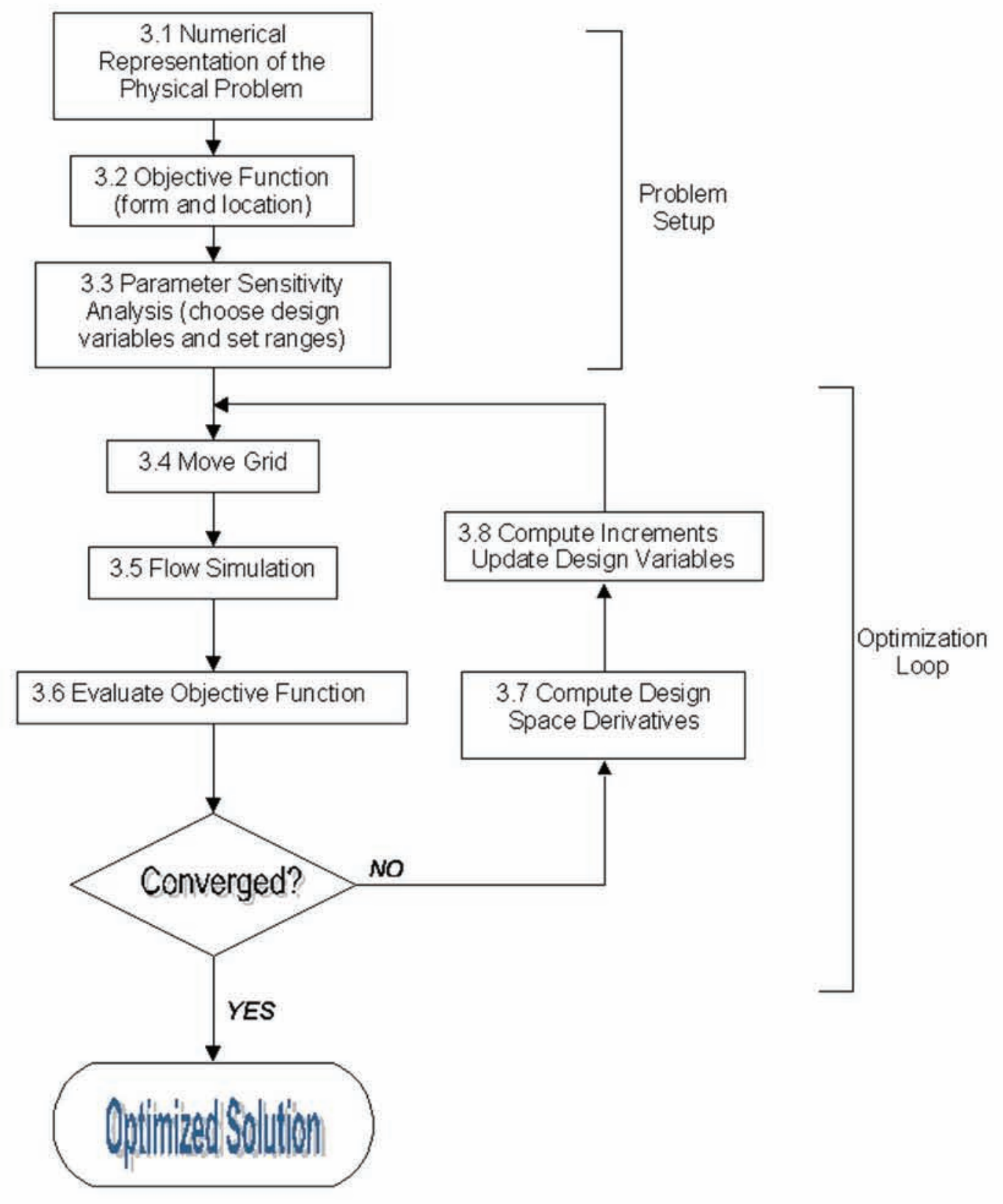

Figure 3.1: Flow Chart of Method for Optimizing High-Velocity Channel 


\subsection{Objective Function}

A variety of objectives can be defined for high-velocity channels. The objective functions can measure variation in depth to produce near uniform flow depths, depth raised to some power, and/or energy loss through a transition. The functions can be functions of the flow variables, the grid, and/or the design variables. A major concern for engineers designing high-velocity channels is water overtopping the walls and reaching the bridges. To avoid this, the water depth and cross-waves or sloshing should be minimized. The objective function used by Burg is given by

$$
F(\vec{\beta})=\sum_{\vec{x}_{k} \in \Omega_{F}}\left(h_{a v e}(\vec{\beta})-h_{k}(\vec{\beta})\right)^{2}
$$

where $\mathrm{h}$ is the depth and $h_{\text {ave }}$ is the average depth over the entire area of interest[Burg 99]. Equation 3.1 measures the non-uniformity of the flow depths by measuring the variation of the depths from the average depth over a given area. The function is explicitly dependant only on $\mathrm{Q}$ and is a continuous function; otherwise the design space derivatives would not exist. A disadvantage of this function is its inability to detect subcritical flow. If flow becomes subcritical, the water surface is smooth thus satisfying the equation, but the depth is substantially increased.

Another possible objective function is given by

$$
F(\vec{\beta})=\sum_{\vec{x}_{k} \in \Omega_{F}}\left(h_{k}\right)^{n}
$$

where n equals the power. Equation 3.2 is the sum of the depth raised to a power. This function detects increased depths such as would occur for subcritical flows. This equation is not as sensitive to roughness in the water surface as Equation 3.1. 


\subsection{Parameter Sensitivity Analysis}

Several model parameters exist for a high-velocity channel. To determine the parameters that have the most effect on the model globally and locally, a parameter

sensitivity analysis is performed. Parameters are adjusted independently, and the resulting objective function values are recorded. The parameters are adjusted to ensure a wide range of possible values is considered. The parameters that have the greatest effect on the objective function are determined. Those parameters that have little or no effect are not considered in the optimization process. The parameters that point to an obvious solution or trend through the sensitivity analysis are set according to the analysis and are not optimized. The resulting objective function values are also used to determine the parameters that can be optimized locally and the parameters that have a global effect on the model.

\subsection{Moving the Grid}

When a new design is produced by the optimization code the new grid will need to be developed to describe the new design. A system for moving the mesh for the parameters must be developed. Moving or re-generating the grid is a very important part of the process. Most of an engineer's time is devoted to generating an accurate representation of the area of interest, and changes to the mesh must have the same attention. Three ways of re-generating a grid for optimization are integration of an existing grid generation code within the optimization code, develop and encode a set of rules based on the design variables and grid parameters, and modify an existing grid based on the design variables.

Most grid generation tools allow users to save steps taken to generate the grid. These steps can be put into a script for easy re-generation of the grid. 
This technique, however, requires the grid to be re-generated for every design variable. For small problems, re-generation of the entire grid for every design variable would not be very expensive. The re-generation of the entire grid would be quite computationally expensive for more sophisticated problems of larger scale.

Another method of generating the new grid is by developing and encoding a set of rules based on the design variables and grid parameters. This method allows for many design variables and grid parameters to control the shape of the boundary and the grid spacing. Once the boundary is determined, a grid is generated. Burg [Burg 99] uses this method to generate structured, multi-block grids.

The final method, which is the method used in this work, modifies an existing grid based on the design variables. Due to the fact that the problems used in this study contain several hydraulic structures, a structured mesh is not appropriate. The mesh used is an unstructured, two-dimensional mesh. Boundary perturbations are established for the design variables using the optimization procedure. Once the boundaries have been moved, a call is made to a Laplacian solver to determine the displacement in the $\mathrm{x}$ and $\mathrm{y}$ directions of the interior nodes for the radius of curvature problem or in the z-value for the super elevation problem [Carey 99]. These displacements are then used to calculate the new coordinates for the nodes in the interior of the domain. The equations being solved are given by Equation 3.3. This technique is used to move the curve for the various radius of curvature values and is illustrated in Figure 3.2. This technique pushes toward a uniform mesh spacing while honoring the boundary conditions for the radius of curvature problem, and linearly adjusts the z-values for the super elevation problem.

$$
\nabla^{2} x_{d}=0, \nabla^{2} y_{d}=0, \nabla^{2} z_{d}=0
$$




\subsection{Flow Simulation}

The flow analysis is performed using HIVEL2D. HIVEL2D is a finite element model that solves the two-dimensional shallow water equations. The results are a steady-state representation of the flow. Details of HIVEL2D are given in the previous chapter.

\subsection{Termination Criteria}

The optimization terminates when the termination criteria is met. For this study, the optimization criteria is given by Equation 3.4. When the change in the objective function value is less than $10^{-7}$ the code terminates.

$$
F\left(\beta_{n}\right)-F\left(\beta_{n-1}\right)<10^{-7}
$$

\subsection{Function Evaluation}

Once the steady-state solution is obtained, the objective function is evaluated. The selected objective functions are expressed in the non-linear least squares form, thus the Gauss-Newton optimization algorithm can be used. That is, the objective function is such that the sum of the squares of the deviation from the desired answer is minimized. The Gauss-Newton method takes advantage of the structure of the objective function to approximate the Hessian. Results for objective function values using Equation 3.1 and Equation 3.2 are compared. Generally, the equations give similar results. Either equation can be used and similar results obtained. The objective function used in this study is given in Equation 3.1. 

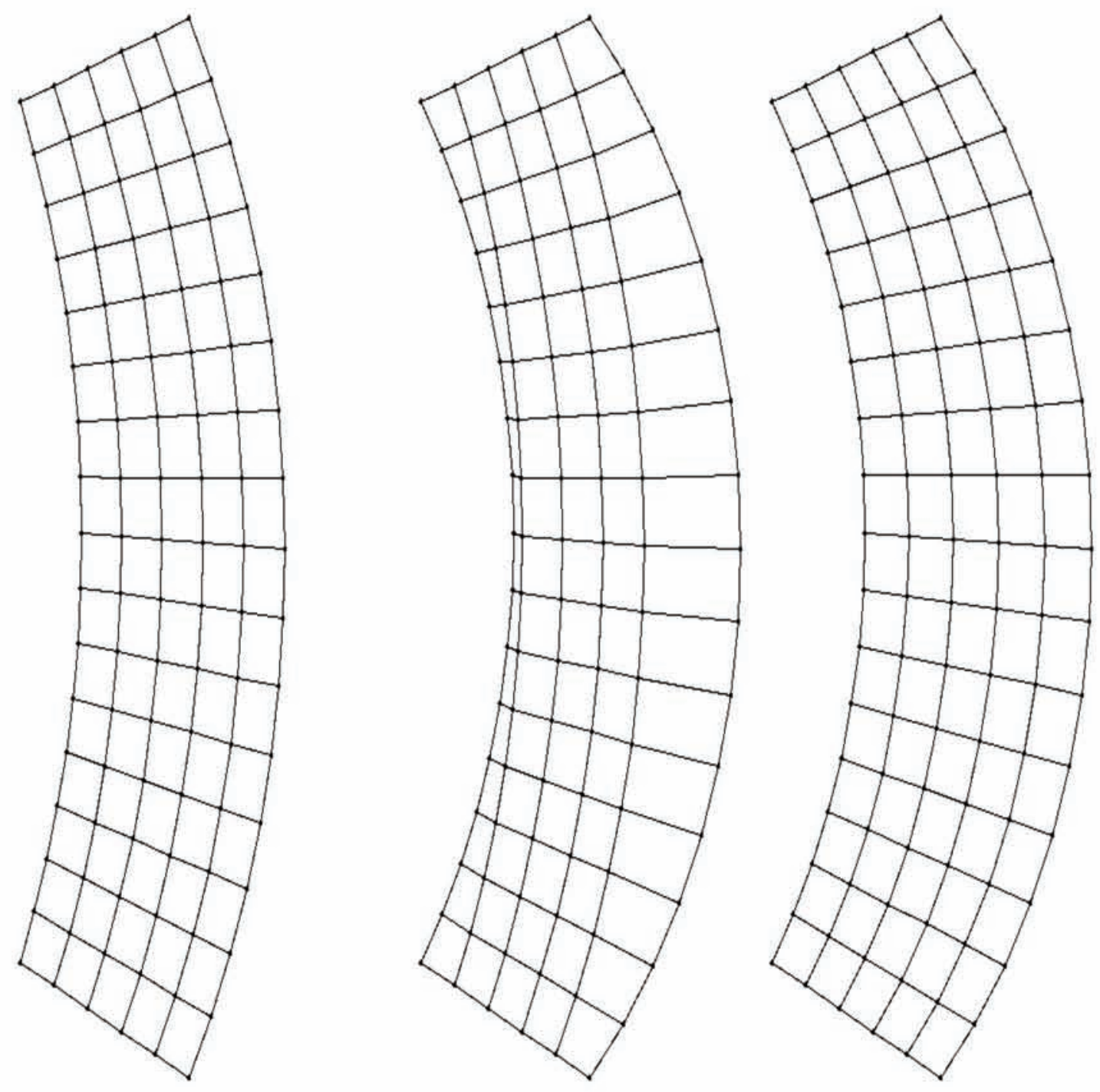

Figure 3.2: Laplacian Smoothing Technique 


\subsection{Design Space Gradient Calculation}

For calculating the design space gradient, the fewer steady-state solution calculations required the better. This is due to the fact that complex problems, as the one in this study, contain many hydraulic features that make steady-state solution calculations computationally expensive. Sensitivity analysis does not require any additional steady-state solutions for the types of problems addressed in this work and thus is quite suitable for the problems discussed in this work. The discrete sensitivity analysis requires that the objective function be evaluated only at steady state; therefore the gradient estimation routines are only needed once steady-state flow has been reached. The code used for the optimization process gives the user the option of using the adjoint variable formulation or the quasianalytic method to calculate the derivatives. The adjoint variable formulation is efficient computationally when the number of design variables is larger than the number of objective functions. For this study, the goal is to minimize Equation 3.1. This equation is in the general non-linear least squares form illustrated by Equation 3.5.

$$
F(\vec{\beta})=\sum_{k=1}^{N} f_{k}^{2}(\vec{\beta})
$$

$f_{k}(\vec{\beta})$ is the residual function. For Equation 3.1, $f_{k}(\vec{\beta})=h_{\text {ave }}-h$. In this application, there is one residual defined at each of the 41 nodes illustrated in Figure 3.3. The Gauss-Newton method is applied to minimize each residual with respect to the selected design variable. Hence, for the problems in this research there are 41 constraint equations and one or two design variables. Consequently, the quasi-analytic formulation would be the most efficient method of calculating 
the necessary design space gradients. Using this formulation enables calculation of the design space gradients by solving one additional linear system for each design variable for each steady-state HIVEL2D simulation. [Burg 99]

\subsection{Design Variable Update}

For updating the design variables, Burg's code gives the option of using the method steepest descent with a step-size, the method of steepest descent with a linear search, the Gauss-Newton Algorithm, or the BFGS updating method [Burg 99]. The methods used in this research are the method of steepest descent with a step-size, the method of steepest descent with a linear search, and the Gauss-Newton Algorithm. 


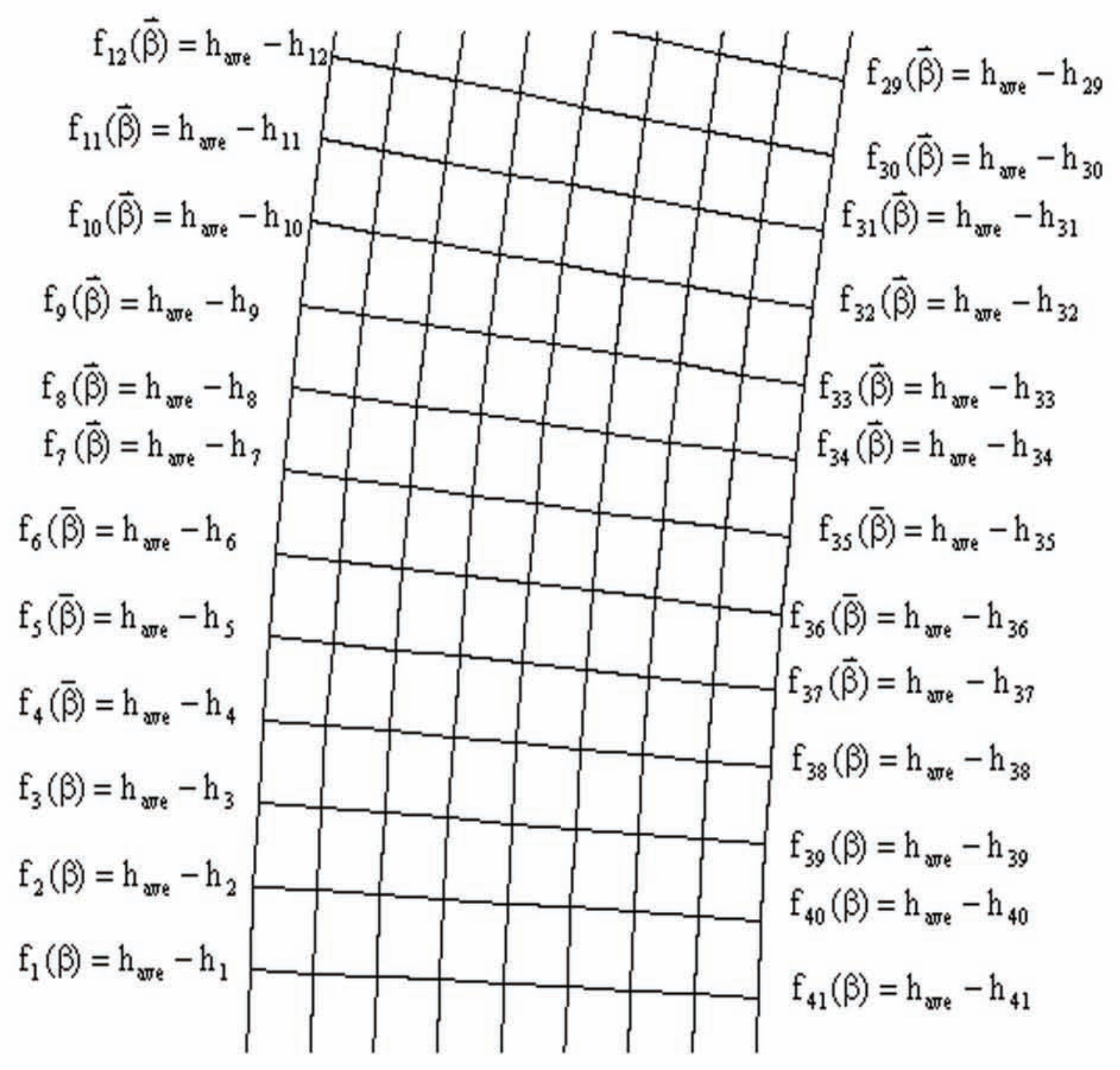

Figure 3.3: Residuals at Each Node in the Objective Space 


\section{CHAPTER IV}

\section{RESULTS}

The problem at Walnut Creek was initially addressed using a physical model. Engineers using the existing channel and the engineering design manual recommendations developed an initial design. The design was then used to build a physical model of the site. Once the physical model was completed, the problem areas and potential problem areas were noted and modifications were designed. Adding some features, such as the divider wall, can be inexpensive. To test various lengths or orientations of a divider wall, sections of varying lengths are constructed that can be held into place and adjusted for orientation by engineers until the flow conditions improve. Adjusting curves and channel widths would require removing parts of the model and rebuilding them with the new design. For this reason, the physical model study was able to test more options for the divider wall lengths and orientation than for the other design parameters.

The first step to apply Burg's optimization code to the Walnut Creek study is to represent the site numerically. The study area begins at the junction of Walnut Creek and the San Ramon Bypass Channel and extends upstream in the San Ramon Bypass Channel approximately 180 feet as indicated in Figure 4.1. Through parameter sensitivity analysis discussed later in this chapter, the super elevation and radius of curvature in the San Ramon Bypass Channel (Figure 4.2) are selected as the system design variables for this demonstration. The choice of an objective 
function is a major part of the study. For an optimization problem, the objective function must contain the engineering judgment. All of the necessary requirements for the channel, such as smooth flow and minimal depth, must be included in the objective function. Using the objective function, a parameter sensitivity analysis is conducted to determine the design parameters that have an affect on the model. It must also be determined whether design variables have global or local effects. The sensitivity analysis reduces the number of design variables to only those to which the model is sensitive. Once the design parameters are determined, Burg's code is applied to find an improved design. The design parameters for this study are the super elevation and radius of curvature of the curve in the San Ramon Bypass Channel. The objective is to minimize overtopping of the model walls and water surface roughness just upstream of the confluence. The super elevation, denoted by $\Delta y$ in Figure 4.3 , and radius of curvature, denoted by $\mathrm{R}$ in Figure 4.4 , of the curve in the San Ramon Bypass Channel are optimized separately.

\subsection{Numerical Representation of Physical Problem}

The first step in generating the mesh for the Walnut Creek study is to identify important points in AutoCAD. Figure 4.5 illustrates the identification of important points using AutoCAD. These points are then read into SMS [SMS 00]. The points are assigned a bed elevation value or z-value and used to generate a number of nodes via node interpolation using linear and arc interpolations. Once the nodes have been created the triangulation process is performed. Once the mesh is generated, the boundary conditions and initial conditions for the Walnut Creek study are applied. 


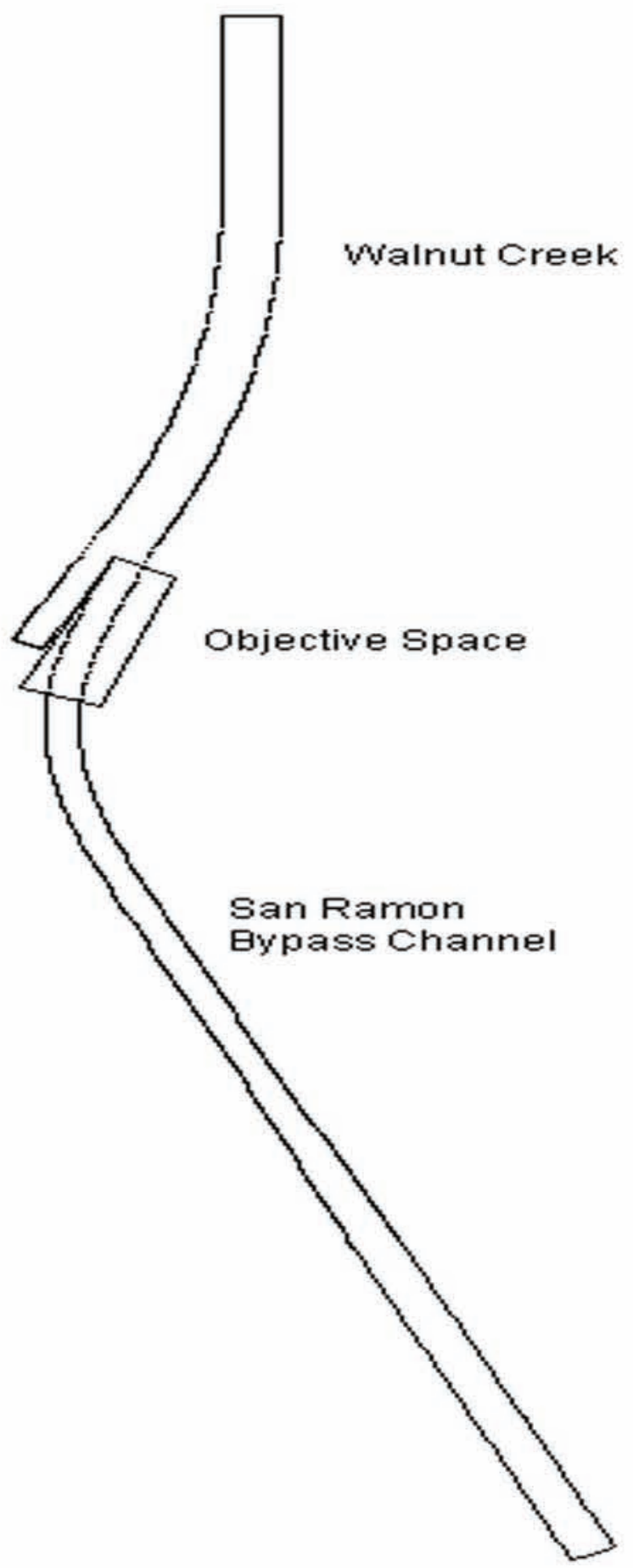

Figure 4.1: The Location of the Study Area. 


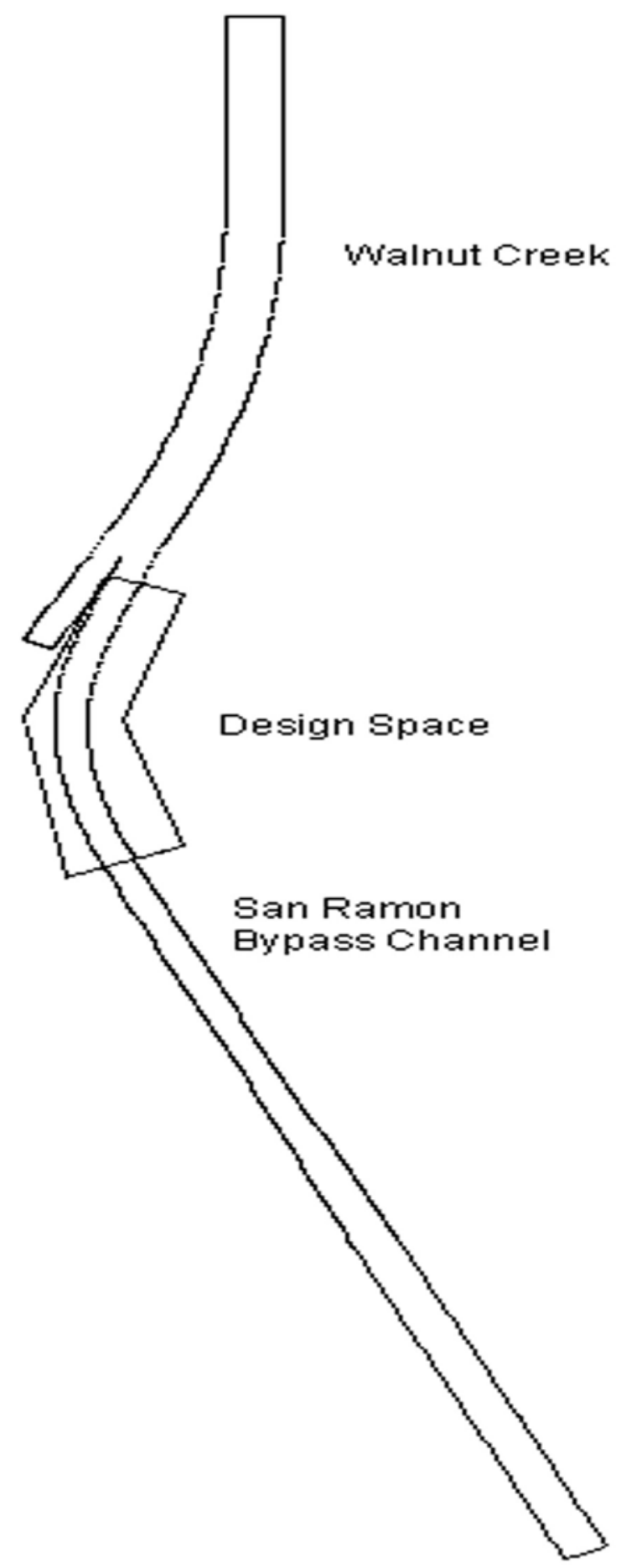

Figure 4.2: The Design Space for the Optimization Problems. 


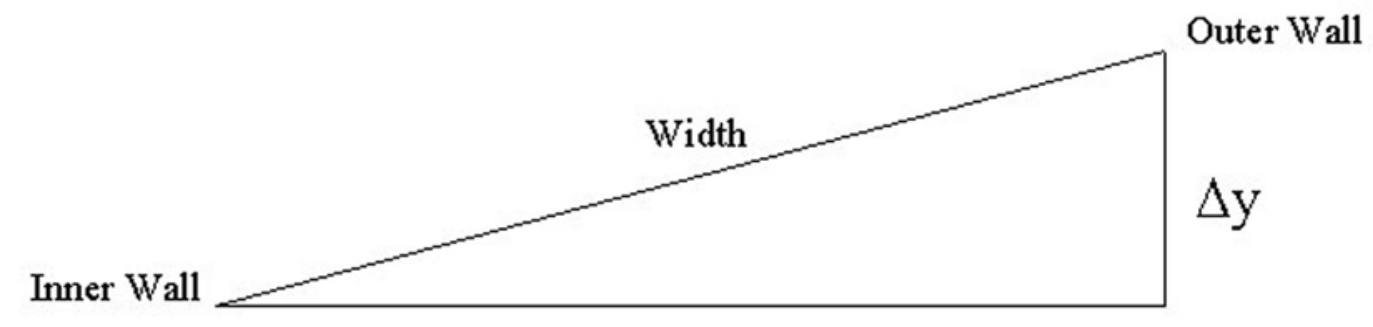

Figure 4.3: The Super Elevation in the Curve.

The Walnut Creek problem is very complex and includes a number of hydraulic phenomena. For this reason, the steady-state solution is more difficult to obtain. In order to prevent introducing artificial hydraulic jumps that are hard if not impossible for the model to push out of the system, a subcritical tailwater boundary was set to maintain a reasonable depth throughout the model. Once the jump was pushed through the model to the outflow boundary, the tailwater boundary was removed and the outflow was set to supercritical.

The appropriate grid resolution for the problem is determined via a grid convergence test. This is accomplished by generating an initial mesh with minimal resolution and increasing the resolution until the changes in the resulting solutions are minimal. For this study, the areas with solutions differing by more than $10^{-3}$ were refined. The resulting mesh consists of 4,592 elements and 5,016 nodes. This mesh is used for the optimization problems. 


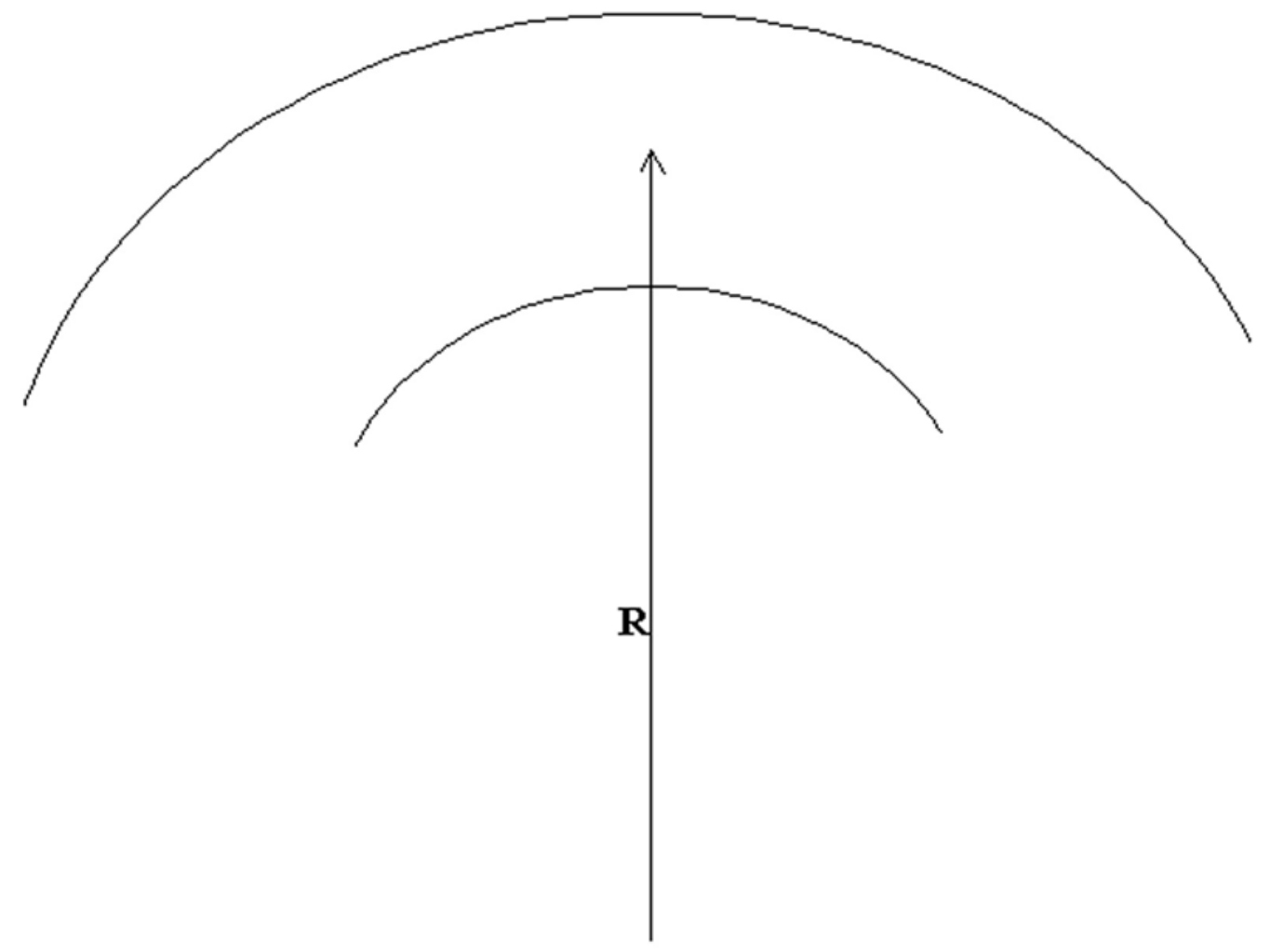

Figure 4.4: The Radius of Curvature for the Curve. 


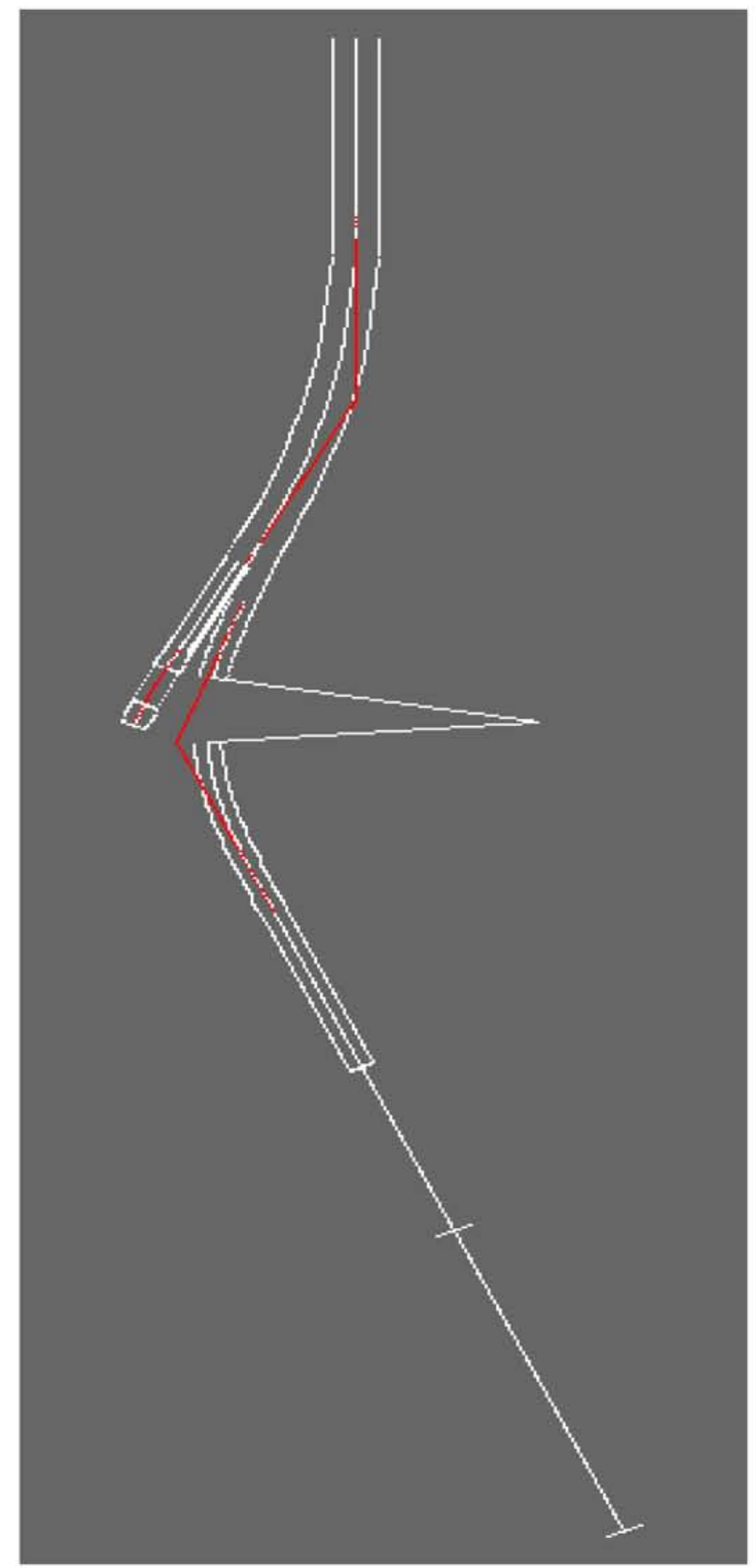

Figure 4.5: AutoCAD Depiction of Location used to Generate the Mesh. 
Once the mesh is created, boundary conditions are specified. The Walnut Creek problem consists of three boundary conditions. Inflow boundaries are set for Walnut Creek and San Ramon Bypass Channel. The inflow for Walnut Creek is supercritical with a depth of 11.83 feet and a rate of 6,800 cfs. For San Ramon Bypass Channel the inflow is supercritical at a depth of 15 feet and rate of 15,200 cfs. These values represent a 100-year frequency event. An outflow boundary in Walnut Creek is set to supercritical flow. Figure 4.6 illustrates the mesh and boundary conditions. The Manning's n value for the entire mesh is 0.014 .

The numerically defined problem is run to steady-state using HIVEL2D. The solution is compared to plots given in the physical model report [Davis 87]. The comparisons indicate that good computational fluid dynamics analysis is achieved. Figure 4.7 shows a qualitative comparison of he resulting water surface elevations of the physical model and the numerical model.

\subsection{Objective Function}

The Walnut Creek physical model study considered three major areas when determining an appropriate design. The areas are located in the circular curve section of Walnut Creek downstream of the confluence, in the section of Walnut Creek located just upstream of the confluence, and in the curved section of the San Ramon Bypass Channel just upstream of the confluence. However, since flow in the San Ramon Bypass Channel is supercritical everywhere, it is only affected by changes made upstream of the area. For this reason, we will only consider the first two areas to determine what has an affect on the overall model. These areas will be referred to as area 1 and area 2, respectively, and are shown in Figure 4.8. The concern in the areas is with the water overtopping the walls. This can 


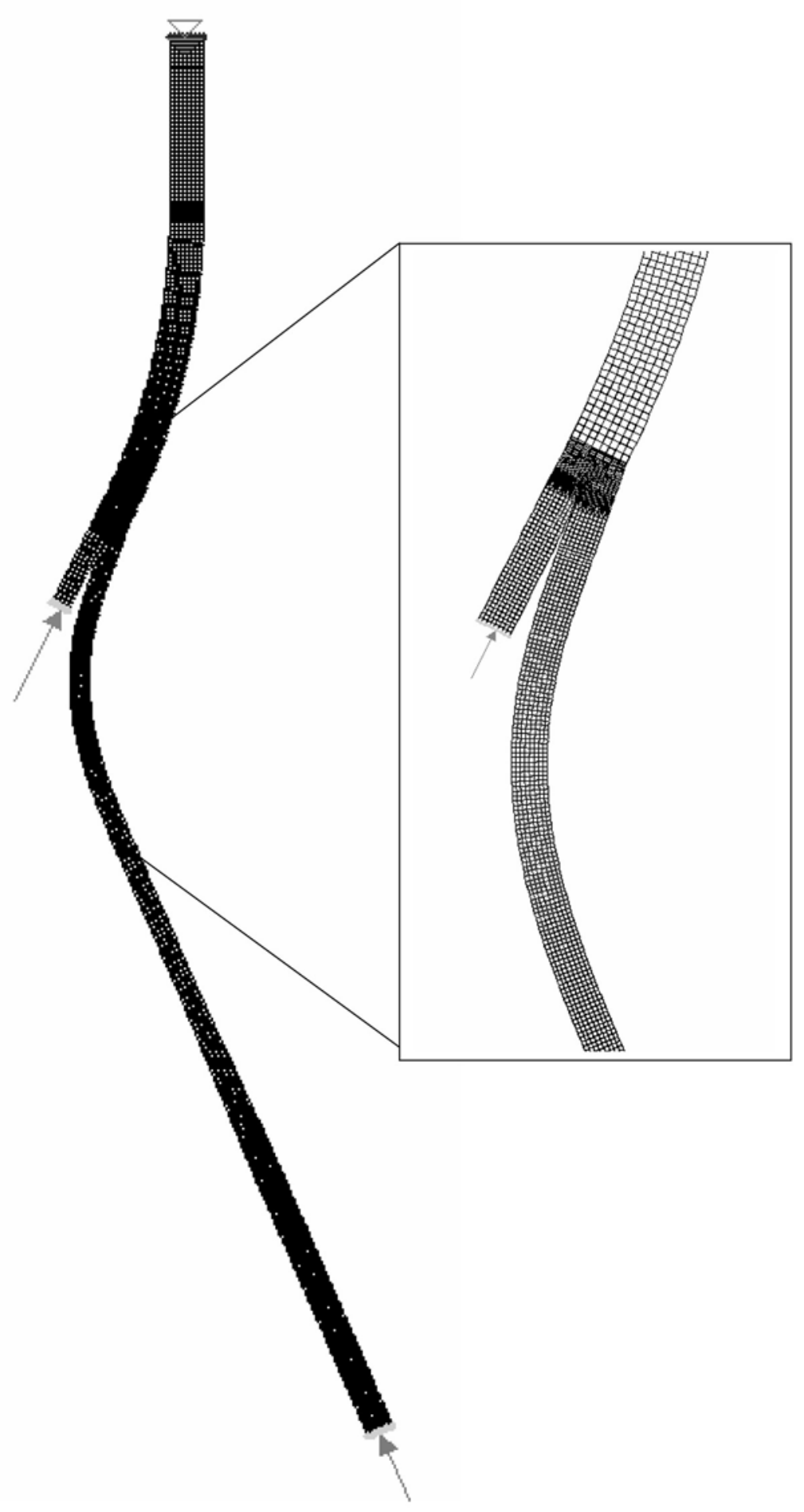

Figure 4.6: The Finite Element Representation and Boundary Conditions. 

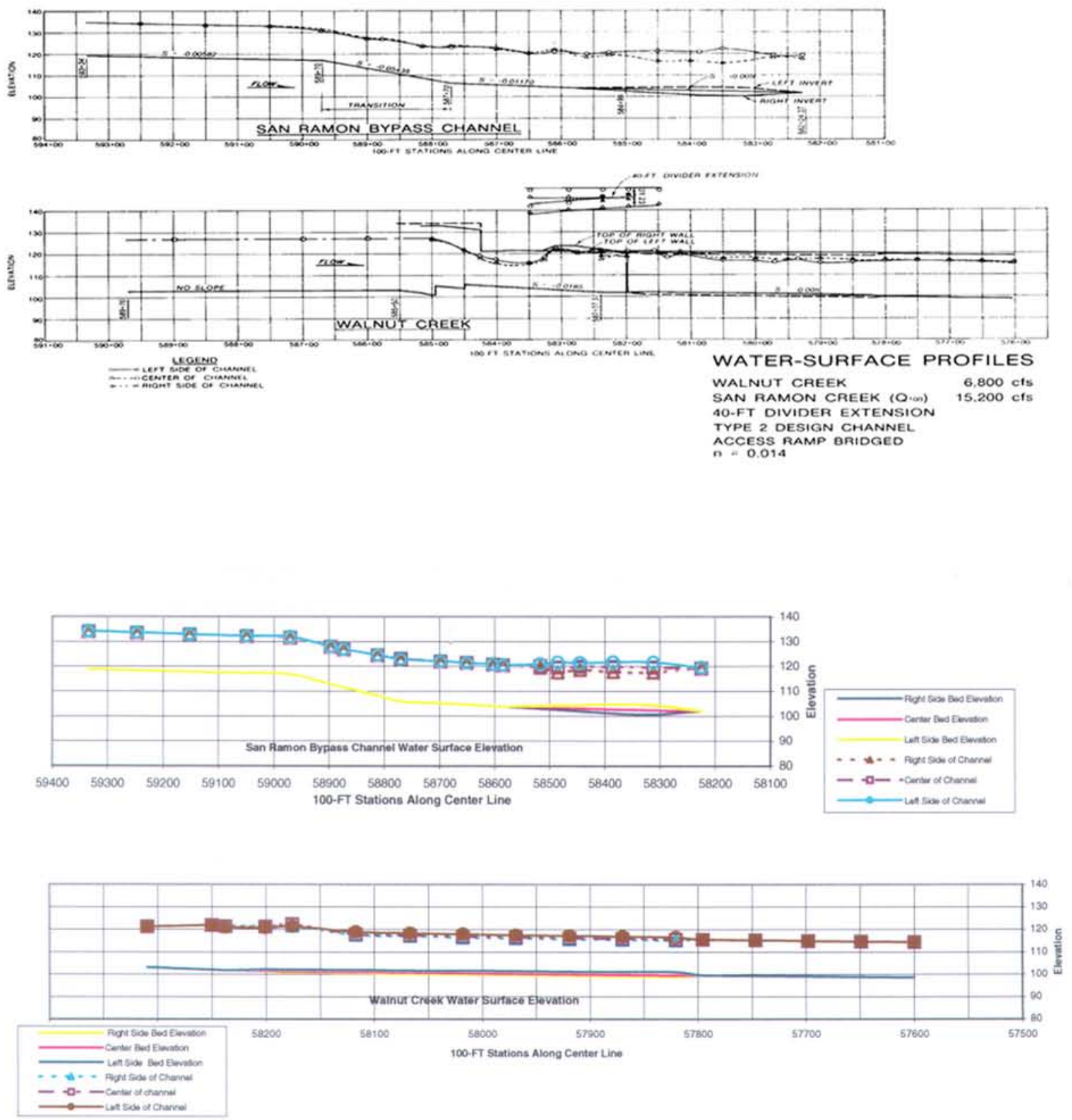

Figure 4.7: Water Surface Elevations for the Physical and the Numerical Models. 
be avoided by decreasing the overall depth and/or by decreasing the roughness of the water surface. Both Equation 3.1 and Equation 3.2 are used to perform the parameter sensitivity analysis. The parameter sensitivity analysis discussed later in this chapter indicate that both Equation 3.1 and Equation 3.2 give the same results. Further tests are conducted with the super elevation and radius of curvature problems, which also indicate that either equation could be used and results would be the same. For this study, Equation 3.1 is chosen as the objective function for the optimization problems.

\subsection{Parameter Sensitivity Analysis}

In a practical case, such as this, there are potentially many design parameters. The optimization should only be performed on the design parameters that have a significant impact on the model. A parameter sensitivity analysis is used to determine the design variables to which the model is most sensitive. The primary objectives of the Walnut Creek physical model study were to determine the adequacy of the San Ramon Bypass Channel and the Walnut Creek-San Ramon junction and to develop modifications to improve the adequacy. The major areas considered were the length of a divider wall extension located at the junction, the width of the San Ramon Bypass Channel, and the super elevation and radius of curvature of the curve located just upstream of the junction. A suite of test cases is run to assess the sensitivity of the model to the various parameters. The list of runs made to test the model sensitivity are given in Table 4.1. Both objective functions were evaluated for each test case in area 1 and area 2.

One parameter explored is the super elevation in the curve upstream of the confluence. The original super elevation is 3.82 feet. Three other super elevation 


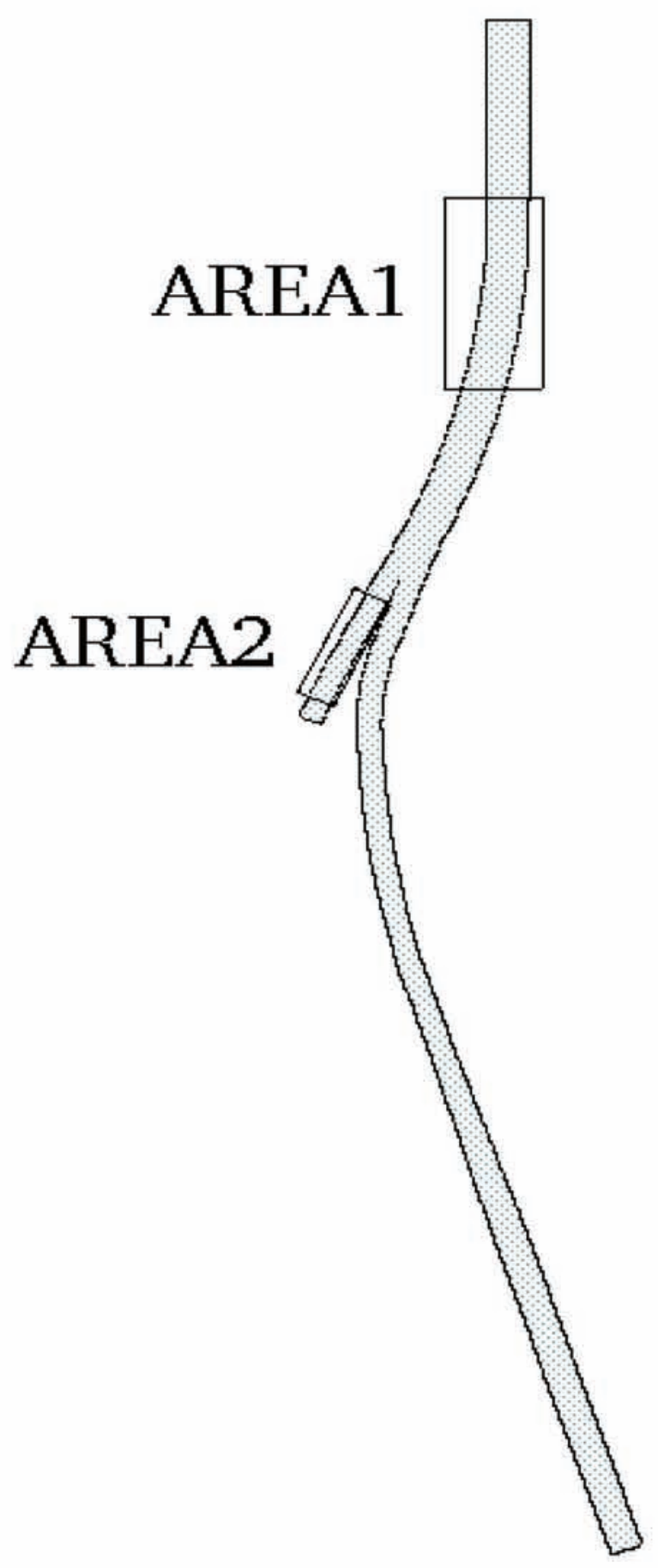

Figure 4.8: Objective Space for Optimization. 
Table 4.1: Parameter Sensitivity Runs

\begin{tabular}{|c|c|c|c|c|c|c|}
\hline Name & $\begin{array}{c}\text { Width of } \\
\text { San Ramon } \\
\text { Channel (ft) }\end{array}$ & $\begin{array}{c}\text { Super } \\
\text { Elevation (ft) }\end{array}$ & $\begin{array}{c}\text { Entrance } \\
\text { Spiral } \\
\text { Length (ft) }\end{array}$ & $\begin{array}{c}\text { Exit } \\
\text { Spiral } \\
\text { Length (ft) }\end{array}$ & $\begin{array}{c}\text { Radius of } \\
\text { Curve(ft) }\end{array}$ & $\begin{array}{c}\text { Divider } \\
\text { Wall } \\
\text { Length (ft) }\end{array}$ \\
\hline \hline Finaldes & 23 & 3.82 & 200 & 87.5 & 285 & 40 \\
\hline Se0 & 23 & 0 & 200 & 87.5 & 285 & 40 \\
\hline Se2 & 23 & 2 & 200 & 87.5 & 285 & 40 \\
\hline Se6 & 23 & 6 & 200 & 87.5 & 285 & 40 \\
\hline Width20 & 20 & 3.82 & 200 & 87.5 & 285 & 40 \\
\hline Width25 & 25 & 3.82 & 200 & 87.5 & 285 & 40 \\
\hline R350 & 23 & 3.82 & 135.45 & 49.72 & 350 & 40 \\
\hline R400 & 23 & 3.82 & 97.04 & 9.997 & 400 & 40 \\
\hline Dw10 & 23 & 3.82 & 200 & 87.5 & 400 & 10 \\
\hline Dw30 & 23 & 3.82 & 200 & 87.5 & 400 & 30 \\
\hline Dw64 & 23 & 3.82 & 200 & 87.5 & 400 & 63.8 \\
\hline Crc125 & 23 & 3.82 & 0 & 0 & 125 & 40 \\
\hline Crc250 & 23 & 3.82 & 0 & 0 & 250 & 40 \\
\hline Crc375 & 23 & 3.82 & 0 & 0 & 375 & 40 \\
\hline
\end{tabular}


values are also analyzed. The other values analyzed are no super elevation, 2 feet of super elevation, and 6 feet of super elevation. Figure 4.9 shows the bed elevations of the curve.

The resulting objective function values are given in Table 4.2. The results indicate that the model is not very sensitive to changes to the super elevation in the curve in area 1 and area 2. Figure 4.10 shows the water surface elevations for the original design and the three test cases for area 1 and area 2. Analyzing the results further show that there is minimal change in the water surface elevations for each case. The resulting depths are given in Figure 4.11. The water surface elevation values are differenced for each of the three cases. The resulting differences for the water surface elevations of the case with no super elevation and the case with a super elevation value of 2 feet indicate differences of approximately 0.15 feet occurring in a few locations just downstream of the curve. Examining the differences in the water surface elevations for the case with no super elevation and a super elevation value of 6 feet indicate differences of approximately 0.5 feet located in a few locations just downstream of the bend. Finally, the water surface elevations of the case with 2 feet of super elevation and 6 feet of super elevation are compared. Differences are visible in a few locations just downstream of the bend with values of approximately 0.25 feet. Super elevation is used to suppress the disturbances caused by curved transitions, which arise in the bend and persist downstream. This is exactly what is seen from the cases involving different super elevation values. The affects of changing the super elevation appear in the downstream end of the bend and just downstream of the bend. Area 1 and area 2 are not affected by these changes. This indicates that the super elevation in the curve can be optimized without considering the impact on area 1 and area 2 . In order to accurately optimize for super elevation, an objective function should 

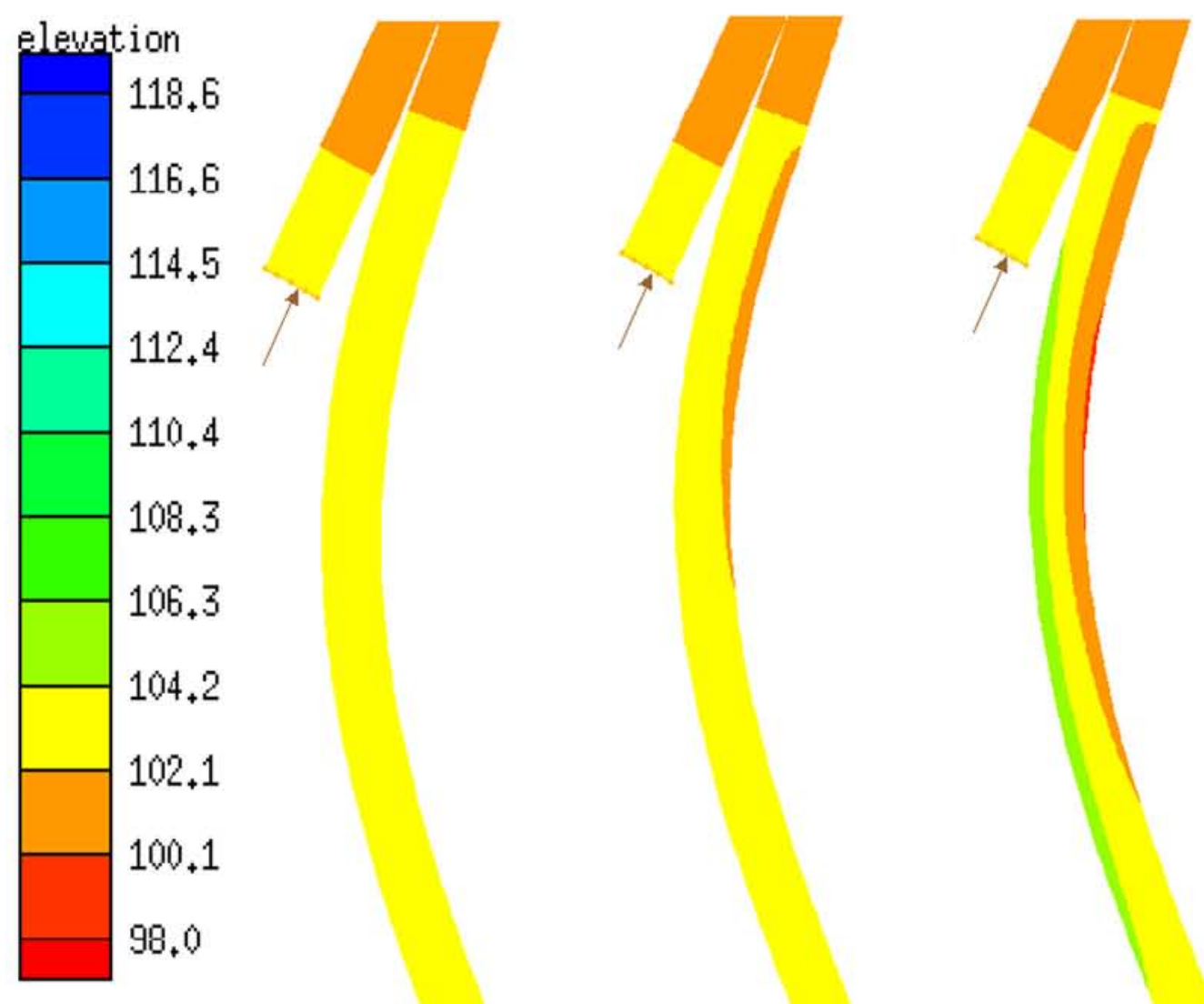

Figure 4.9: Bed Elevations for No(left), 2' (center), and 6' (right) Super Elevation. 
be located in the area impacted by changes to the super elevation. In this case that area is the downstream end of the bend and just downstream of the bend.

Table 4.2: Super Elevation Parameter Sensitivity Objective Function Values

\begin{tabular}{|c|c|c|c|c|}
\hline \multirow{2}{*}{ Run Name } & \multicolumn{2}{|c|}{ Objective Function 1 } & \multicolumn{2}{c|}{ Objective Function 2 } \\
\cline { 2 - 5 } & Area1 & Area2 & Area1 & Area2 \\
\hline \hline Se0 & 1.140224 & 1.519831 & 12589.39 & 5344.084 \\
\hline Se2 & 1.141895 & 1.528477 & 12589.71 & 5349.77 \\
\hline Se6 & 1.14185 & 1.637631 & 12597.34 & 5357.59 \\
\hline
\end{tabular}

The radius of curvature in this curve is also adjusted. The curve is restricted to a section of the channel that would not require additional changes to the design. That is, given two fixed channels design a curved transition including entrance and exit spirals to join the two. This restraint limits the radius of curvature to values between 285 feet and 410 feet. The initial radius of curvature is 285 feet. The lengths of the entrance and exit spirals are 200 feet and 87.5 feet, respectively. The different values of the radius of curvature tested were a radius of curvature of 350 feet with corresponding entrance and exit spiral lengths of 135.45 feet and 49.72 feet, respectively, and a radius of curvature of 400 feet with corresponding entrance and exit spiral lengths of 97.04 feet and 9.997 feet, respectively. The layout of the channels including bed elevations are depicted in Figure 4.12.

The steady-state solutions for the different scenarios indicate that area 1 and area 2 are not affected by changes in the radius of curvature. This is shown by the objective functions given in Table 4.3. The water surface profiles in area 1 and area 2 of the radius of curvature test cases, illustrated in Figure 4.13, shows that the 
Depth in Area1
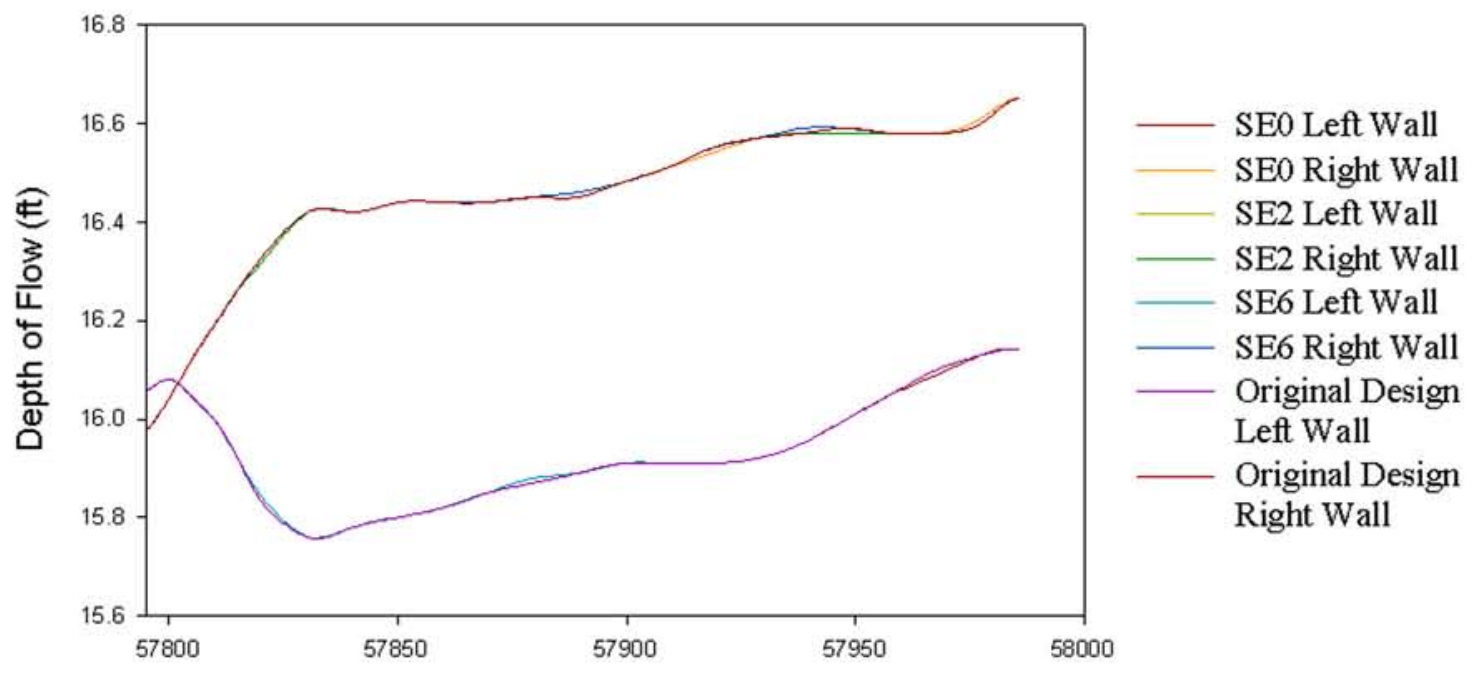

Distance Along Channel (ft)

Depth in Area2

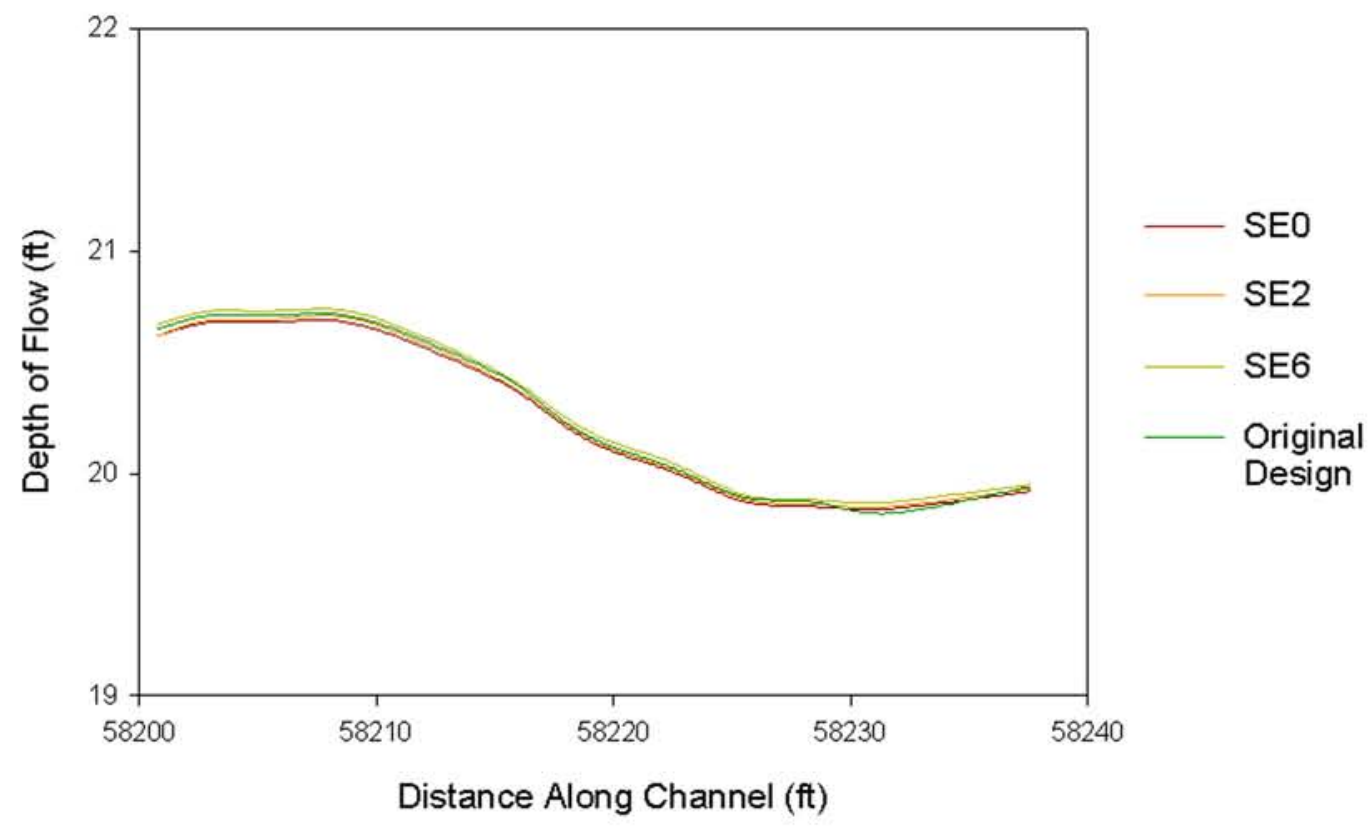

Figure 4.10: Depth Profile in Area1 and Area2 for Super Elevation Test Cases. 

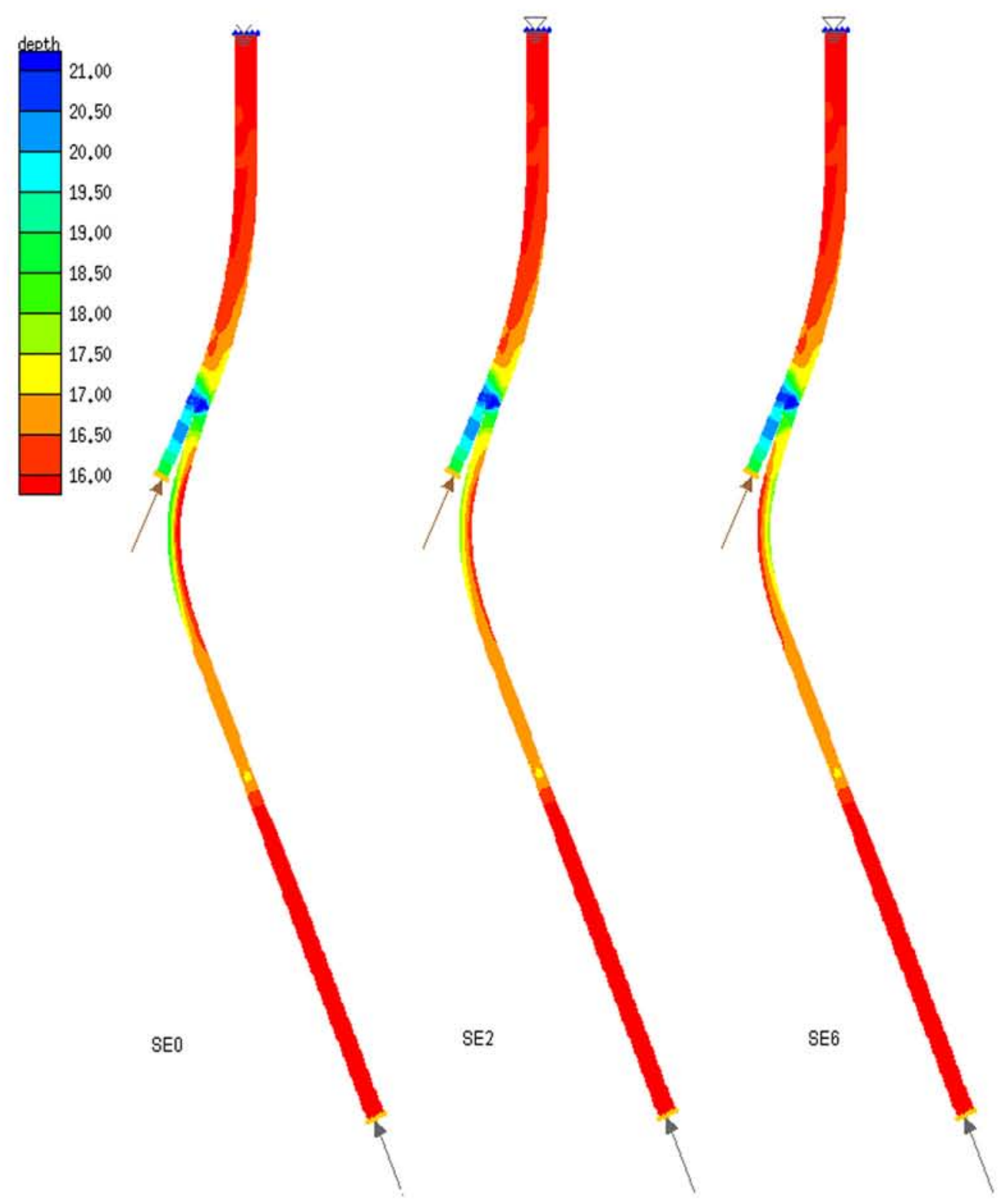

Figure 4.11: Resulting Depths for the Super Elevation Test Cases. 
changes in the surface water elevations is negligible for each change in the radius of curvature. The impact of the changes to the curved transition appear in the curve and just downstream of the curve. This can be optimized for local concerns without regard to the area 1 and area 2 impacts. For this study, the objective function is located within the bend and just downstream of the bend to optimize the radius of curvature.
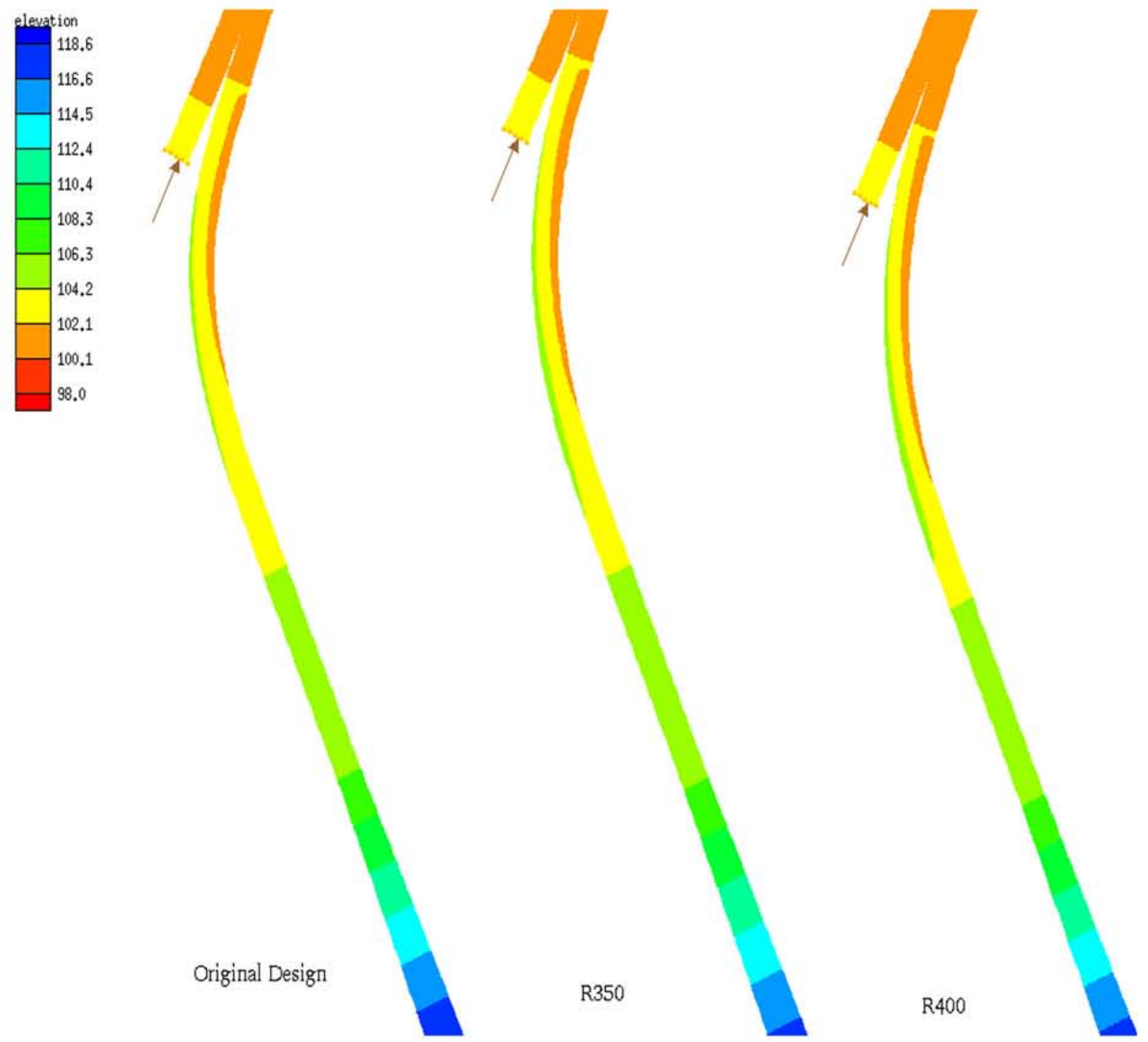

Figure 4.12: Bed Elevations of the Original Curve, 350' Radius, and 400' Radius. 

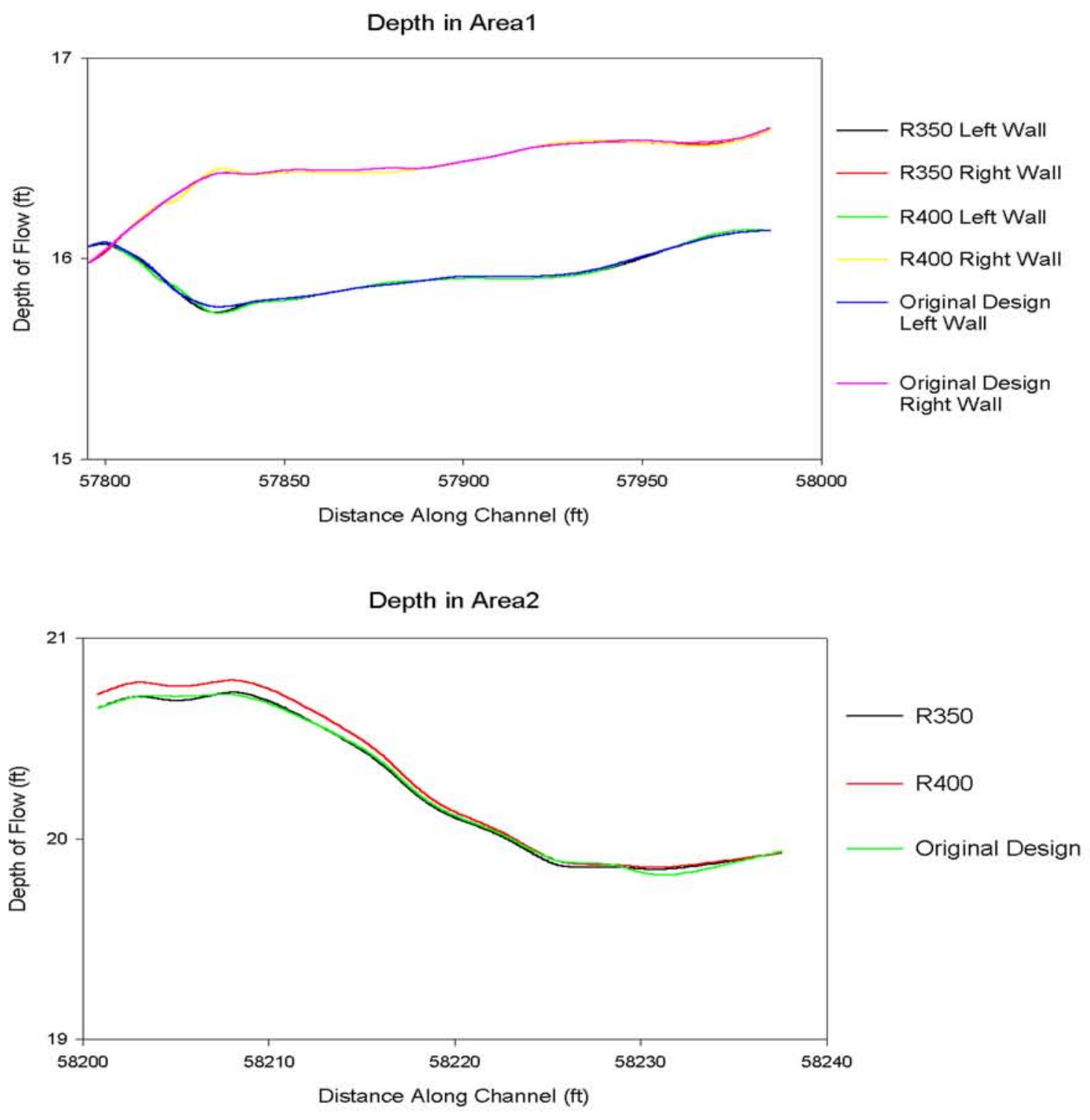

Figure 4.13: Depth Profiles in Area1 and Area2 for Radius of Curvature. 
Table 4.3: Radius of Curvature Parameter Sensitivity Objective Function Values

\begin{tabular}{|c|c|c|c|c|}
\hline \multirow{2}{*}{ Run Name } & \multicolumn{2}{|c|}{ Objective Function 1 } & \multicolumn{2}{c|}{ Objective Function 2 } \\
\cline { 2 - 5 } & Area1 & Area2 & Area1 & Area2 \\
\hline \hline Finaldes & 1.145559 & 1.576477 & 12586.15 & 5357.123 \\
\hline R350 & 1.136146 & 1.604369 & 12585.86 & 5354.309 \\
\hline R400 & 1.151916 & 1.835092 & 12583.6 & 5374.444 \\
\hline
\end{tabular}

A third area examined deals with the width of the San Ramon Channel. This channel has an initial width of 32 feet upstream and contracts to 23 feet upstream from the junction with Walnut Creek. Widths of 20 feet and 25 feet are tested. The widths are adjusting by moving the wall farthest from Walnut Creek only. This is done to avoid introducing any adverse affects from changing the divider wall. The transition from 32 feet to the new width became unsymmetrical as a result, but the affects were negligible. Figure 4.14 shows the mesh outline and bed elevations for the two test cases. The resulting objective function values are given in Table 4.4. Figure 4.15 shows the resulting water surface elevations for channel width of 20 feet, 25 feet, and the original width of 23 feet. The resulting depths are shown in Figure 4.16. The objective functions indicate that the narrower the channel the better the design. However, with a narrower channel come higher depths. The wall heights in San Ramon are 15 to 22 feet. The depths in San Ramon Bypass Channel for the 20-foot wide problem are too near the top of the walls. Without knowing the cost of moving sections of the wall, the decision is made to use the results from the physical model study, that is, a channel width of 23 feet. 


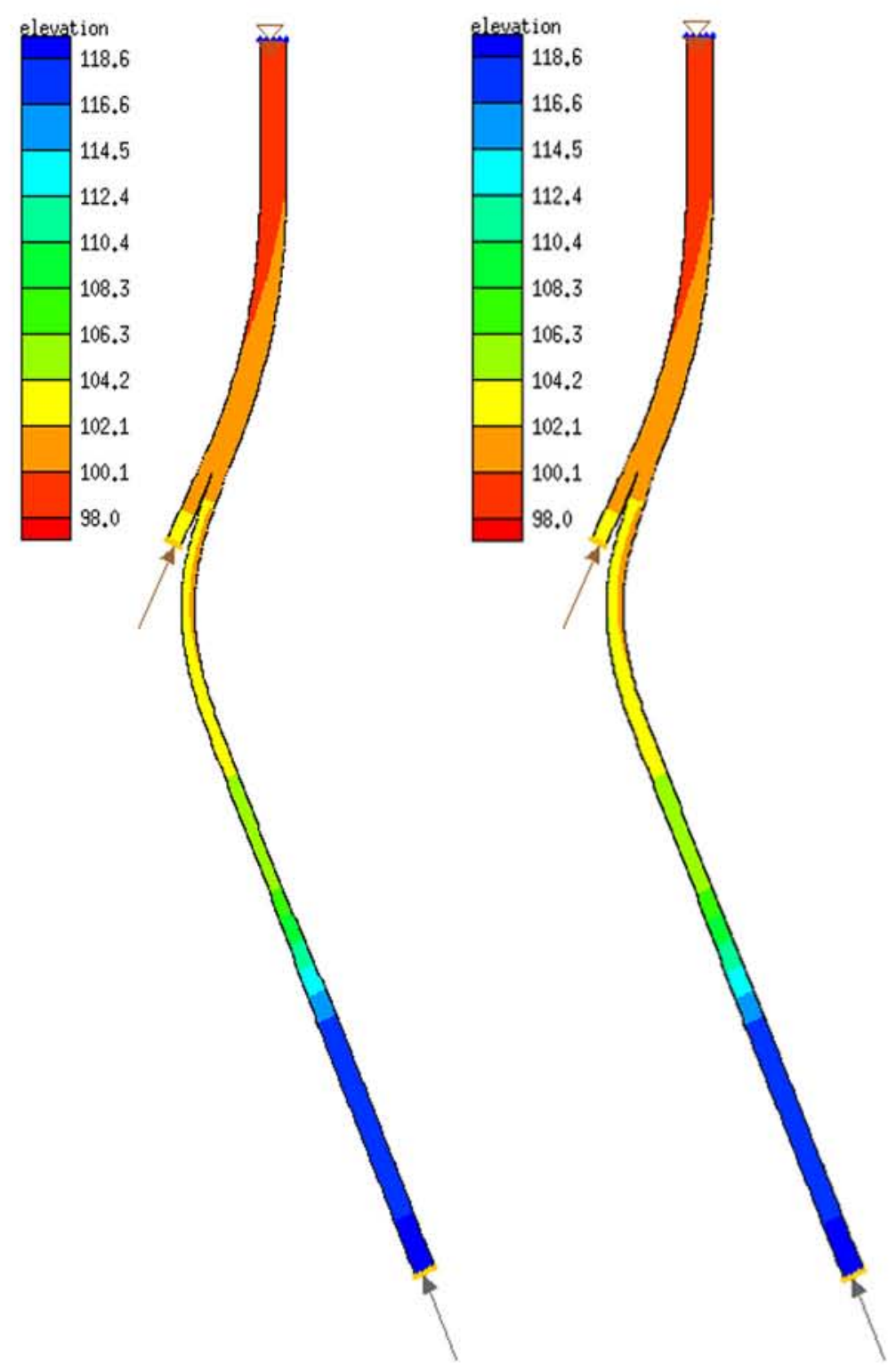

Figure 4.14: Bed Elevations for Channel Widths of 20' (left) and 25' (right). 

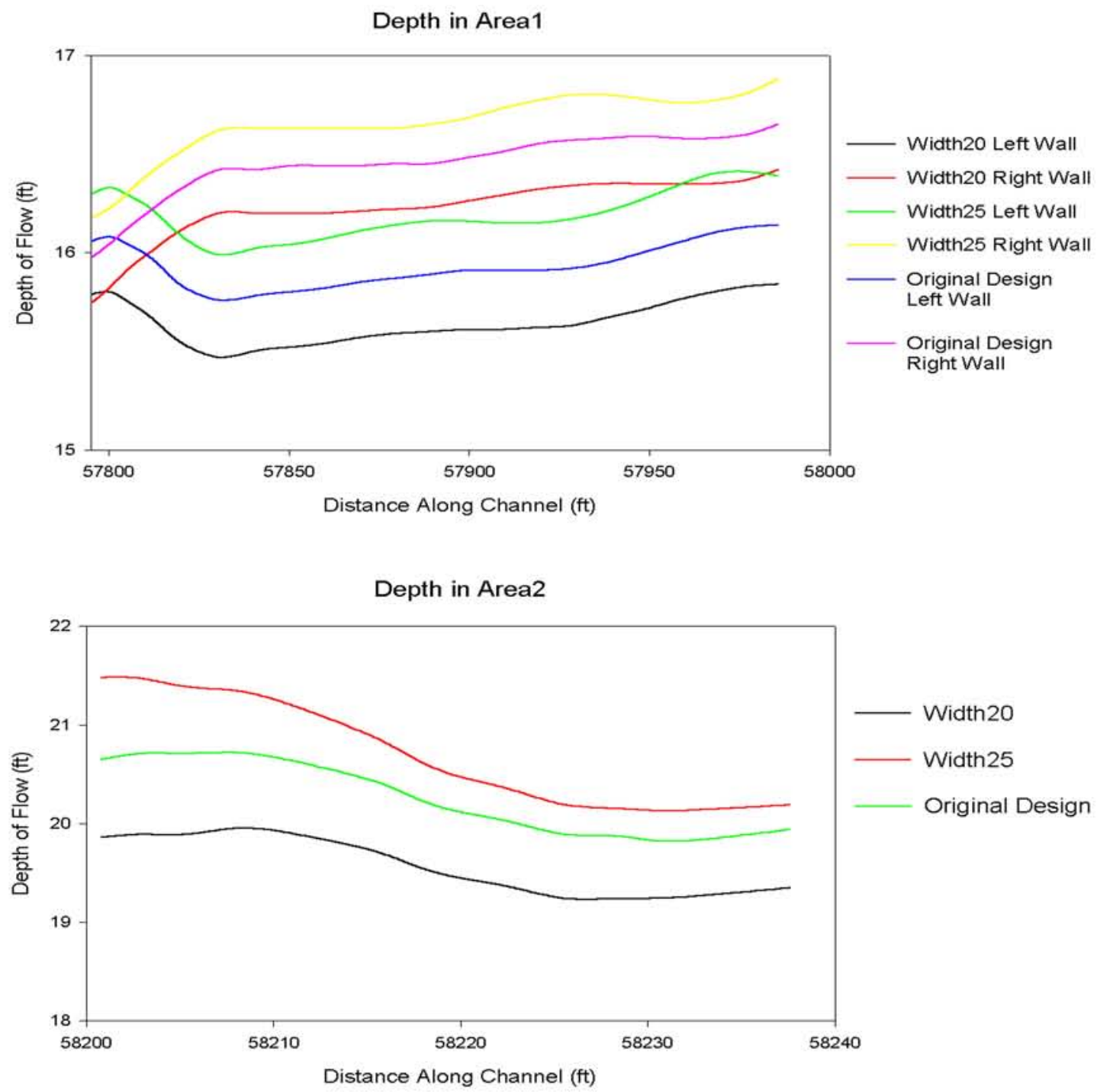

Figure 4.15: Water Depth Profiles in Area1 and Area2 for Width Test Cases. 

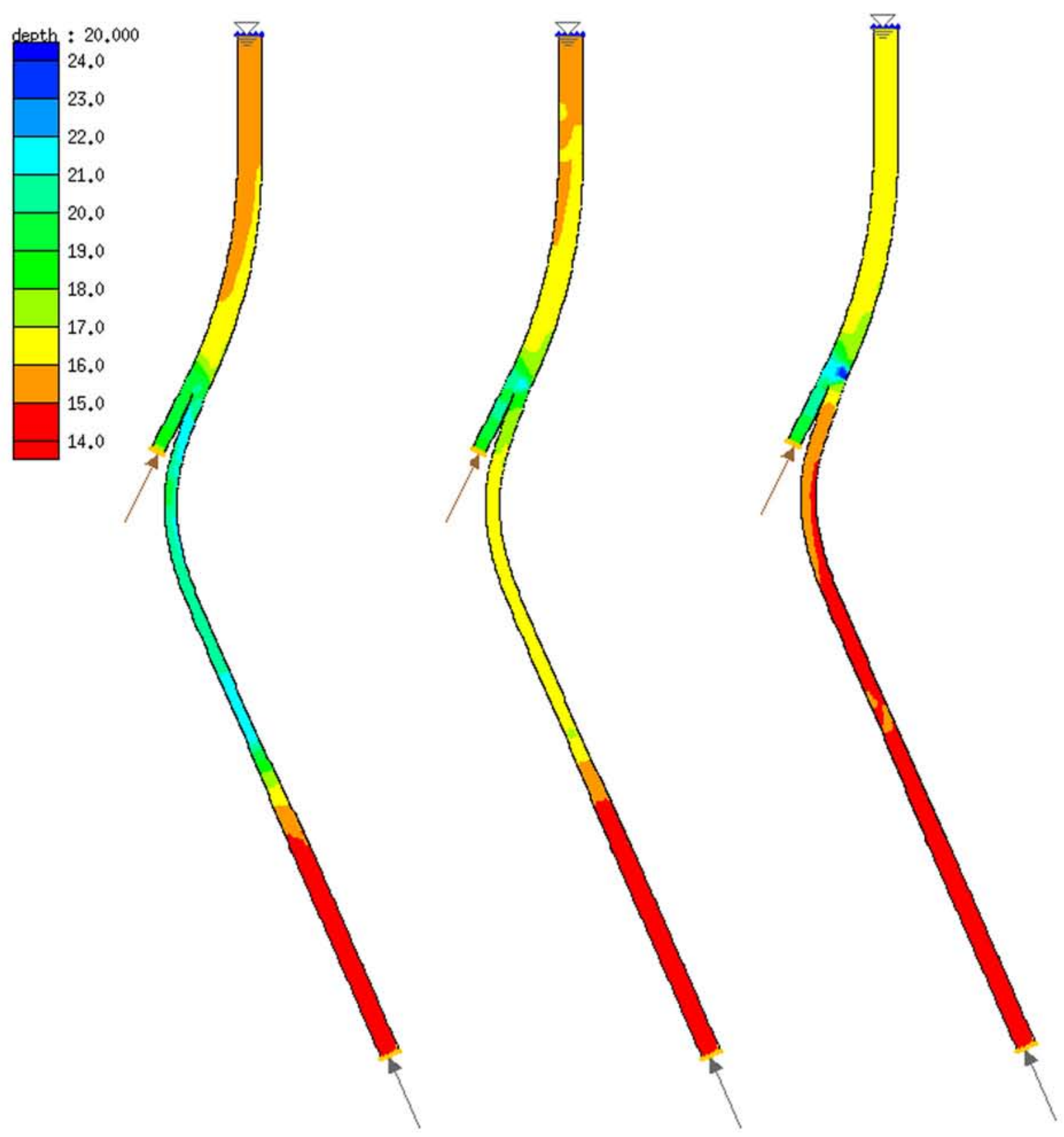

Figure 4.16: Depths for Channel Widths of 20' (left), 23' (center), and 25' (right). 
Table 4.4: Channel Width Parameter Sensitivity Objective Function Values

\begin{tabular}{|c|c|c|c|c|}
\hline \multirow{2}{*}{ Run Name } & \multicolumn{2}{|c|}{ Objective Function 1 } & \multicolumn{2}{c|}{ Objective Function 2 } \\
\cline { 2 - 5 } & Area1 & Area2 & Area1 & Area2 \\
\hline \hline Width20 & 1.335859 & 0.9924769 & 12203.65 & 4991.545 \\
\hline Width23 & 1.145559 & 1.576477 & 12586.15 & 5357.123 \\
\hline Width25 & 1.284566 & 3.663777 & 12946.4 & 5610.11 \\
\hline
\end{tabular}

The final area explored is the length of the divider wall extension located at the confluence. Test with lengths of the divider wall at 10 feet, 30 feet, 40 feet, and 63.8 feet were performed. Table 4.5 shows that the objective functions are sensitive to the changes in the divider wall extension lengths. The values of objective function 1 in area 1 indicate that the longer divider wall lengths are better. However, objective function 2 in area 2 indicates just the opposite. Figure 4.17 shows the resulting water surface profiles for the divider wall test cases. Examination of the resulting depths reveals the reasoning. It is possible to generate a smooth water surface elevation while increasing the depth. Subcritical flow has an increased depth but a smoother water surface. While using an objective function that considers the roughness of the water surface, it is possible to overlook increased depth. The results show a hydraulic jump located in area 2. As the divider wall is lengthened, the jump spreads further upstream. The jump eventually spreads throughout area 2 resulting in a smoother water surface. Overtopping occurs, however, because the depth is increased. The length of the divider wall can be determined using sensitivity analysis. For this reason the divider wall length determined by the 
physical model study is used for this study, and the length of the divider wall is not optimized.

Table 4.5: Divider Wall Length Parameter Sensitivity Objective Function Values

\begin{tabular}{|c|c|c|c|c|}
\hline \multirow{2}{*}{ Run Name } & \multicolumn{2}{|c|}{ Objective Function 1 } & Objective Function 2 \\
\cline { 2 - 5 } & Area1 & Area2 & Area1 & Area2 \\
\hline \hline DW10 & 3.27303652 & 429.7831586 & 19958.786 & 7223.307 \\
\hline DW30 & 3.449422665 & 284.275526 & 19652.89796 & 12108.97285 \\
\hline DW40 & 3.212145535 & 178.1307028 & 19694.6363 & 12442.91466 \\
\hline DW64 & 6.664623238 & 4.86675199 & 19289.05433 & 14005.4 \\
\hline
\end{tabular}

The parameter sensitivity analysis indicate that the parameters that have the most impact on Area1 and Area2 are the width of San Ramon Bypass Channel and the length of the divider wall extension. The super elevation and the radius of curvature of the curve section in San Ramon Bypass Channel have a minimal impact on the model globally. However, the results from the sensitivity analysis for the width of San Ramon Bypass Channel indicate that the channel should be as narrow as possible without going subcritical to achieve a better design. Narrower channels, however, lead to higher depths in San Ramon Bypass Channel and potential overtopping. The depths for the channel width of 20 feet are too close to the top of the walls. To ensure that an appropriate factor of safety is met, the channel width of 23 feet is used. Likewise, the divider wall sensitivity study also points to an answer. The longer the divider wall, the better the flow downstream from the confluence. However, if the wall is too long, a hydraulic jump is pushed upstream which eventually overtops the channel wall. The suggested design is 

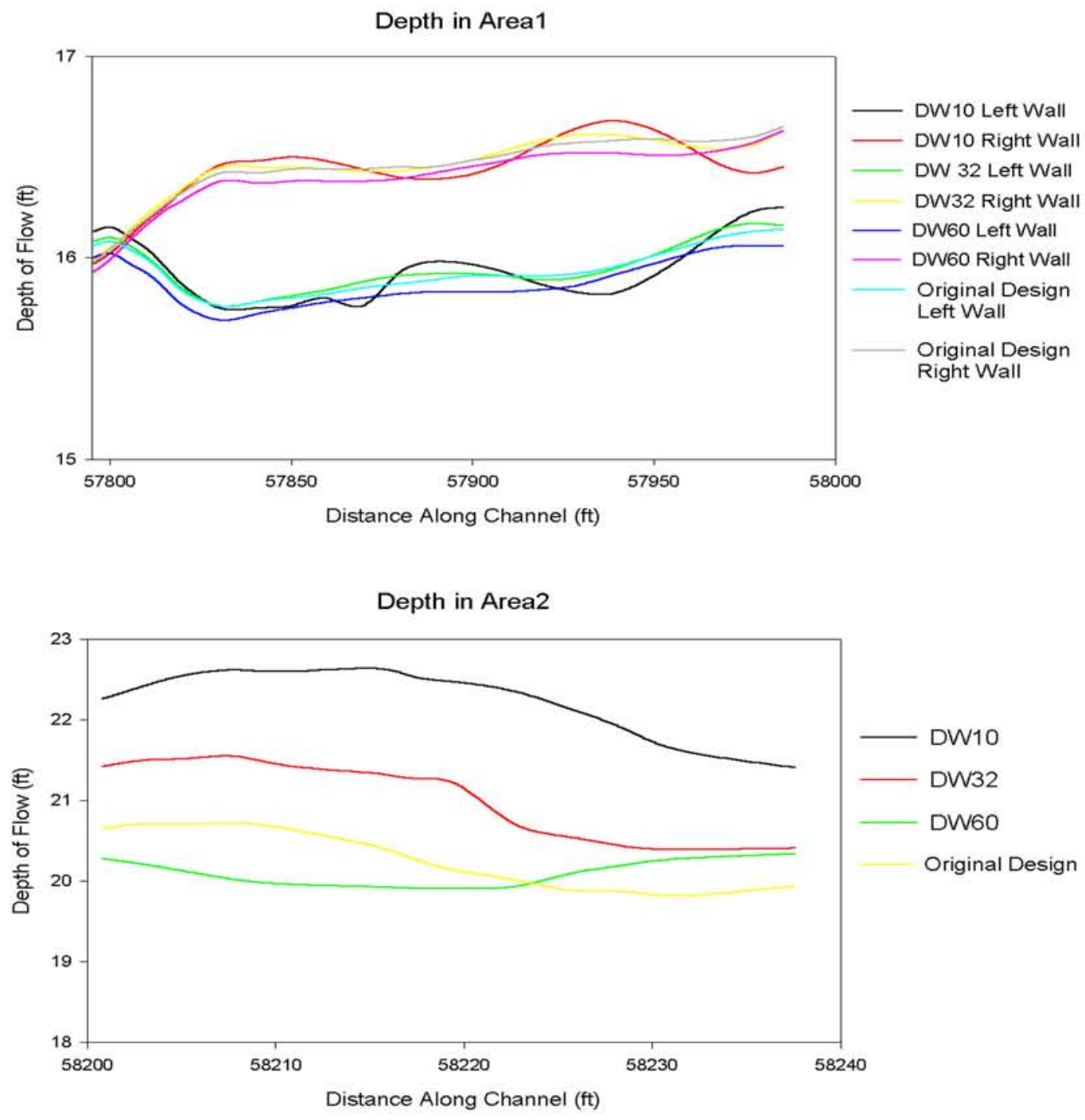

Figure 4.17: Water Depth Profiles in Area1 and Area2 for Divider Wall Test Cases. 
to lengthen the divider wall until the hydraulic jump is within a given distance from the shorter channel wall. Though the results for the sensitivity analysis of the length of the divider wall has an effect globally on the model, without more information about constraints in the area like factor of safety with regards to the wall heights it is not possible to determine a more adequate design than that determined by the physical model study.

The model is locally sensitive to the super elevation and the radius of curvature of the curved section in San Ramon Bypass Channel. That is, changes to the super elevation and the radius of curvature create great disturbances in the curved section in San Ramon Bypass Channel. These parameters can be optimized locally. That is the design considerations for these two parameters are in the San Ramon Bypass Channel only. This work uses optimization techniques to determine a better design for the super elevation and radius of curvature of the curve in the San Ramon Bypass Channel. The results are discussed in the following chapter.

\subsection{Moving the Grid}

A Laplacian smoothing technique, illustrated in the previous chapter, is used to smooth the grid once the boundaries have been moved. The difficulty is in locating the boundaries for the given changes to the design parameter. For the super elevation problem, the z-values are adjusted by calculating a percent change for a given length. Once the boundaries are set, the remaining z-values are adjusted using the Laplacian technique. The entrance and exit spirals complicate adjusting the boundaries for the radius of curvature problem. For the radius of curvature problem, the tangent lengths remain constant to insure that the mesh is only moved 
in the area of the current curve. With a given value for the radius of curvature, the lengths of the entrance and exit spirals are obtained using the following equations.

$$
\begin{aligned}
& T_{1}=X_{1}-R * \sin \Delta_{1}+\frac{Y_{2}+R * \cos \Delta_{2}-\left(Y_{1}+R * \cos \Delta_{1}\right) * \cos I}{\sin I} \\
& T_{2}=X_{2}-R * \sin \Delta_{2}+\frac{Y_{1}+R * \cos \Delta_{1}-\left(Y_{2}+R * \cos \Delta_{2}\right) * \cos I}{\sin I}
\end{aligned}
$$

where $T_{1}$ and $T_{2}$ are the tangent lengths for the entrance and exit spirals, respectively, $\mathrm{R}$ is the radius of curvature, $X_{1}$ and $Y_{1}$ are the coordinates of the point of change from the entrance spiral to the curve, $X_{2}$ and $Y_{2}$ are the coordinates of the point of change from the curve to the exit spiral, $\Delta_{1}$ is the central angle of the entrance spiral, and $\Delta_{2}$ is the central angle of the exit spiral [Rubey 38]. The equations are solved using a Newton iterative solver. A layout of the curve is given in Figure 4.18.

\subsection{Circular Curve Problem}

To test the ability of the model to produce reasonable answers, a test case is conducted. The radius of a curve with no spirals or super elevation is expected to increase to generate smoother flow downstream. A model representing the Walnut Creek area is generated with a circular curve with no entrance or exit spirals and no super elevation in the San Ramon Bypass Channel just upstream of the confluence. The initial design is illustrated in Figure 4.19. For this design problem, the radius of the circular curve is the only design variable. The objective function 


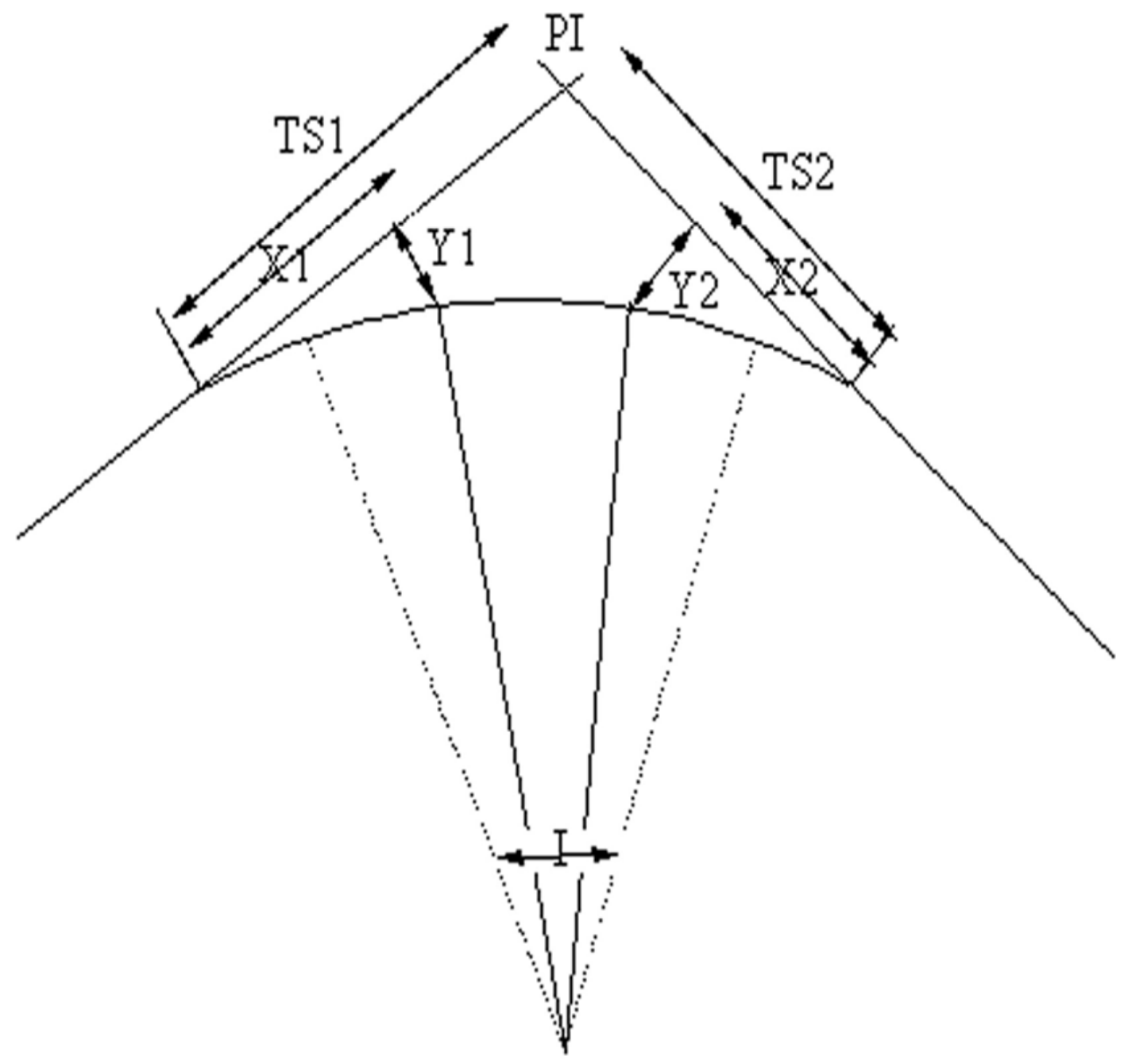

Figure 4.18: Layout of a Circular Curve with Spirals 
is a measure of the roughness or non-uniformity of the water surface in the curve. Figure 4.20 shows the location of the objective function and the design space. The design space consists of a circular curve with two straight sections. The expected optimum would be to push the curve to the design space limits and eliminate the straight sections. This would create a smoother transition, thus reducing the movement of the water.

The radius of the curve is set at 125 feet initially and allowed to move between 0 feet and 400 feet. The points of tangency with the model are allowed to move but are constrained. Figure 4.22 shows the points of tangency for the maximum radius of curvature value. The design process terminates when the design variable (radius) becomes larger than the constraint of 400 feet. There is an upper bound because the end points are fixed. The optimization parameters consist of using the quasi-analytic method to compute the derivatives and the method of steepest descent with a linear search to update the design variable. Figure 4.21 shows the objective function values for the corresponding radius values. The results show that the larger the radius the better the design. This indicates the need for entrance and exit spirals and super elevation.

\subsection{Super Elevation}

When changing the direction of flow via curved channels, the centripetal force of the fluid causes a rise in the water surface on the outside wall and a depression in the water surface along the inside wall. These curves also cause disturbances

in the flow that can persist downstream. To balance the force of the fluid, the channel bed can be sloped across the channel. This transverse bed slope is known as super elevation. 


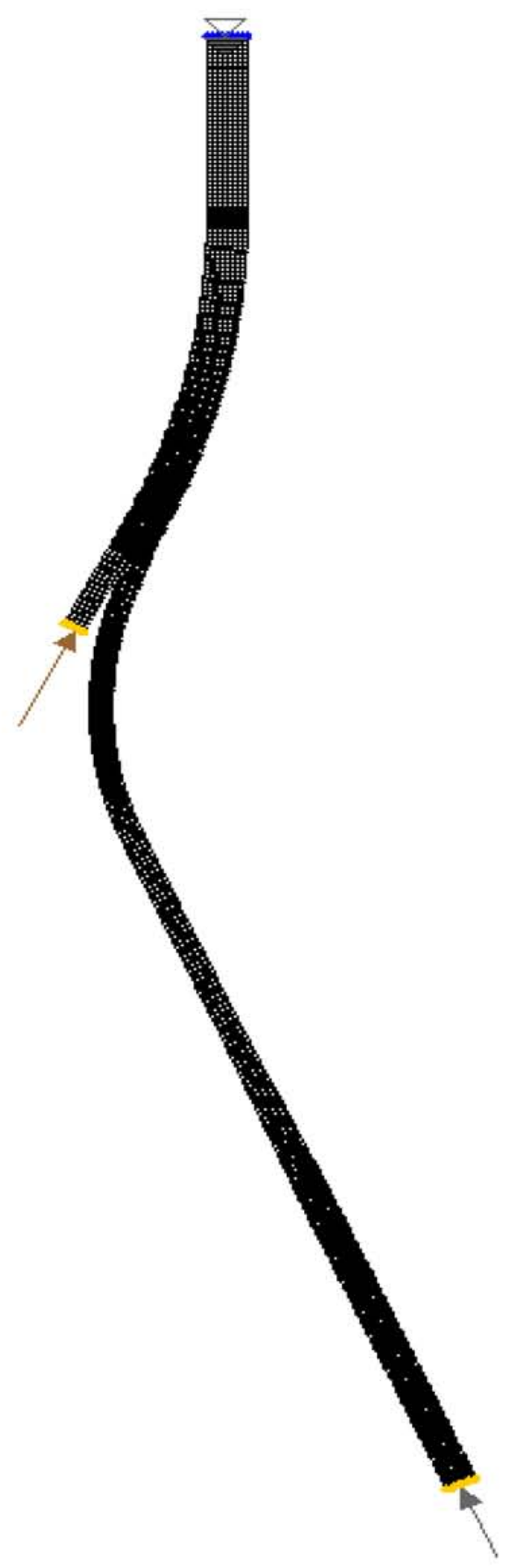

Figure 4.19: Initial Mesh for the Circular Curve Optimization Problem. 


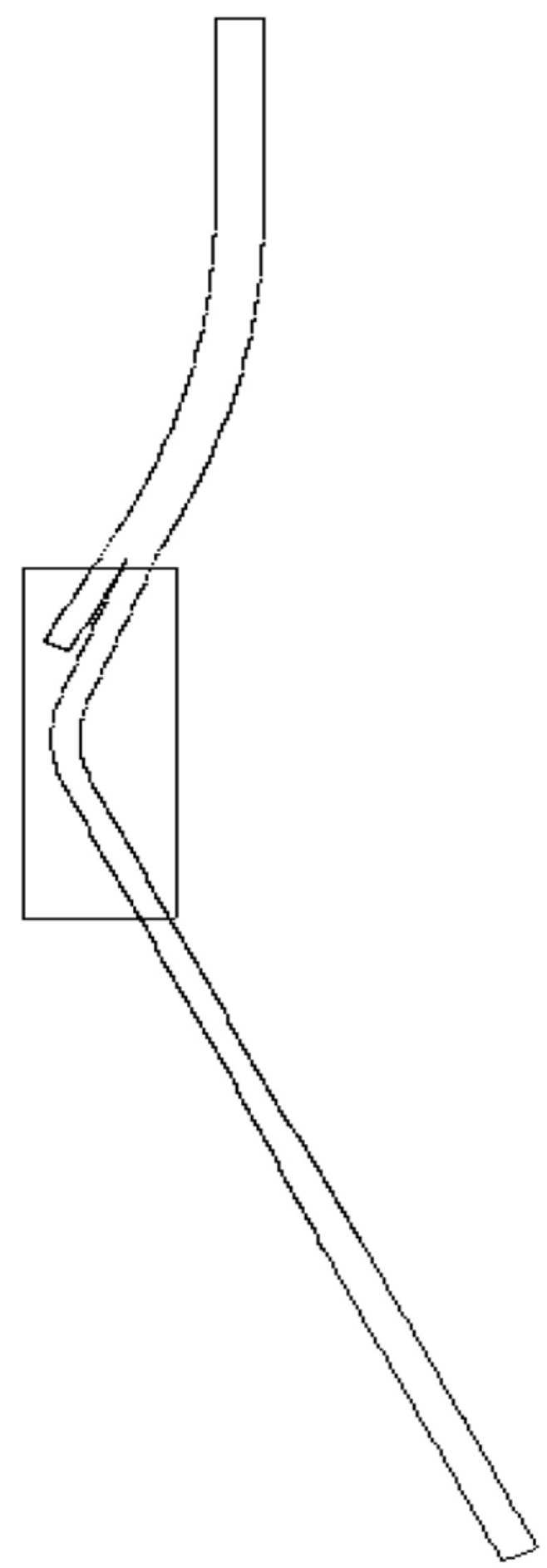

Figure 4.20: Objective Function and Design Space for the Circular Curve Problem. 


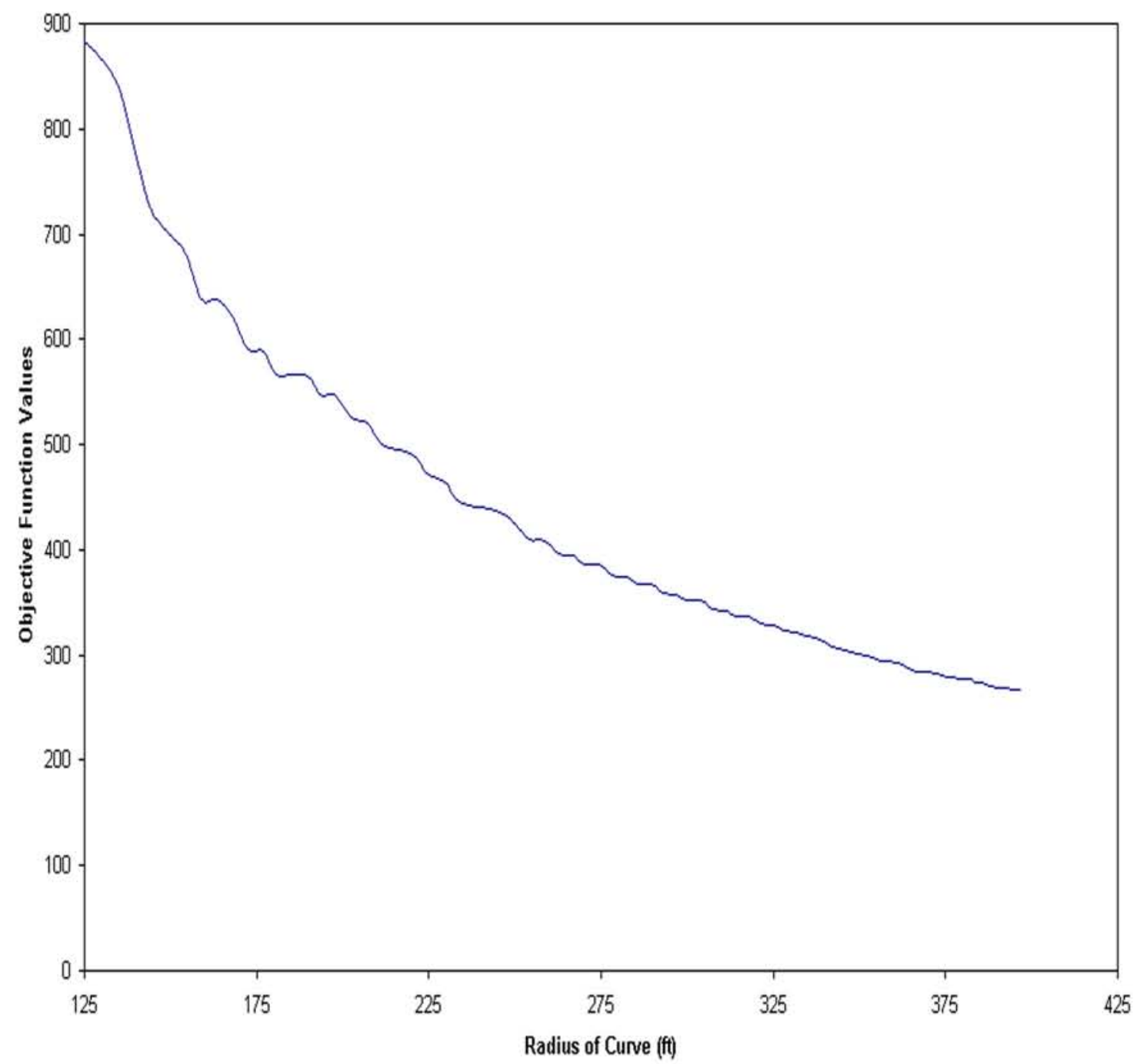

Figure 4.21: Objective Function Values versus Radius of Curvature. 


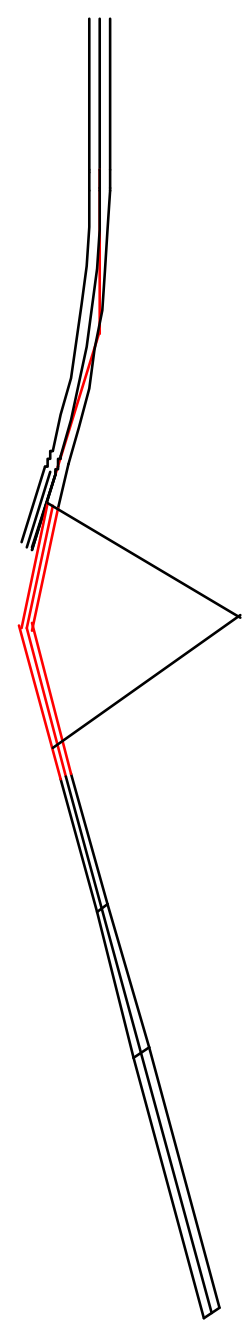

Figure 4.22: Layout of Curve Including Bounds for Points of Tangency. 
The curved section of the San Ramon Bypass Channel, shown in Figure 4.23, changes the flow direction by 43.6 degrees. The flow is supercritical and at a rate of 15,200 cfs. The channel is 23 feet wide in the curved section. The suggested range of super elevation values for these flow and geometry parameters given by Equation 4.3 is between 2.137906202 feet and 4.2758124 feet [EM 1110-2-1601]. This is based on the assumption that the velocity distribution is uniform across the channel and that the radius of curvature is 285 feet for the entire curve. However, the velocity distribution is not likely to be uniform across the channel and the radius of curvature varies from 273.5 feet and 296.5 feet across the channel. The super elevation determined by the physical model study was 3.82 feet.

$$
\Delta y=C \frac{V^{2} W}{g r}
$$

where

$\Delta y=$ super elevation

$\mathrm{C}=$ coefficient between 0.5 and 1.0

$\mathrm{V}=$ mean channel velocity

$\mathrm{W}=$ channel width

$\mathrm{g}=$ acceleration of gravity

$\mathrm{r}=$ radius of curvature to center line

The design variable is the super elevation in the curve in San Ramon Bypass Channel (Figure 4.24). The problem is optimized using Equation 3.1 as the objective function. The objective space is located along the walls in the downstream section of the curve and just downstream of the curve as illustrated in 


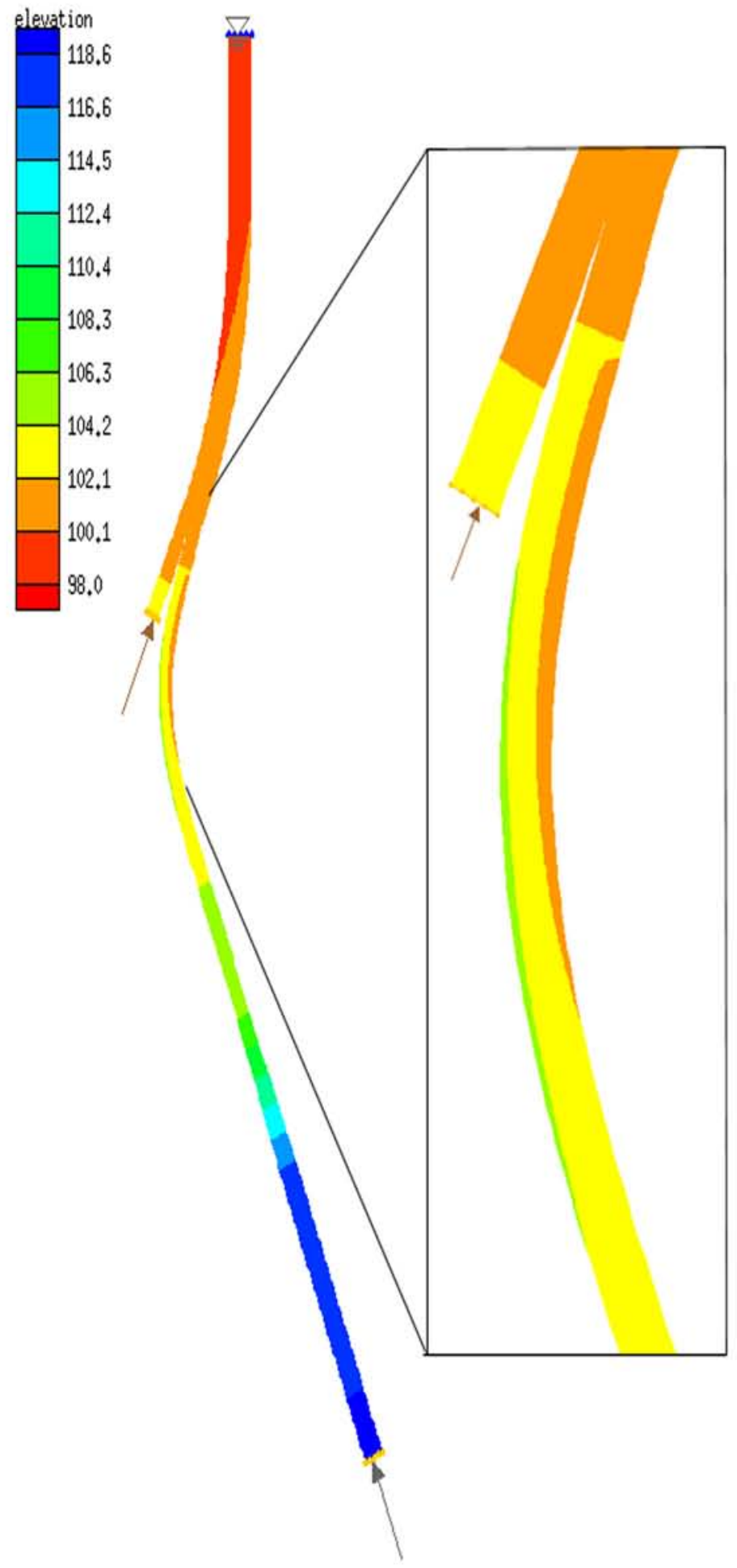

Figure 4.23: Initial Grid for the Super Elevation Problem. 
Figure 4.25. The initial design has a super elevation value of 2.14 feet. The design iterations are plotted against the design variable in Figure 4.26. The improved solution is determined within nine design iterations. The resulting value for super elevation is 3.35 feet and is illustrated in Figure 4.27. Comparing this value to the value determined by the physical model, which is 3.82 feet, and the range determined by Equation 4.3, which is 2.138 feet to 4.28 feet, shows that the result from the optimization code is reasonable. Furthermore, comparing the depth profiles for the initial design and new design (Figure 4.28) shows that the new design is an improved design. The maximum water depth is reduced by 0.3 feet. In some locations the depth is reduced by more than 0.5 feet. The objective function value is reduced in the improved design by 30\%. Figure 4.29 shows the depth profile of the physical model design and the improved design. The profiles are very similar and indicate the ability of the optimization code to produce improved channel design. A plot of the design variable versus the objective function is given in Figure 4.30.

\subsection{Radius of Curvature}

Curved channels are used to change the direction of flow. To transition the flow more smoothly, entrance and exit spirals are used to gradually change the direction of flow. A poorly designed curve can cause disturbances in the flow that can persist downstream. The proper combination of entrance and exit spiral lengths and radius of curvatures for the circular curve are required to insure the least amount of disturbances possible.

The curve section of the San Ramon Bypass Channel, shown in Figure 4.39, changes the flow direction by 43.6 degrees. The flow is supercritical and at a 


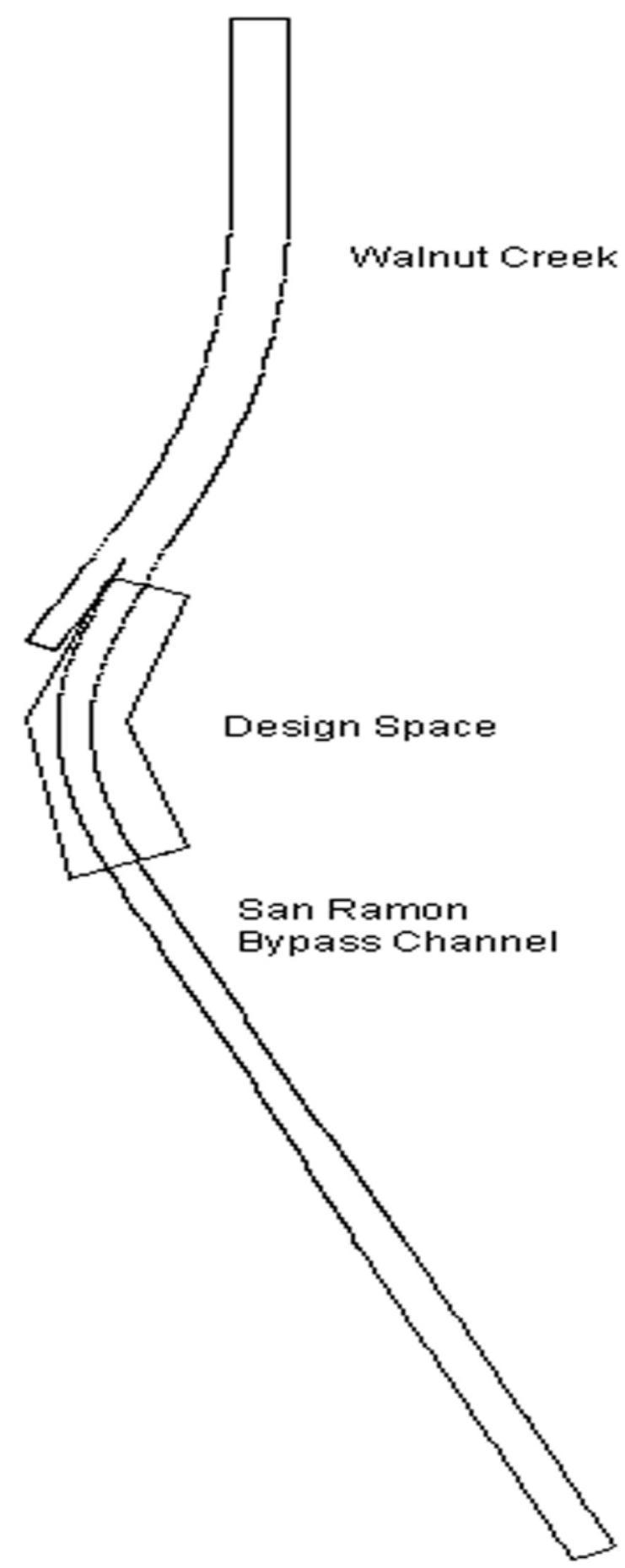

Figure 4.24: The Design Space for the Super Elevation Problem. 


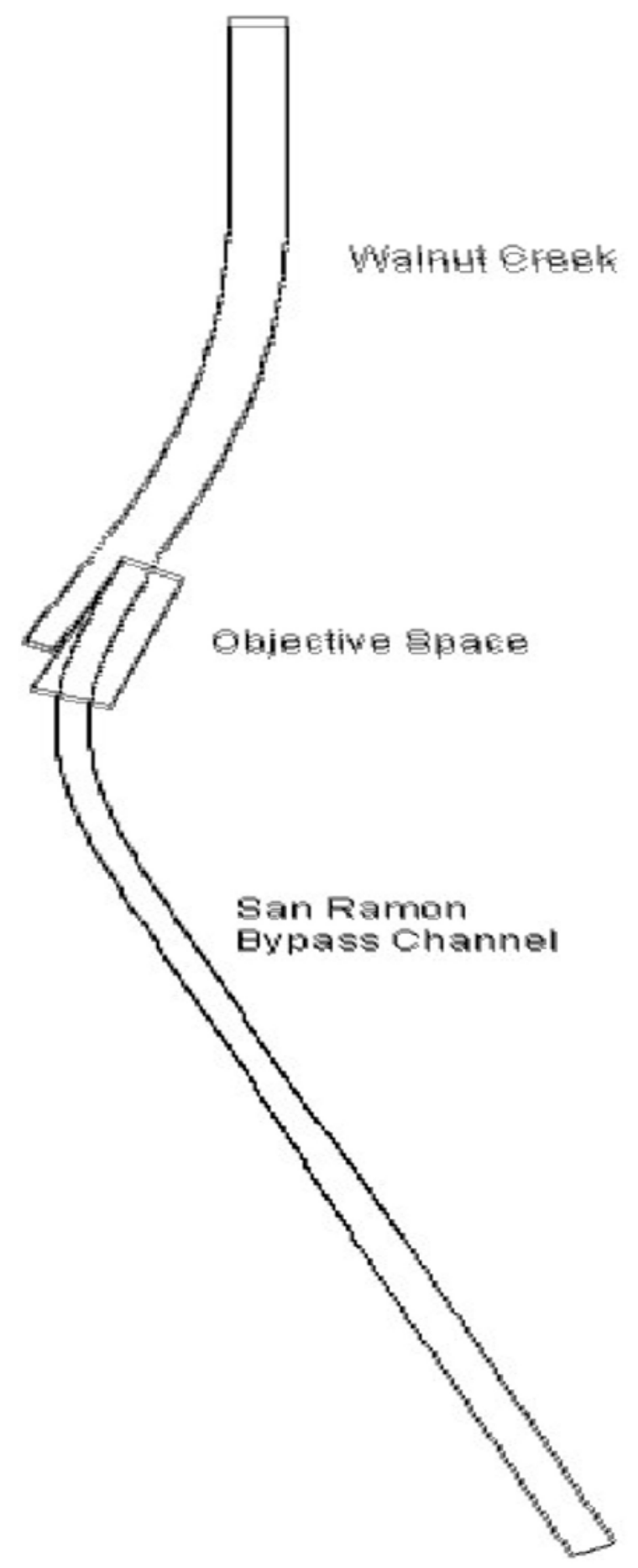

Figure 4.25: The Objective Area for the Super Elevation Problem. 


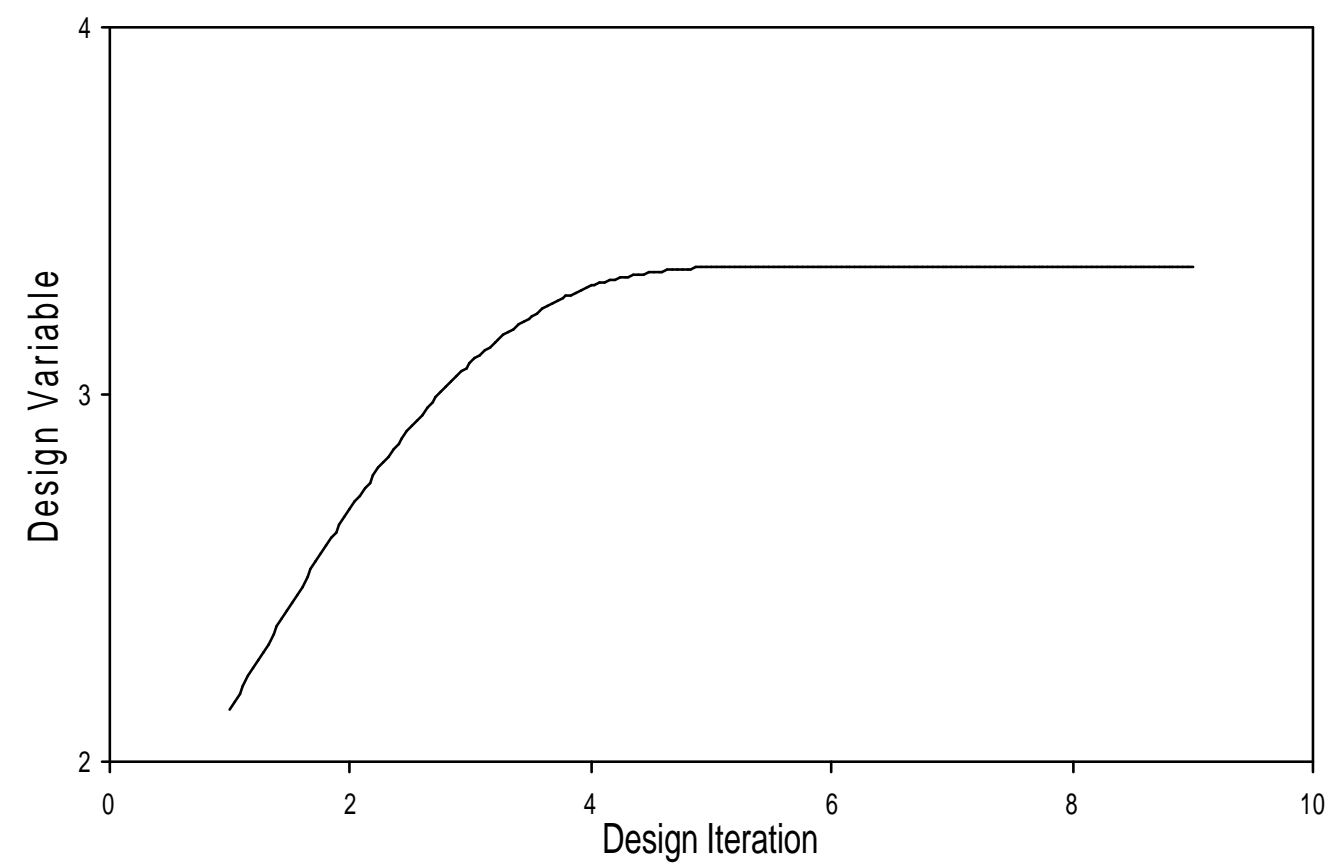

Figure 4.26: Design Parameter versus Design Iteration. 


\begin{tabular}{|c|c|}
\hline elev & 118,6 \\
\hline & 116.6 \\
\hline & 114.5 \\
\hline & 112.4 \\
\hline & $110+4$ \\
\hline & $\begin{array}{l}108+3 \\
106,3\end{array}$ \\
\hline & \\
\hline & 102.1 \\
\hline & 100.1 \\
\hline & 98.0 \\
\hline
\end{tabular}

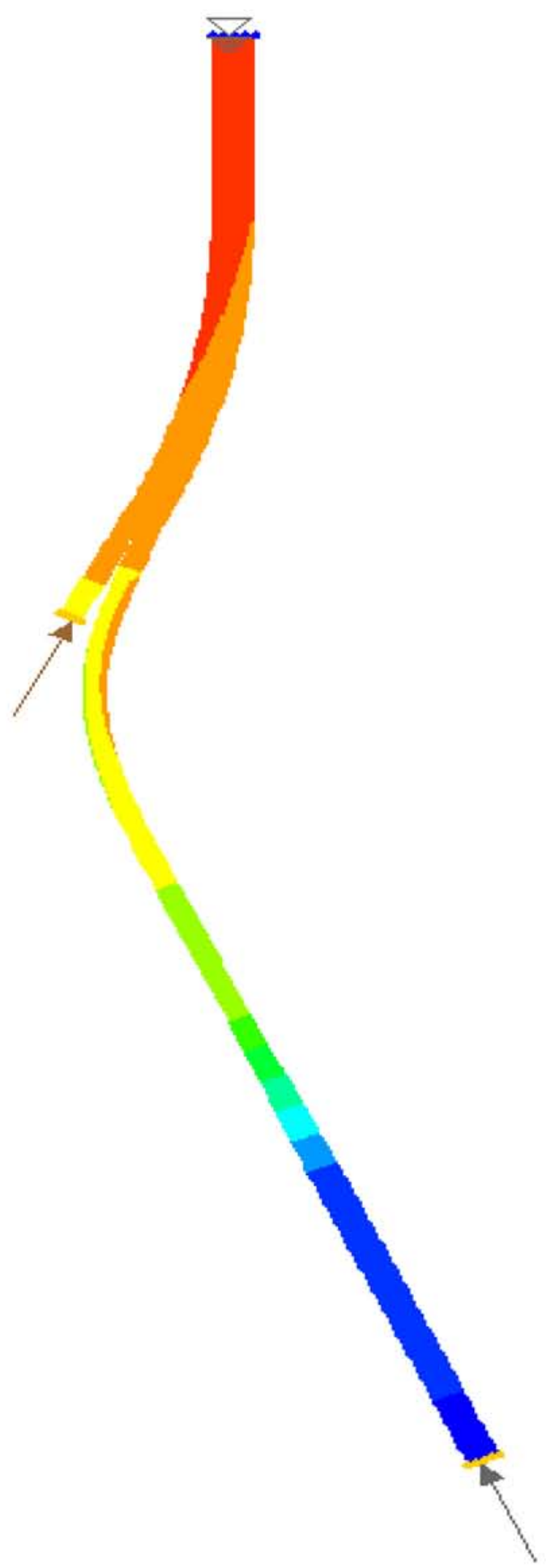

Figure 4.27: Layout and Bed Elevations of the Improved Design. 


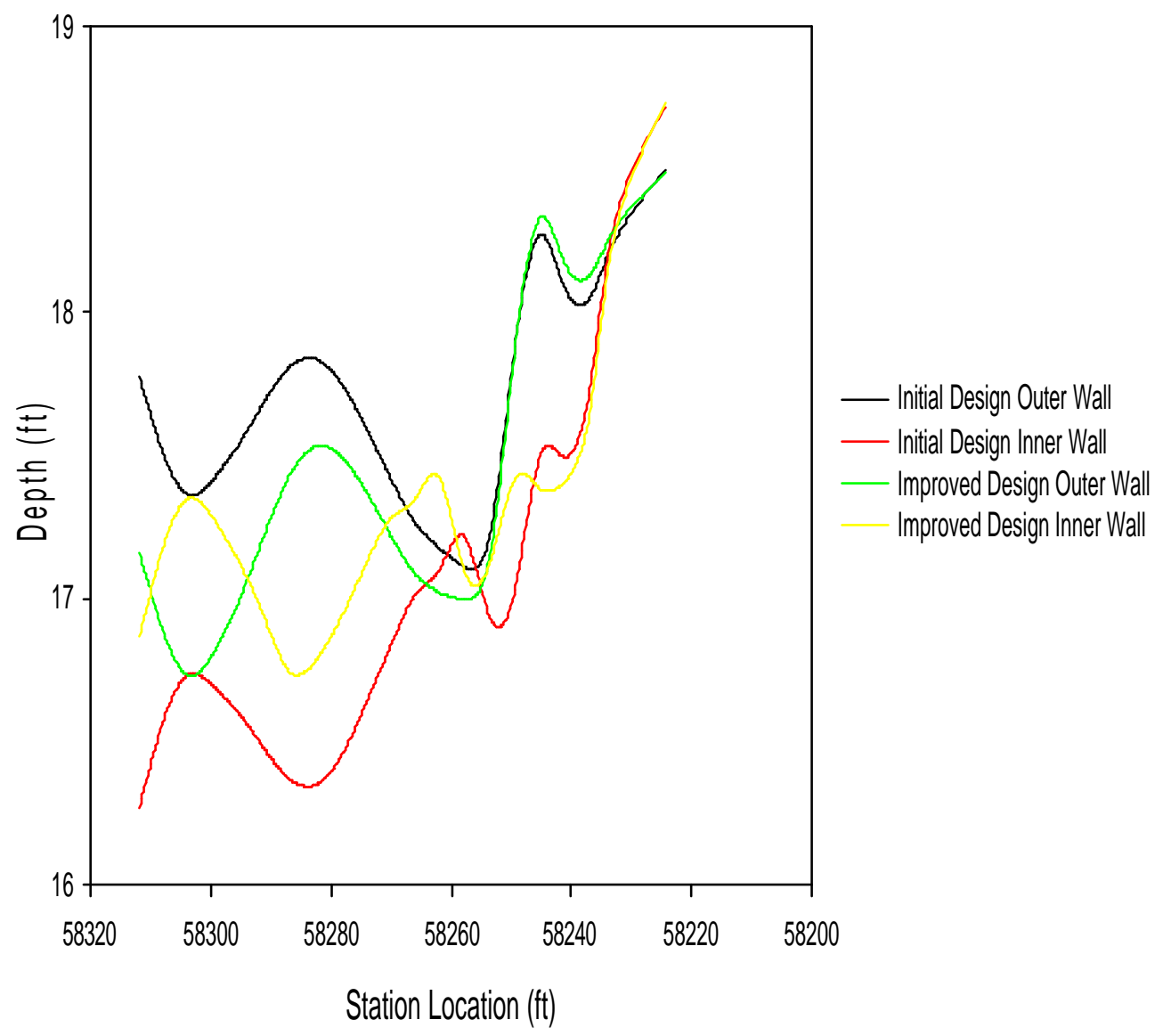

Figure 4.28: Depth Profiles for Initial Design and Improved Design. 


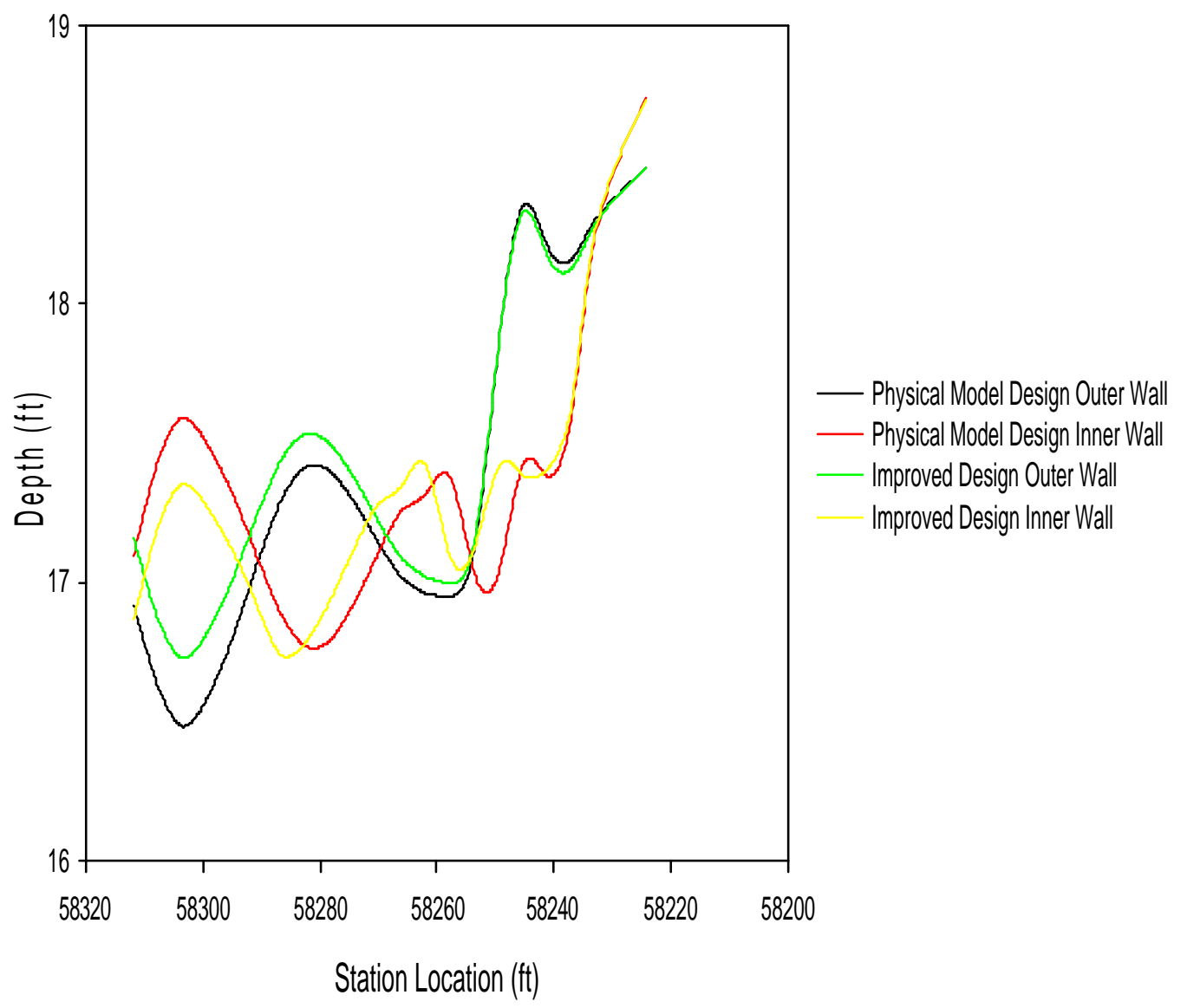

Figure 4.29: Depth Profiles for Physical Model Design and Improved Design. 


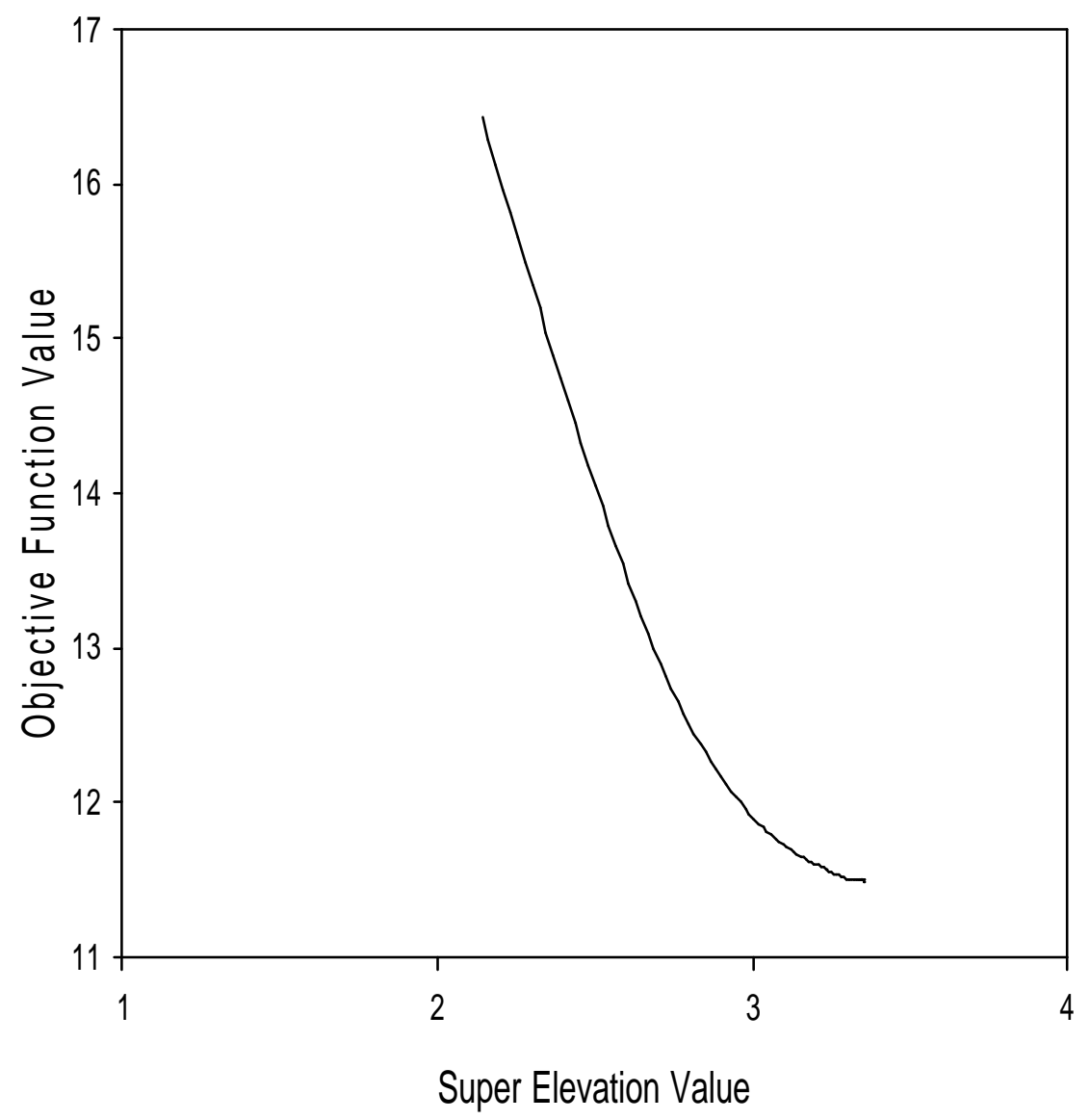

Figure 4.30: Design Variable versus Objective Function. 
rate of $15,200 \mathrm{cfs}$. The channel is 23 feet wide in the curved section. The design determined by the physical model study gives a radius of curvature of 285 feet, entrance spiral length of 200 feet with a tangent length of 209.02 feet, and an exit spiral length of 87.5 feet with a tangent length of 164.99 feet [Davis 87].

The design variable is the radius of curvature in the curve in San Ramon Bypass Channel (Figure 4.32). The problem is optimized using Equation 3.1 as the objective function. The objective space is located along the walls in the downstream section of the curve and just downstream of the curve as illustrated in Figure 4.33. The initial design has a radius of curvature of 325 feet. The design iterations are plotted against the design variable in Figure 4.34. The improved solution is determined within 30 design iterations. The resulting value for radius of curvature is 302 feet and is illustrated in Figure 4.35. Comparing this value to the value determined by the physical model, which is 285 feet, shows that the result from the optimization code is reasonable. Furthermore, comparing the depth profiles for the initial design and new design (Figure 4.36) shows that the new design is an improved design. The maximum water depth is reduced by 0.4 feet. In some locations the depth is reduced by more than 0.5 feet. The objective function value is reduced by $17 \%$. Figure 4.37 shows the depth profile of the physical model design and the improved design. The profiles are very similar and indicate the ability of the optimization code to produce improved channel design. A plot of the design variable versus the objective function is given in Figure 4.38.

\subsection{Radius of Curvature and Super Elevation}

There are several design parameters for a high-velocity channel. The ideal design improvement code would consider all of these parameters when determining 


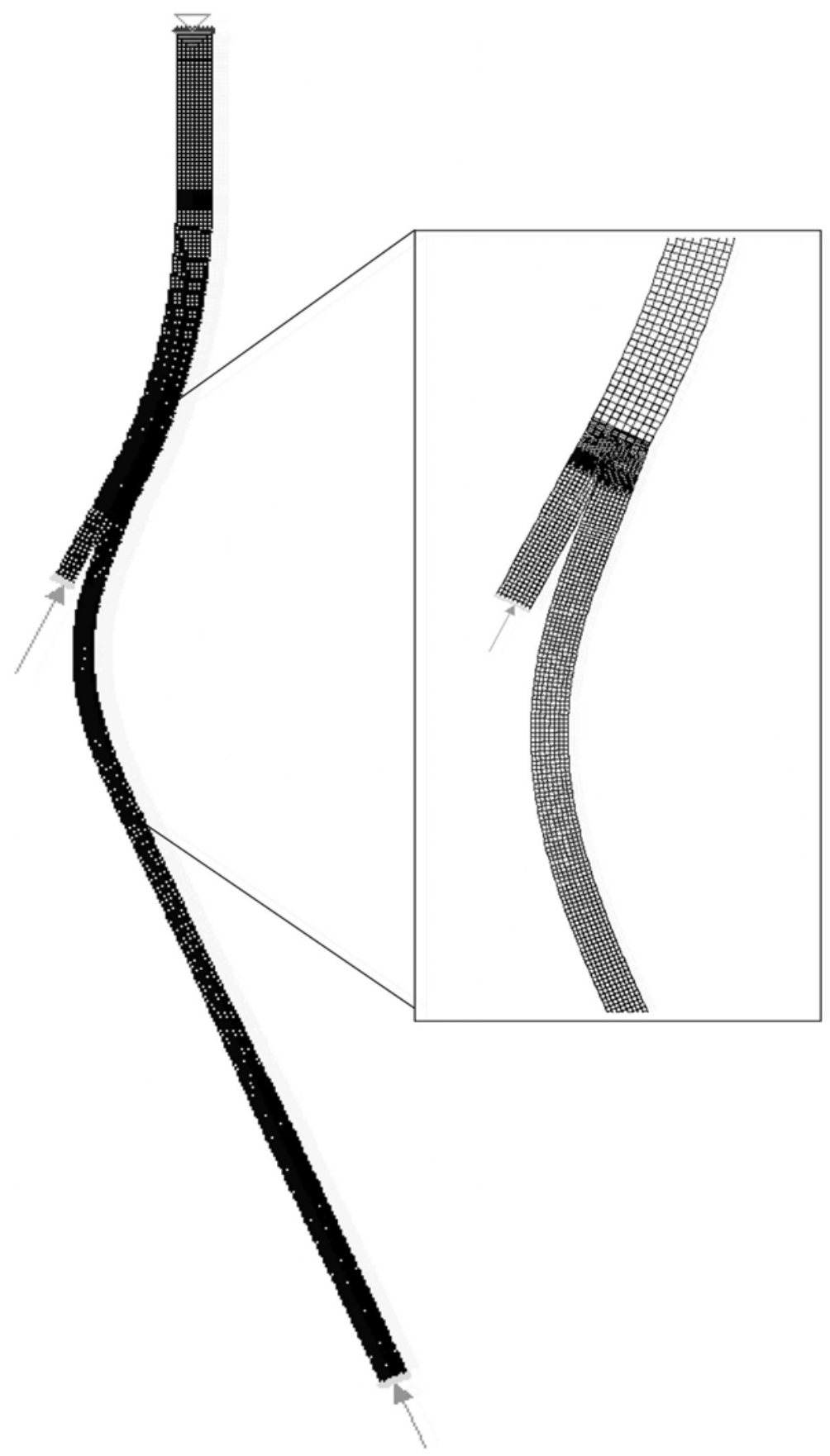

Figure 4.31: Initial Grid for the Radius of Curvature Problem. 


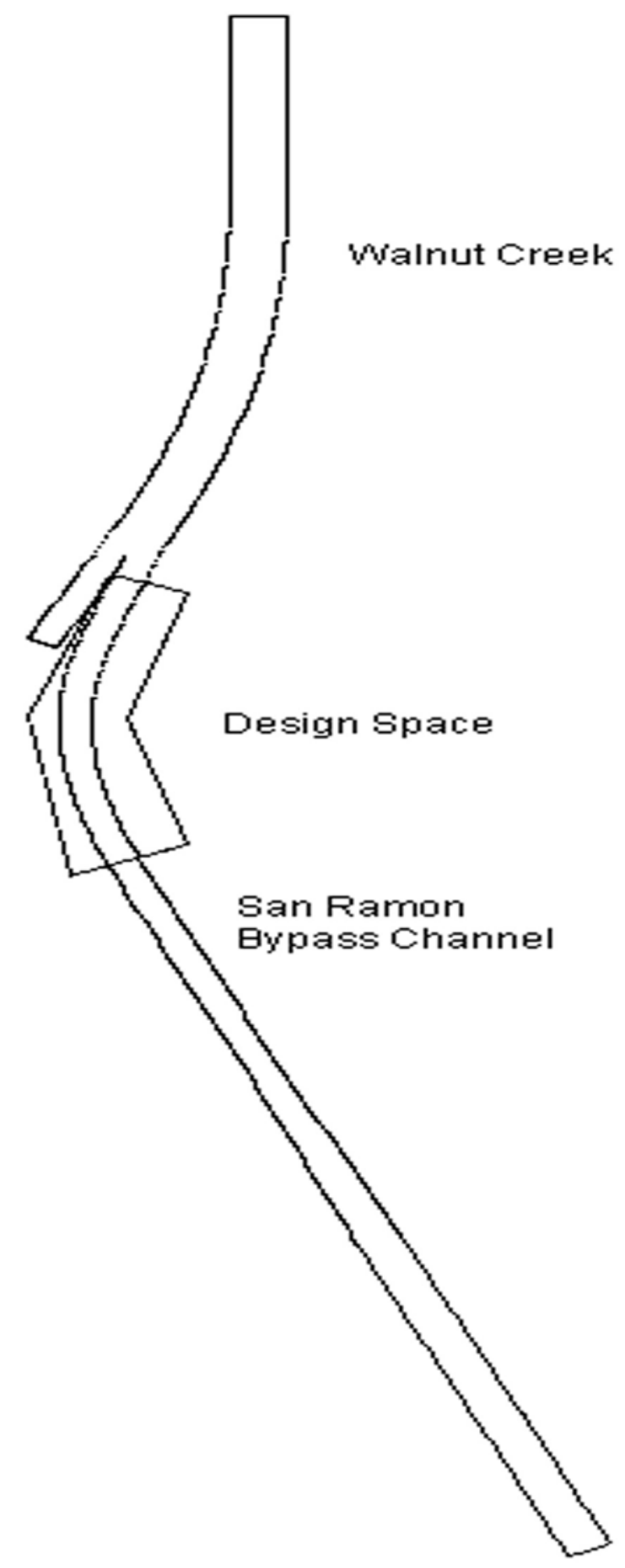

Figure 4.32: The Design Space for the Radius of Curvature Problem. 


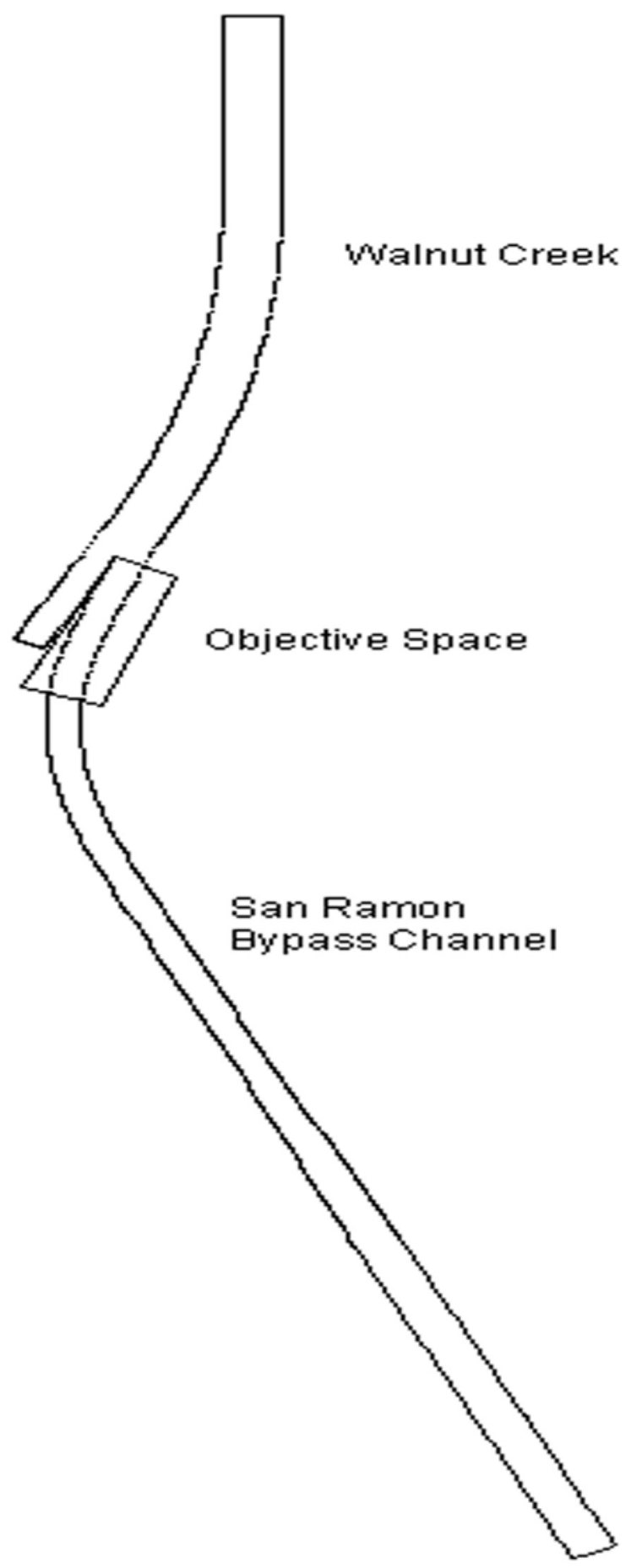

Figure 4.33: The Objective Area for the Radius of Curvature Problem. 


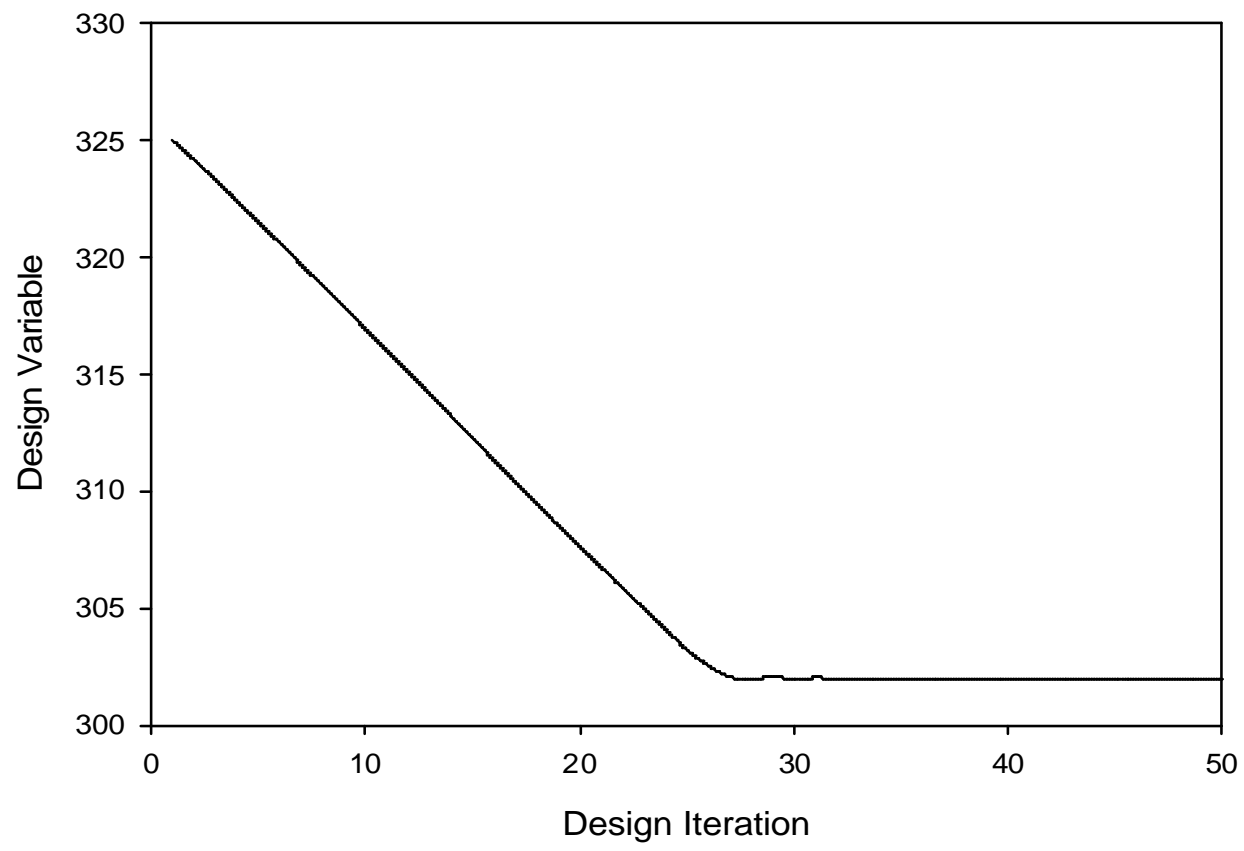

Figure 4.34: Design Variable versus Design Iteration. 


\begin{tabular}{|l|l}
\hline elevation \\
118.6 \\
116.6 \\
114.5 \\
112.4 \\
110.4 \\
108.3 \\
106.3 \\
104.2 \\
102.1 \\
100.1 \\
98.0
\end{tabular}

Figure 4.35: Layout of the Improved Design. 


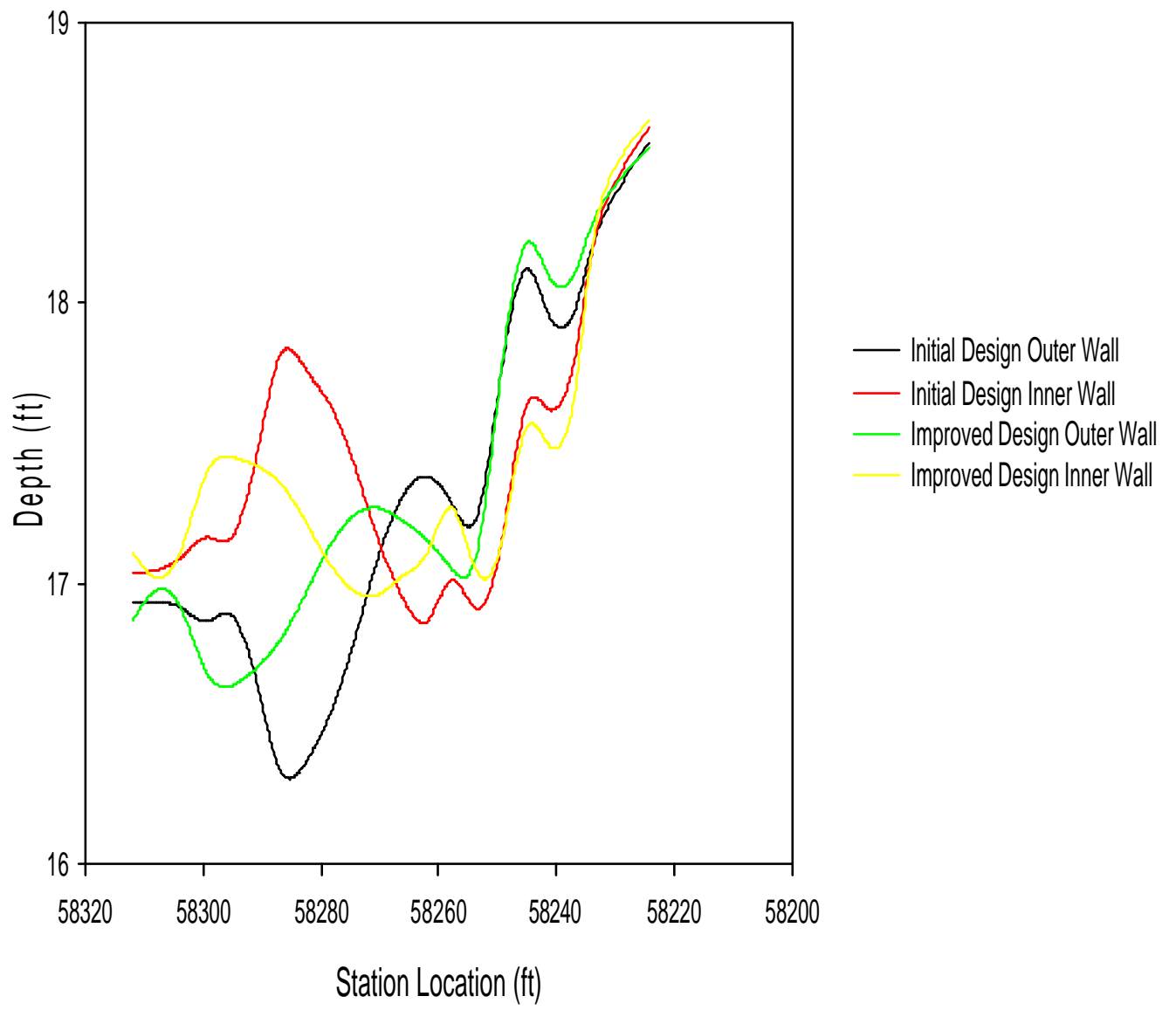

Figure 4.36: Depth Profiles for Initial Design and Improved Design. 


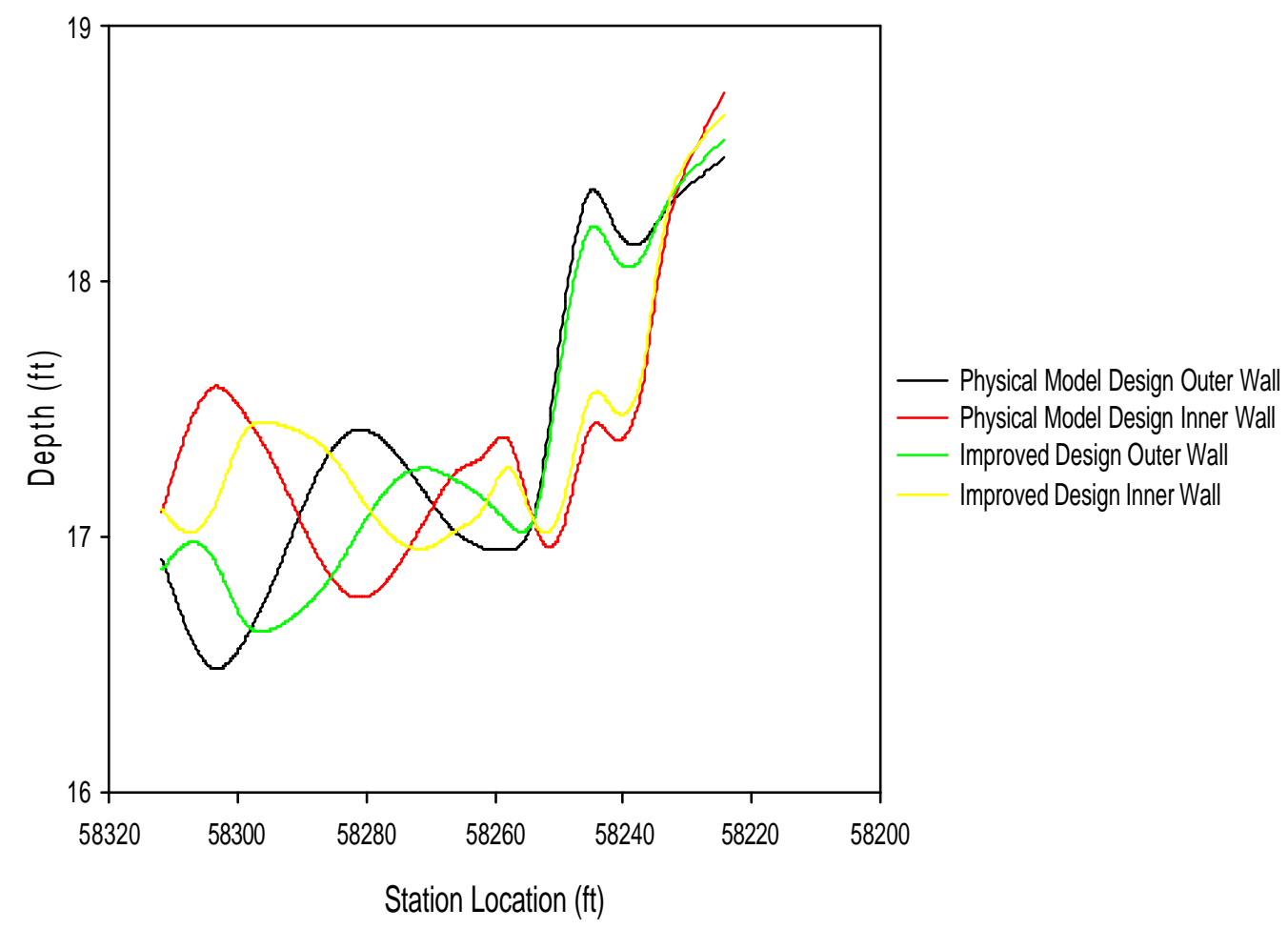

Figure 4.37: Depth Profiles for Physical Model Design and Improved Design. 


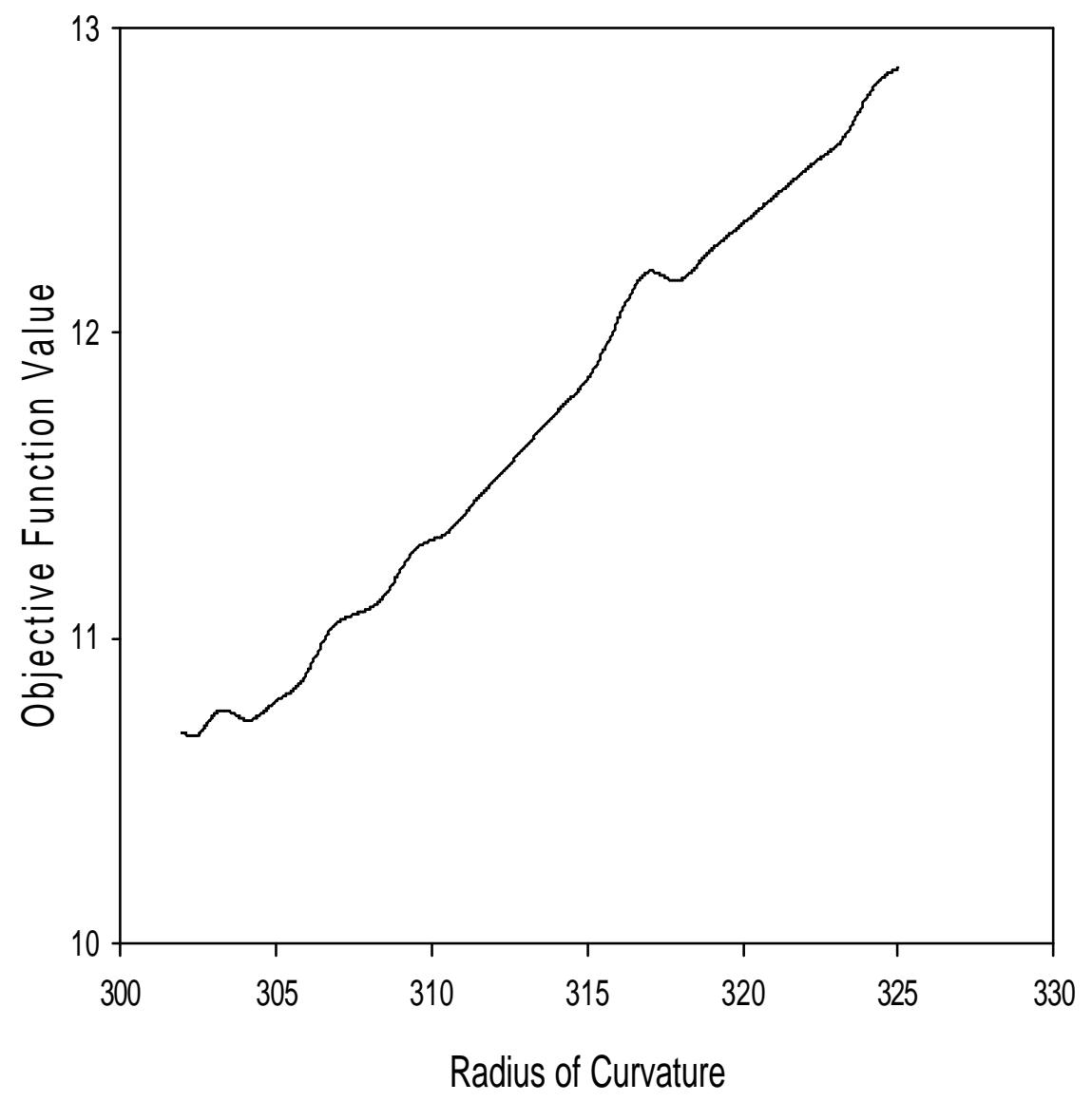

Figure 4.38: Design Variable versus Objective Function. 
a design. To determine the ability of the code to handle multiple design variables, a test was conducted to assess the codes ability to optimize on the radius of curvature and super elevation simultaneously. Figure 4.40 shows the design space for the two design variable problem. The problem is optimized using Equation 3.1 as the objective function. The objective space is located along the walls in the downstream section of the curve and just downstream of the curve as illustrated in Figure 4.41. The design iterations are plotted against the design variable in Figure 4.42. The improved solution is determined within 20 design iterations. The resulting value for radius of curvature is 301.9 feet and is illustrated in Figure 4.43. Comparing this value to the value determined by the physical model, which is 285 feet, shows that the result from the optimization code is reasonable. Furthermore, comparing the depth profiles for the initial design and new design (Figure 4.44) shows that the new design is an improved design. The maximum water depth is reduced by 0.5 feet. In some locations the depth is reduced by more than 0.85 feet. The objective function value is reduced by $40 \%$. Figure 4.45 shows the depth profile of the physical model design and the improved design. The profiles are very similar and indicate the ability of the optimization code to produce improved channel design. A plot of the design variable versus the objective function is given in Figure 4.46, Figure 4.47, and Figure 4.48. 


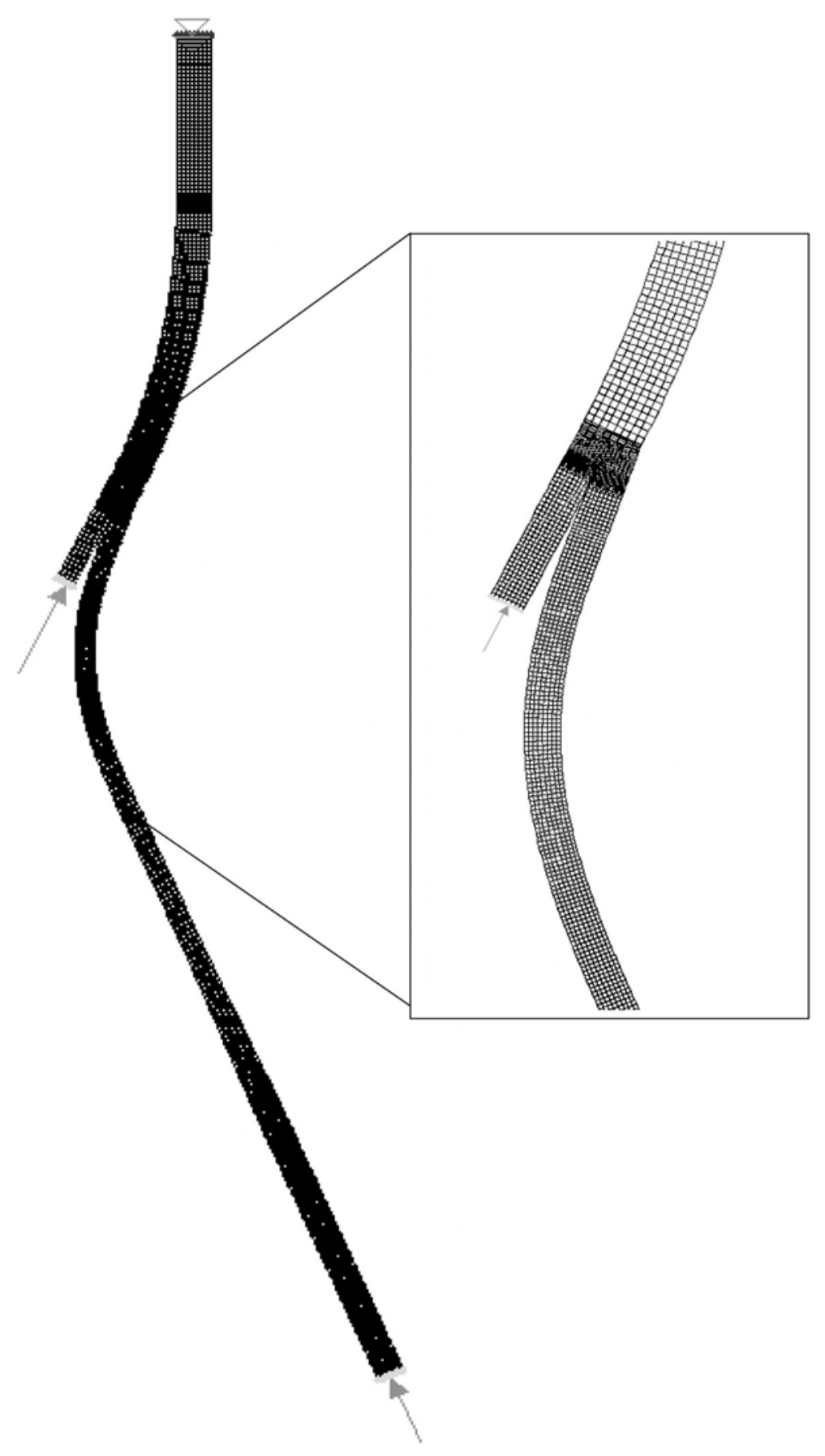

Figure 4.39: Initial Grid for the Radius of Curvature and Super Elevation Problem. 


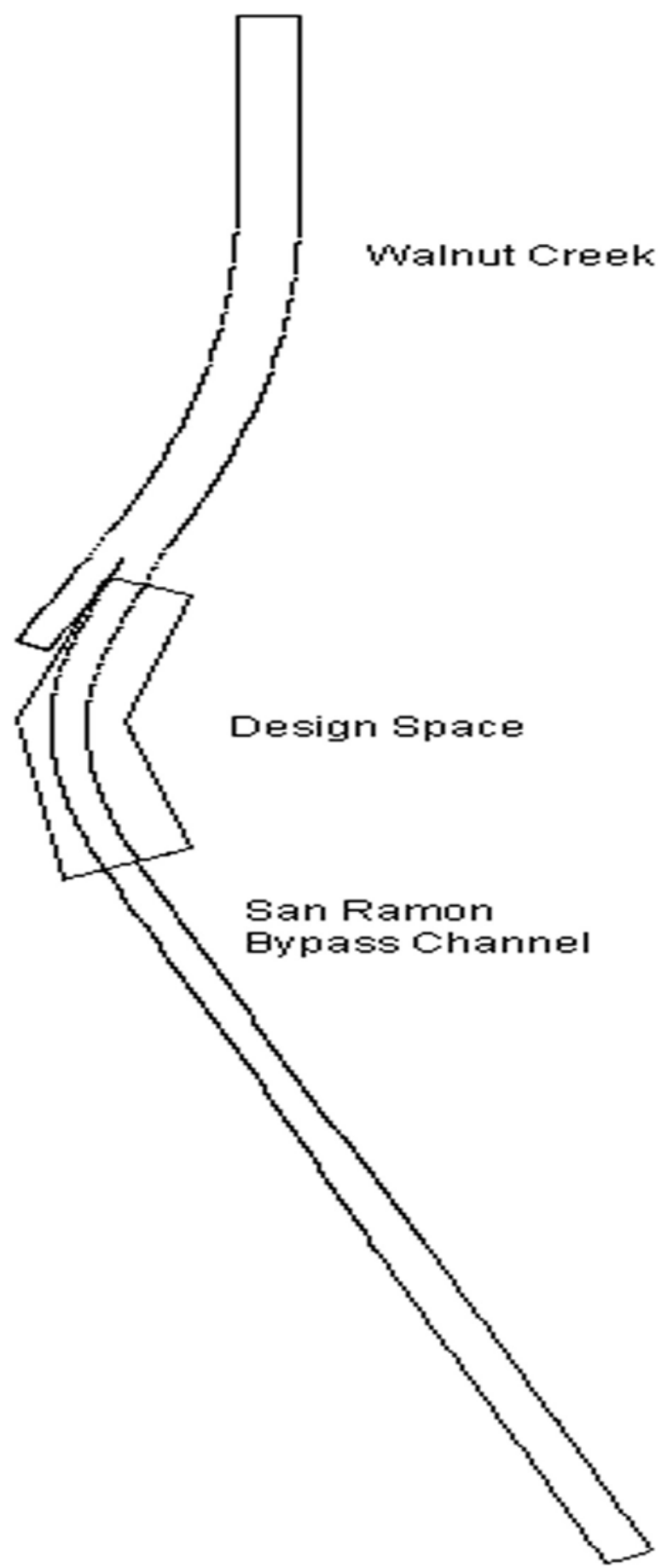

Figure 4.40: Design Space for the Two Design Variable Problem. 


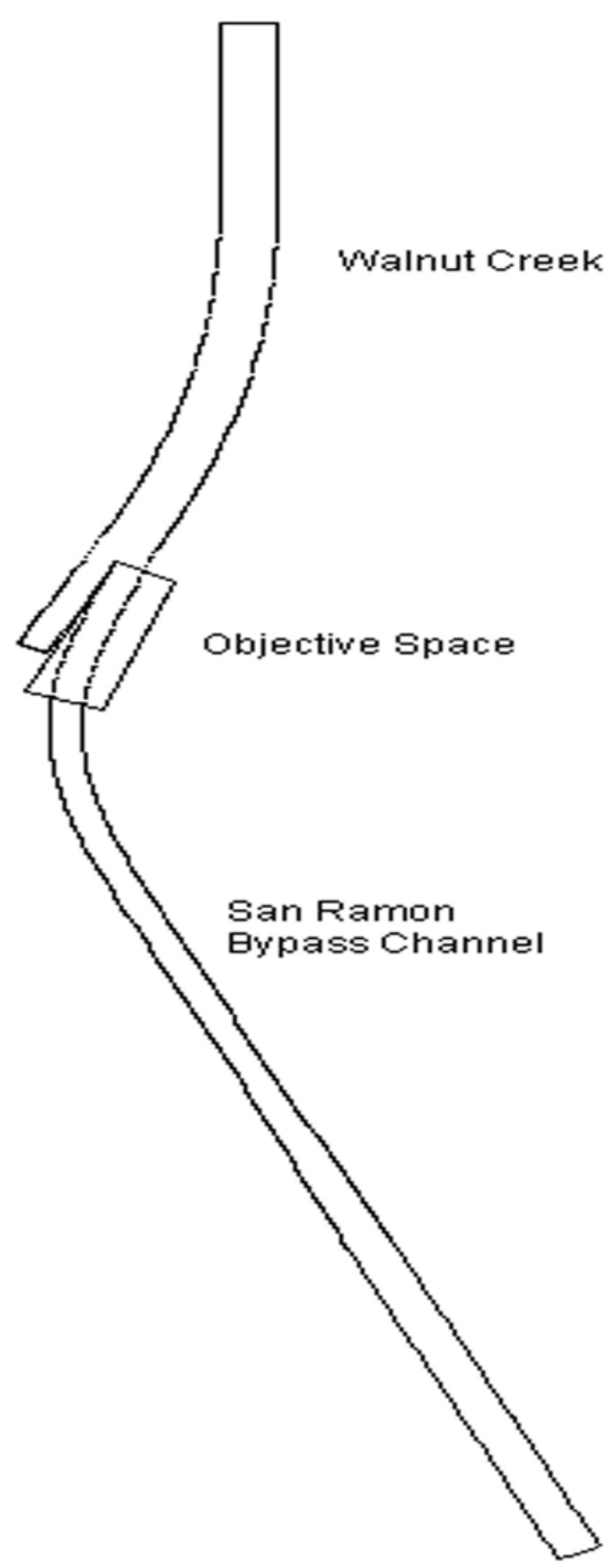

Figure 4.41: The Objective Area for the Two Design Variable Problem. 

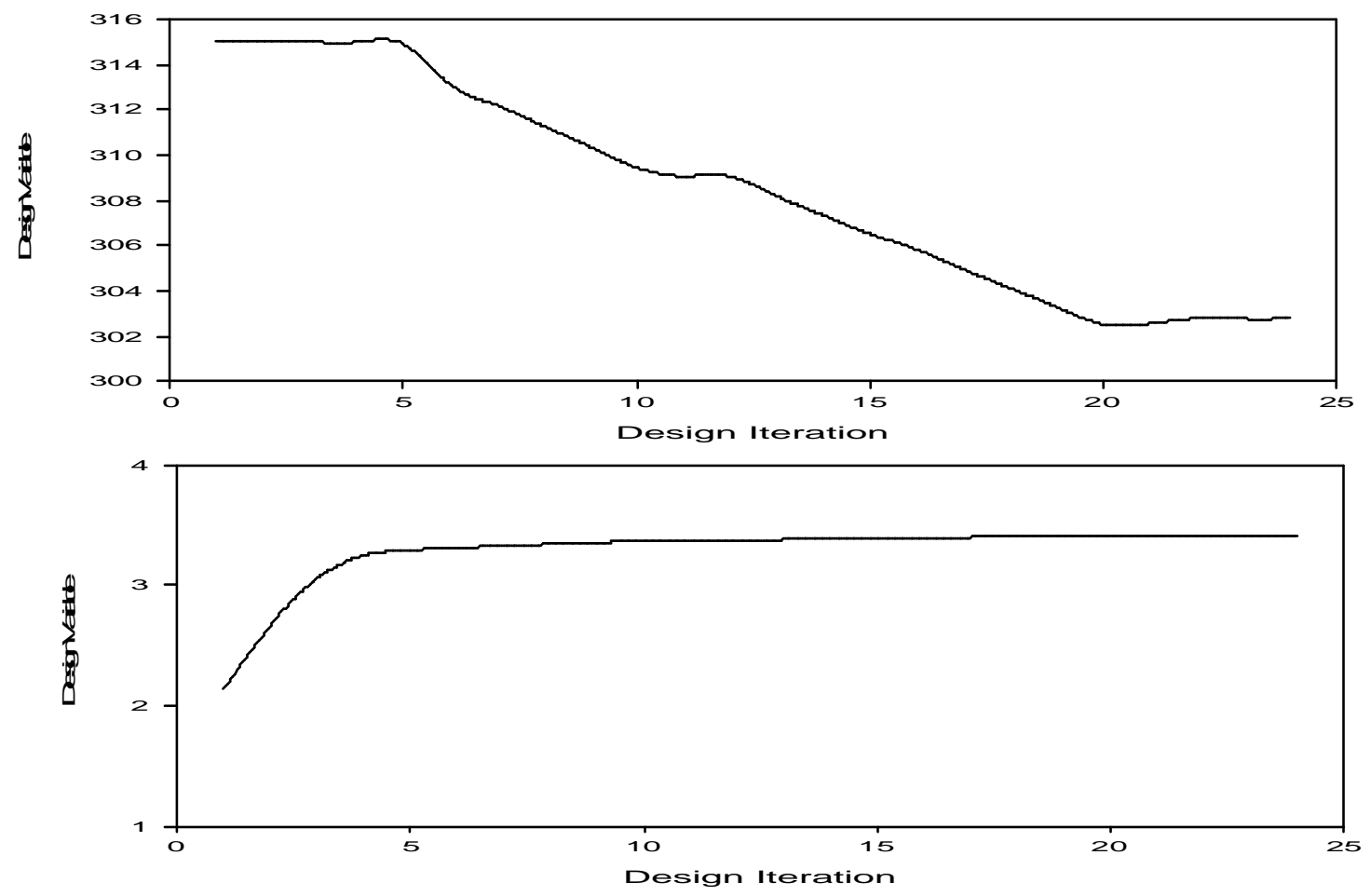

Figure 4.42: Design Variable versus Design Iteration. 


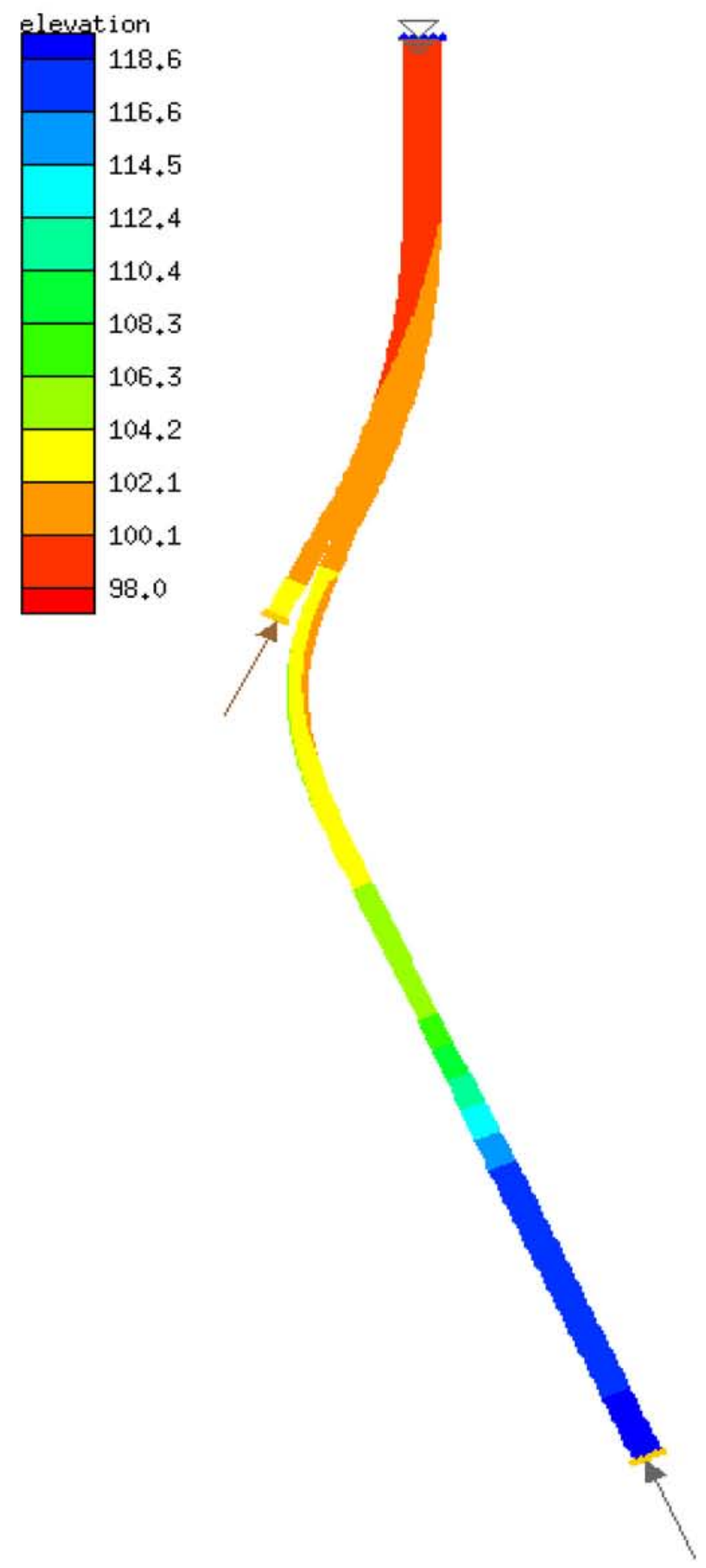

Figure 4.43: Layout of the Improved Design. 


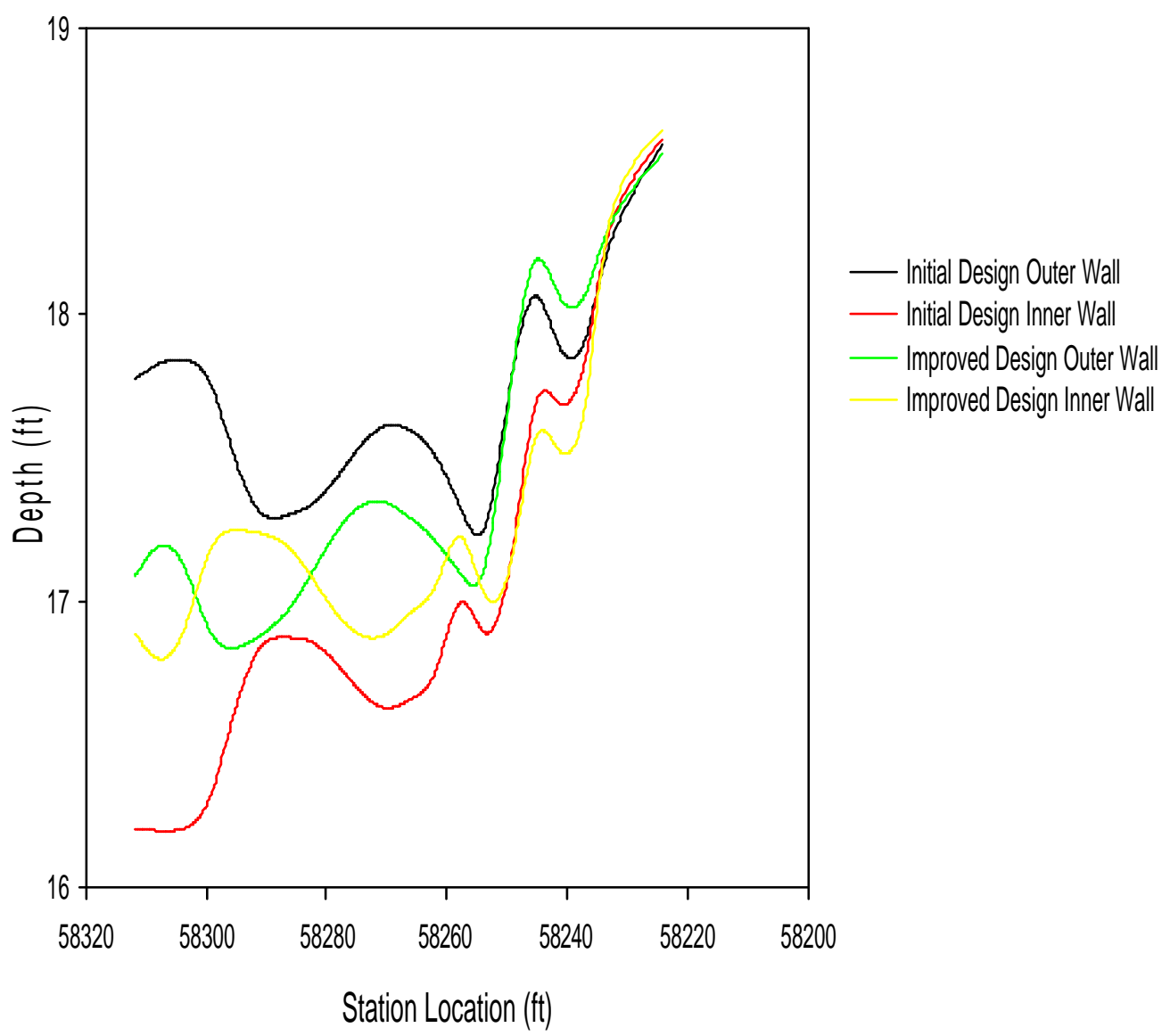

Figure 4.44: Depth Profiles for Initial Design and Improved Design. 


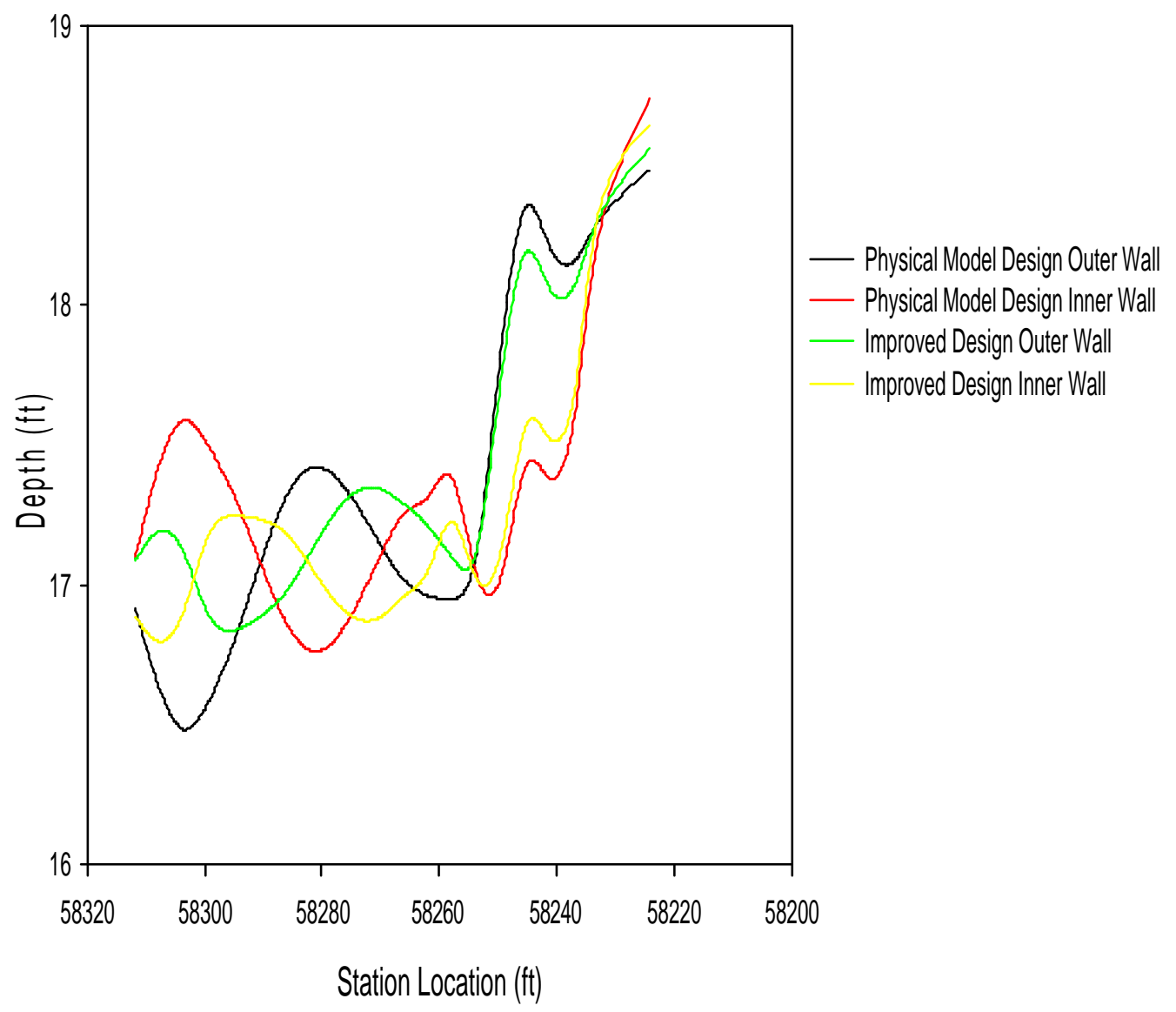

Figure 4.45: Depth Profiles for Physical Model Design and Improved Design. 


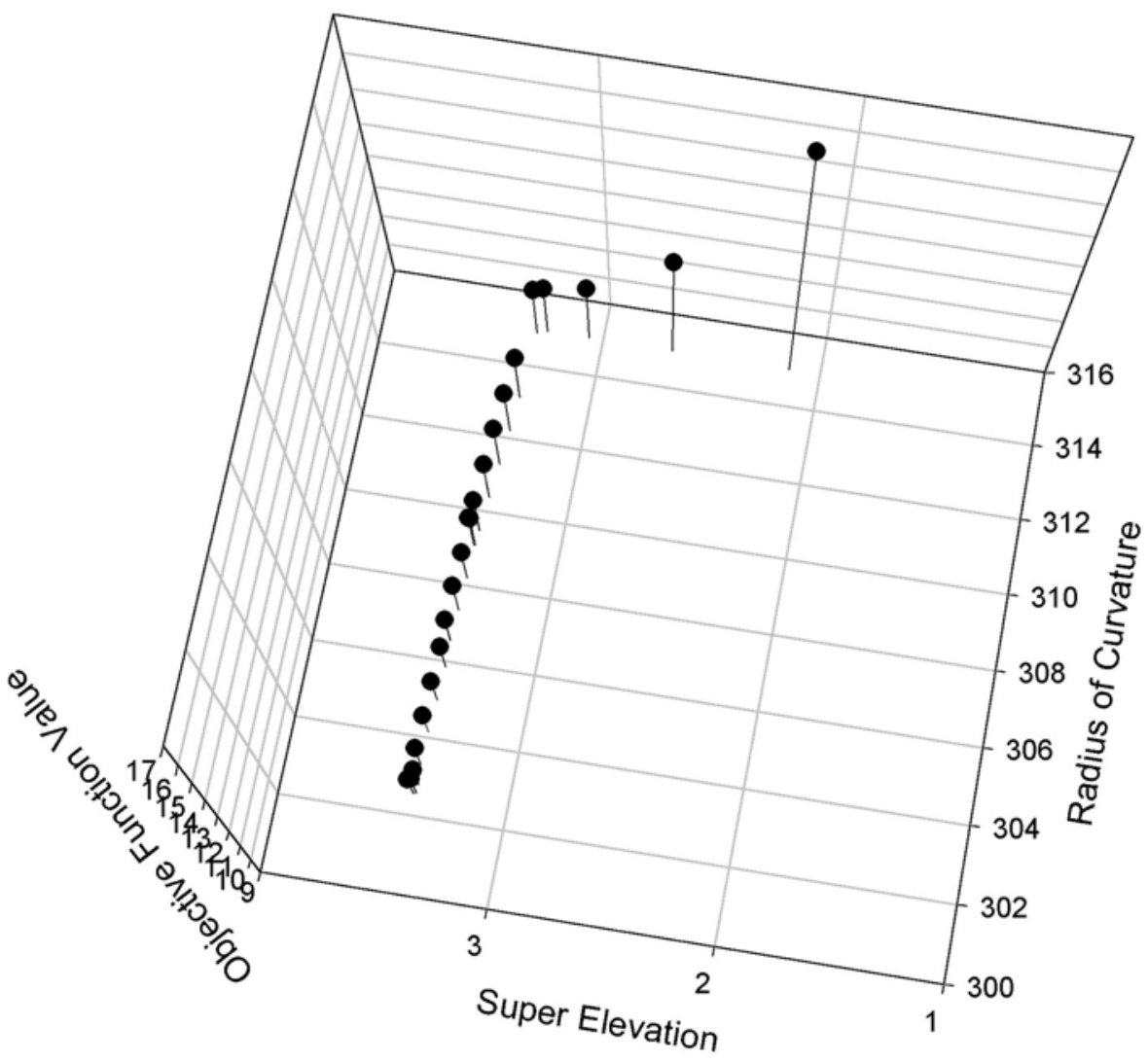

Figure 4.46: Design Variable versus Objective Function. 


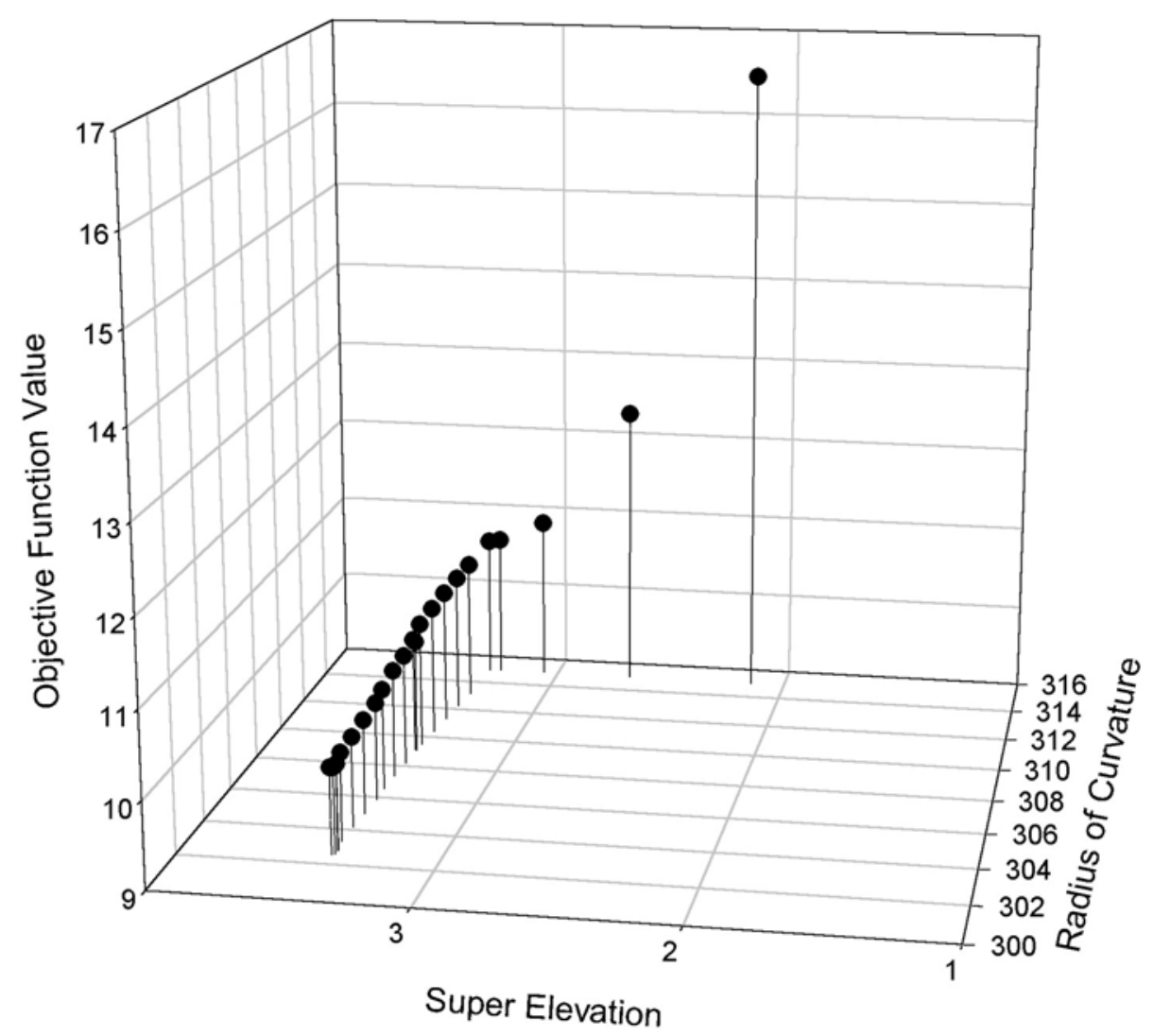

Figure 4.47: Design Variable versus Objective Function. 


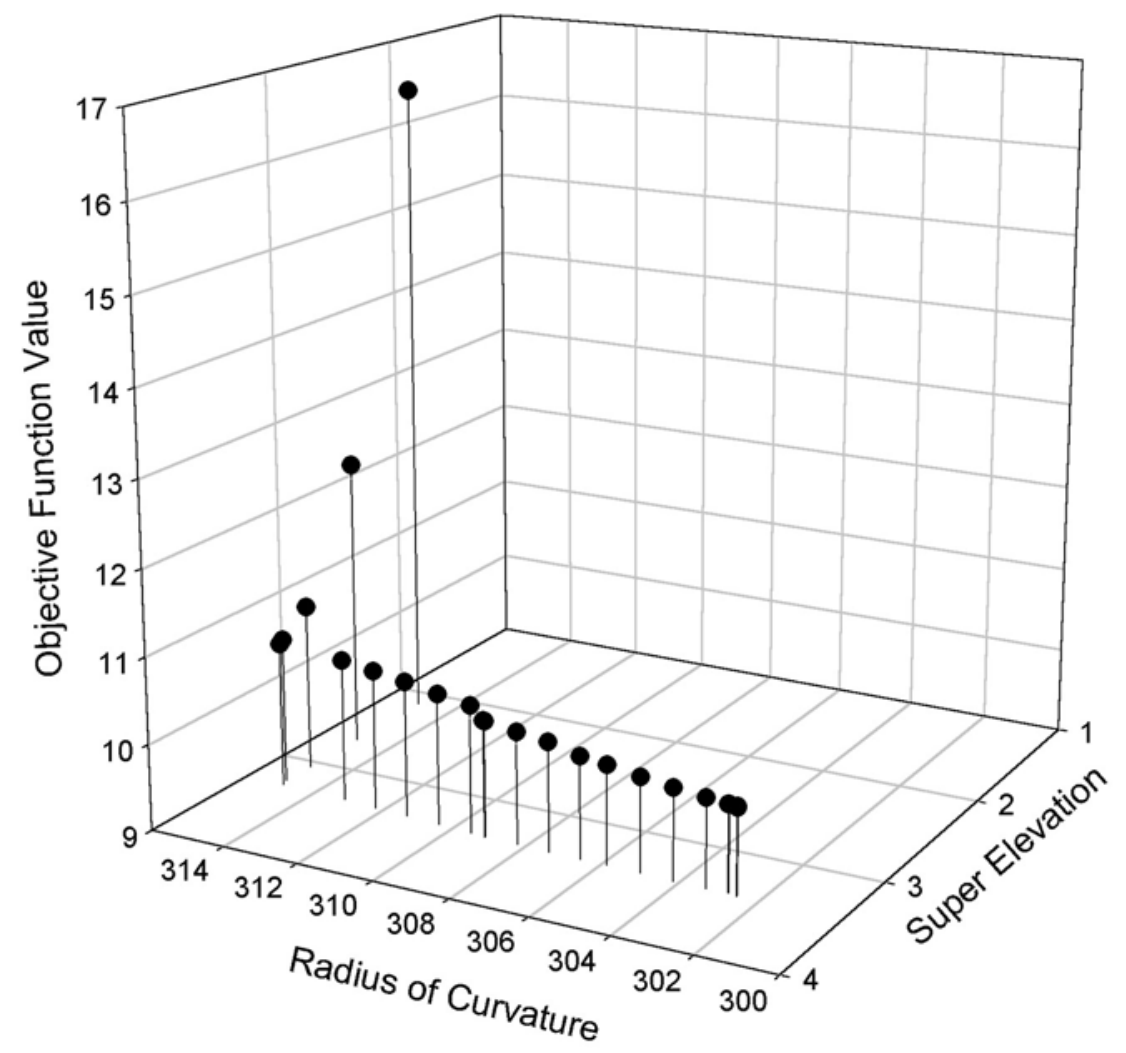

Figure 4.48: Design Variable versus Objective Function. 


\section{CHAPTER V}

\section{SUMMARY, CONCLUSIONS, AND RECOMMENDATIONS FOR FUTURE WORK}

\subsection{Summary}

The capability to use optimization techniques to design "real-world" highvelocity channels has been tested, and the methodology for such an application has been developed. The results show that optimization techniques along with an automated process to re-generate the grid are capable of determining an improved design for high-velocity channels.

Several preliminary steps are necessary before the optimization techniques are applied. Developing a numerical representation of the physical problem is the first preliminary step. After the physical problem is represented numerically, an objective function and the objective space are determined. This is a crucial part of the optimization process, since the objective function must encompass all of the engineering judgment. The objective function in this work measures the non-uniformity of the flow depths. High-velocity channels consist of many design parameters. Some of these parameters have more of an impact on the channel than others. The parameters to which the model is most sensitive can be determined via parameter sensitivity analysis. This allows for limitation of design parameters to those with the greatest impact on the channel and indicates whether sensitivities to the parameters are global or local. 
The application of the optimization techniques to a high-velocity channel requires a mechanism to reflect changes to the design variables in the channel. This is accomplished through an automated grid generation tool. Once the channel design is updated with respect to the design variables, HIVEL2D is used to simulate the steady-state flow conditions. These steady-state conditions are used to determine the objective function value. The objective function value is indicative of the adequacy of the design. The quasi-analytic formulation is used to compute the design space gradients, and the design variables are updated using the Gauss-Newton method. The optimization process is repeated until the model is converged. The convergence criterion is based on the change in the objective function for the current design iteration and the previous design iteration. Convergence of the optimization code yields an improved design.

The super elevation and radius of curvature in the bend just upstream of the Walnut Creek-San Ramon Bypass Channel junction are determined using this optimization technique. Each design parameter has a local effect. That is, changes to the super elevation and radius of curvature in the bend effect the model in the bend and just downstream of the bend and have minimal impact in other areas of the channel. The optimization process is applied to each design variable separately, and then it is applied to both design parameters via two design variable optimization.

\subsection{Conclusions}

The application of the optimization process to the design variables of super elevation and radius of curvature in the bend of San Ramon Bypass Channel yields an improved design. The objective function for the super elevation problem 
is reduced by $30 \%$. A reduction of the objective function of $17 \%$ is achieved in the radius of curvature design problem. By applying the optimization technique to both super elevation and radius of curvature through a multiple design variable process, it is possible to reduce the objective function value by over $40 \%$. As indicated by the objective function, each case provides an improved channel design.

The results show that optimization techniques can be applied to realistic highvelocity channels to produce an improved design. The steps by which such an application is made are developed and outlined in this work. The approach has much utility in high-velocity channel design. Using this technique will allow screening of designs for numerous design parameters. With a more sophisticated automation process, it would be possible to design an entire channel to meet certain constraints and produce a prescribed flow condition. Although this research addressed a two-dimensional problem that solved the shallow water equations, the optimization techniques are not limited to such problems. They can also be applied to various types of flow problems that solve the Navier-Stokes equations and to groundwater flow problems, as well as problems in other engineering disciplines.

\subsection{Recommendations for Future Work}

There are a few areas that can be investigated to expand this technique. Development of an improved automation process is important for expanding the process to different problems and disciplines. The current automation process is designed for the problem investigated in this work. Some effort is required to apply this technique to super elevation and radius of curvature in another

problem. Applying this technique to other design parameters would require a re-evaluation of the automation process. The automation process that would be 
most valuable would be one that has the capability of generating the entire mesh given prescribed parameters such as fixed boundaries, moveable boundaries, and boundary conditions. This could be enhanced via a graphical user interface, which allowed the problem to be defined, launch the optimization technique, and provide real-time updates of the design variables. Such a process would make it easier to apply the optimization techniques to multiple design variables.

The problem examined in this work dealt with the two-dimensional shallow water equations. Additional work should be done to apply these optimization strategies to three-dimensional problems, thus allowing investigation of a wider range of channels. An important issue to remember when applying the optimization techniques to other problems is that the objective function is sitespecific, and the design criteria are different for every problem. Because of these differences, the objective function should be investigated for every application. 


\section{REFERENCES}

[Atanov 98] Atanov, G. A., Evseeva, E. G., and Work, P. A., "Variational Problem of Water-Level Stabilization in Open Channels", Journal of Hydraulic Engineering, Vol. 124, No. 1, January 1998, pp. 50-54.

[Berger 95] Berger, R. C. and Stockstill, R. L., "Finite-Element Model for HighVelocity Channels", Journal of Hydraulic Engineering, Vol. 121, No. 10, Oct. 1995, pp. 710-716.

[Bischof 92] Bischof, C., Carle, A., Corliss, G., Griewank, A., and Hovland, P., "ADIFOR - Generating Derivative Codes from Fortran Programs", Scientific Programming,1(1), pp. 1-29, 1992.

[Broyden] Broyden, C. G., "The Convergence of a Class of Double-Rank Minimization Algorithms", Journal Inst. Maths. Applics., Vol. 6, pp. 76-90.

[Bryson] Bryson, Jr., A. E. and Ho, Y.-C., Applied Optimal Control: Optimization, Estimation, and Control, Halsted Press, New York, 1975.

[Burg 99] Burg, C. O. E., "A Design Optimization Strategy for Open-Channel Flows Using Discrete Sensitivity Analysis", Dissertation, Mississippi State University, August 1999.

[Carey 99] Carey, Graham F., Computational Grids: Generation, Adaptation, and Solution Strategies, Taylor and Francis, Washington, D.C., 1997.

[Cunha 99] Cunha, C. M. and Sousa, J., "Water Distribution Network Design Optimization: Simulated Annealing Approach", Journal of Water Resources Planning and Management, Vol. 125, No. 4, July/August 1999, pp. 215-221.

[Davis 87] Davis, W. G., "Walnut Creek Flood-Control Project Contra Costa County, California Hydraulic Model Investigation", Technical Report HL-8714, WES, October 1987.

[Dennis 87] Dennis, Jr., J. E. and Schnabel, R. B., A View of Unconstrained Optimization, CU-CS-376-87, University of Colorado, 1987. 
[Dowd 93] Dowd, K., High Performance Computing, O’Reilly \& Associates, Inc., California, 1993.

[Farin 93] Farin, G., Curves and Surfaces for Computer Aided Geometric Design, A Practical Guide, 3rd Edition, Academic Press, New York, 1993.

[Geoffrion 72] Geoffrion, A.M. Perspectives on Optimization: A Collection of Expository Articles, 1972.

[Gill 80] Gill, P. E., Murray, W., Suanders, M. A., and Wright, M. H., Aspects of Mathematical Modeling Related to Optimization, TR SOL 80-7, Systems Optimization Laboratory, Stanford University, May 1980.

[Gill 81] Gill, P. E., Murray, W., and Wright, M. H., Practical Optimization, Academic Press, New York, 1981.

[Goldberg 89] Goldberg, D. E., Genetic Algorithms in Search, Optimization and Machine Learning, Addison-Wesley, Reading, MA, 1989.

[Guan 99] Guan, J. and Aral M. M., "Optimal Remediation With Well Locations and Pumping Rates Selected as Continuous Decision Variables", Journal of Hydrology, Vol. 221, 1999, pp. 20-42.

[Guillou 99] Guillou, S. and Nguyen, K. D., "An Improved Technique for Solving Two-Dimensional Shallow Water Problems", International Journal for Numerical Methods in Fluids, Vol. 29, 1999, pp. 465-483.

[Gumprecht 97] Gumprecht, Blake. "51 Miles of Concrete: The exploitation and Transformation of the Los Angeles River", Southern California Quarterly, 79:4, Winter 1997, pp. 431-486.

[Holland 75] Holland, J. H., Adaptation in Natural and Artificial Systems, University of Michigan Press, 1975.

[Hou 94] Hou, G. J.-W., Taylor, III, A. C., and Korivi, V. M., "Discrete Shape Sensitivity Equations for Aerodynamic Problems", International Journal for Numerical Methods in Engineering, Vol. 37, 1994, pp. 2251-2266.

[Hsu 98] Hsu, C.-C., Lee, W.-J., and Chang, C.-H., "Subcritical Open-Channel Junction Flow", Journal of Hydraulic Engineering, Vol. 124, No. 8, August 1998, pp. 847-855.

[HsuM 99] Hsu, M.-H., Kuo, A. Y., Kuo, J.-T., and Liu, W.-C., "Procedure to Calibrate and Verify Numerical Models of Estuarine Hydrodynamics", Journal of Hydraulic Engineering, Vol. 125, No. 2, February 1999, pp. 166-182. 
[Indelman 96] Indelman, P., Dagan, G., Chang, A. H.-D., and Ouazar, D., "Sensitivity Analysis of Flow in Multilayered Leaky Aquifer Systems", Journal of Hydraulic Engineering, Vol. 122, No. 1, Jan. 1996, pp. 41-45.

[Mäkinén 99] Mäkinén, R. A. E., Periaux, J., and Toivanen, J., "Multidisciplinary Shape Optimization in Aerodynamics and Electromagnetics Using Genetic Algorithms", International Journal of Numerical Methods in Fluids, Vol. 30, 1999, pp. 149-159.

[McKinney 94] McKinney, D. C. and Lin, M. D., "Genetic Algorithm Solution of Groundwater Management Problems", Water Resources Research, Vol. 30, No. 6, 1994, pp. 1897-1906.

[Melvin 99] Melvin, R. G., Huffman, W. P., Young, D. P., Johnson, F. T., Hilmes, C. L., and Bieterman, M. B., "Recent Progress In Aerodynamic Design Optimization", International Journal for Numerical Methods in Fluids, Vol. 30, 1999, pp. 205-216.

[Mohammadi 99] Mohammadi, B. and Pironneau, O., "Mesh Adaption and Automatic Differentiation in a CAD-Free Framework for Optimal Shape Design", International Journal for Numerical Methods in Fluids, Vol. 30, 1999, pp. 127-136.

[Mohan 97] Mohan, S., "Parameter Estimation of Nonlinear Muskingum Models Using Genetic Algorithm", Journal of Hydraulic Engineering, Vol. 123, No. 2, February 1997, pp. 137-142.

[Mousavi 00] Mousavi, H. and Ramamurthy, A. S., "Optimal Design of MultiReservoir Systems for Water Supply", Advances in Water Resources, Vol 23, 2000, pp. 613-624.

[Piasecki 97] Piasecki, M. and Katopodes, N. D., "Control of Contaminant releases in Rivers. I: Adjoint Sensitivity Analysis", Journal of Hydraulic Engineering, Vol. 123, No. 6, June 1997, pp. 486-492.

[Reklaitis 83] Reklaitis, G.V., Ravindran, A., and Ragsdell, K.M., Engineering Optimization: Methods and Applications, 1983.

[Ritzel 94] Ritzel, B. J., Eheart, J. W., and Ranjithan, S., "Using Genetic Algorithms to Solve a Multiple Objective Ground Water Pollution Containment Problem", Water Resources Research, Vol. 30, No. 5, 1994, pp. 1589-1603.

[Rubey 38] Rubey, H., Route Surveys: A Pocket Book for the Survey, Design, and Construction of Railways, Highways, Canals, Flumes, Levees, Pipe Lines, 
Transmission Lines, and other Route Constructions, The Macmillan Company, New York, 1938, pp.103-138.

[Sanchez 98] Sanchez, L., Arroyo, V., Garcia, J., Koev, K., and Revilla, J., "Use of Neural Networks in Design of Coastal Sewage Systems", Journal of Hydraulic Engineering, Vol. 124, No. 5, May 1998, pp. 457-464.

[SMS 00] Surface Water Modeling System Reference Manual.

[Soemarwoto 99] Soemarwoto, B. I. and Labrujère, TH. E., "Airfoil Design and Optimization Methods: Recent Progress at NLR", International Journal for Numerical Methods in Fluids, Vol. 30, 1999, pp. 217-228.

[Soulis 92] Soulis, J. V. and Psoni, G. A., "Computer-Aided Design of Channel Expansions and Contractions", International Journal for Numerical Methods in Fluids, Vol. 15, 1992, pp. 1-22.

[Stockstill 00] Communication with Richard Stockstill.

[Storck 97] Storck, P., Eheart, J. W., and Valocchi, A. J., "A Method for the Optimal Location of Monitoring Wells for Detection of Groundwater Contamination in Three-Dimensional Heterogenous Aquifers", Water Resources Research, Vol. 33, No. 9, September 1997, pp. 2081-2088.

[Sun 92] Sun, N.-Z. and Yeh, W. W.-G., "A Stochastic Inverse Solution for Transient Groundwater Flow: Parameter Identification and Reliability Analysis", Water Resources Research, Vol. 28, No. 12, December 1992, pp. 3269-3280.

[Townley 85] Townley, L. R. and Wilson, J. L., "Computationally Efficient Algorithms for Parameter Estimation and Uncertainty Propagation in Numerical Models of Groundwater Flow", Water Resources Research, Vol. 21, No. 12, December 1985, pp. 1851-1860.

[Traub 80] Traub, J. F. and Wozniakowski, H., A General Theory of Optimal Algorithms, Academic Press, New York, 1980.

[EM 1110-2-1601] U.S. Army Corps of Engineers, "Hydraulic Design of Flood Control Channels", Engineer Manual No. 1110-2-1601, 1994.

[Walsh 75] Walsh, G.R. Methods of Optimization, 1975.

[Zhou 99] Zhou, J. G. and Stansby, P. K., "2D Shallow Water Flow Model for the Hydraulic Jump", International Journal for Numerical Methods in Fluids, Vol. 29, 1999, pp. 375-387. 
[Zhu 99] Zhu, J., Zeng, Q., Guo, D., and Liu, Z., "Optimal Control of Sedimentation in Navigation Channels", Journal of Hydraulic Engineering, Vol. 125, No. 7, July 1999, pp. 750-759. 
APPENDIX

OPTIMIZATION 
Engineers are sometimes required to select the "best" design from a set of feasible designs. This is called optimal design or optimization. Optimization principles are of increasing importance in modern design. Even in standard applications, much is to be gained by using an algorithm to generate initial meshes and refine to a"near-optimal" mesh automatically.

For this work optimization is generally defined as follows. Given a function of one or more independent variables, find the value of those variables where $\mathrm{F}$ is a minimum. The function $\mathrm{F}$ should give an objective measure of the quality of the system state. Minimizing F should be accomplished using as few calculations of $\mathrm{F}$ as possible due to the propensity of the calculations to be computationally expensive. Values of the variables are calculated using iterative processes that start at some point and move stepwise to points for which $\mathrm{F}$ is smaller. There are many different means of calculating the next guess for the variables. Two major methods are heuristic methods and gradient-based methods. The influence coefficients are calculated via sensitivity analysis.

\section{Heuristic Method}

Heuristic search techniques, such as genetic algorithms, simulated annealing, and neural networks achieve nearly optimal solutions at a reasonable computational cost, without guaranteeing a globally optimal solution will be found. These techniques are stochastic search procedures that use probabilistic rather than deterministic search rules. Genetic algorithms are search algorithms based on the concept of natural selection and natural genetics [Holland 75]. The objective function magnitude, instead of derivative information, is used directly in the search, therefore allowing these techniques to be applied to nonconvex, highly nonlinear 
and complex problems [Goldberg 89]. Techniques of this type can locate nearoptimal solutions of complex problems with very large nonlinear search spaces. Discontinuous fitness functions and discrete input variables may be used. Genetic algorithms do not fall into local minima easily, and the configuration decisions proceed in logical order [Cunha 99]. These techniques can be applied to a wide range of problems including scheduling and networking. "Good" solutions to highly complex problems can be found within a reasonable amount of time. Methods like genetic algorithms lend themselves to parallel application due to the need to evaluate each member of the population at each generation.

Advantages of heuristic techniques lie in their ability to locate solutions to combinatorial optimization problems with greater efficiency than implicit enumerations techniques. Moreover, these techniques more easily accommodate the discontinuities and non-linearities of real-world problems than do gradientbased techniques [McKinney 94][Ritzel 94]. A disadvantage of these techniques are the need for large populations to be evaluated over many generations. In computational fluid dynamics, the cost of analyzing the design for a numerical simulation can be expensive, therefore genetic algorithms are usually used with less computationally expensive, lower fidelity models [Burg 99].

\section{Gradient-based Method}

Gradient-based methods are conceptually simple and deterministic. Many optimization algorithms use gradients. Gradient-based methods use both first derivative (gradient) information and second derivative (Hessian) information and are based on the simple fact that $f(x)$ increases or decreases in the direction $\mathbf{d}$ according as the directional derivative $[\nabla F(x)]^{\prime} d$ is positive or negative. That is, 
the fastest way to find an extremum is to move along the gradient. Obtaining these derivatives, however, can be expensive, and the information may be inaccurate. There are several gradient-based methods. Some of the more popular ones are the method of steepest descent, conjugate gradient methods, Newton's method, quasi-Newton method, trust region models, and Gauss-Newton method.

The method of steepest descent is often used to calculate the next guess $\bar{x}^{n+1}$ when the only available information is $F\left(\bar{x}^{n}\right)$ and $\nabla F\left(\bar{x}^{n}\right)$. The primary concept is that the optimal search direction $p^{n}$ at $x^{n}$ is the gradient or steepest descent direction $\nabla F\left(\bar{x}^{n}\right)$, therefore the value of $F\left(\bar{x}^{n}\right)$ is decreased by moving in this direction with small step sizes. Drawbacks to this method are the need for one or more additional function evaluations per design iteration and the linear convergence rates. Also, due to poor convergence, this method may not be efficient for realistic design optimization algorithms as many design iterations may be required. The gradient of the function is defined as follows.

$$
\nabla f_{k}(x) \approx \frac{\partial f(x)}{\partial x_{k}}, 1 \leq k \leq n
$$

If $\alpha_{k}$ denotes the optimal step length resulting from searching along the direction $d^{k}=-\nabla f\left(x^{k}\right)$ starting from the point $x^{k}$, then the updated estimate of the solution is computed as follows.

$$
x^{k+1}=x^{k}-\alpha_{k} \nabla f\left(x_{k}\right), k \geq 0
$$


If the expression for the gradient is available, then the derivative of $F(\alpha)$ needed for the bisection method can be computed using the chain rule as follows.

$$
F^{\prime}(\alpha)=\left(d^{k}\right)^{T} \nabla f\left(x^{k}+\alpha d^{k}\right)
$$

The conjugate gradient method uses $F\left(\bar{x}^{n}\right)$ and $\nabla F\left(\bar{x}^{n}\right)$ like the steepest descent method but also has an additional constraint. The current search direction, $p^{n}$, must be conjugate, or perpendicular, to the previous design variable search directions. For quadratic functions, the conjugate gradient method identifies the optimal solution exactly within the number of design variable iterations. Drawbacks to this method are that optimal step size is required to achieve efficient convergence; therefore multiple function evaluations are necessary to determine optimal step size. Also, for design optimization using high-fidelity simulation, the conjugate gradient method is infeasible due to the high computational cost of the function evaluation. The convergence rate scales with the number of design variables [Burg 99].

Newton's method is another gradient-based method. It uses the Hessian to update the design variable. This method generally yields quadratic convergence once the iterates have moved within the convergence region. A drawback to this method is the need for the Hessian matrix. This method is rarely used.

For highly nonlinear functions, the trust region model uses the knowledge that the best search direction as the step size tends towards zero is the gradient direction. The search direction is approximated as a combination of the gradient direction and the Newton search direction. This method has received much attention as a means to improve the convergence of implicit flow solvers. 
Drawbacks to this method are the computational cost of the function evaluations and the need to calculate the Hessian matrix.

The quasi-Newton method gradually builds an approximation to the Hessian matrix based on information at the previous iterations and uses the Hessian to determine the search direction and step size. A requirement for this method is that the Hessian matrix remain symmetric and positive definite and that the new Hessian satisfy the secant equation $B_{n+1} s_{n}=y_{n}$. The "best" update formula for this method is the Broyden-Fletcher-Goldfarb-Shanno (BFGS) according to Broyden [Broyden]. These update methods are often used to achieve super-linear convergence rates.

The Gauss-Newton method approximates the Hessian by assuming the second order term in $\nabla^{2} F$ is negligible. This assumption restricts the objective function to a least-squares function. That is, given a function

$$
\begin{gathered}
F(\bar{x})=\sum_{i=1}^{N} r_{i}^{2}(\vec{x})=R^{T}(\vec{x}) R(\vec{x}) \\
r_{i}(\vec{x})=\text { N residual functions } \\
\vec{x}=\text { a vector of variables } \\
R(\vec{x})=\text { a vector of residual functions }
\end{gathered}
$$

This method uses the structure of the function $F(\vec{x})$ to approximate the Hessian matrix. Hence, it is able to produce super-linear convergence. The first derivative of the function is

$$
\frac{\partial F}{\partial x_{k}}=\sum_{i=1}^{N} 2 \frac{\partial r_{i}}{\partial x_{k}} r_{i}=2 \frac{\partial R^{T}}{\partial x_{k}} R
$$


and the gradient is

$$
\nabla F(\vec{x})=2 \nabla R^{T} R
$$

The second derivative is

$$
\frac{\partial^{2} F}{\partial x_{k} x_{j}}=\sum_{i=1}^{N} 2 \frac{\partial r_{i}^{T}}{\partial x_{k}} \frac{\partial r_{i}}{\partial x_{j}}+\sum_{i=1}^{N} 2 \frac{\partial^{2} r_{i}}{\partial x_{k} \partial x_{j}} r_{i}=2 \frac{\partial R_{T}}{\partial x_{k}} \frac{\partial R}{\partial x_{j}}+2 \frac{\partial^{2} R^{T}}{\partial x_{k} \partial x_{j}} R
$$

and the Hessian Matrix is

$$
\nabla^{2} F(\vec{x})=x \nabla R^{T} \nabla R+2 S
$$

where $\mathrm{S}$ is a matrix whose entries are $\frac{\partial^{2} R^{T}}{\partial x_{k} \partial x_{j}} R$. The term $\mathrm{S}$ is assumed to be relatively small compared to $\left(\nabla R^{n}\right)^{T} \nabla R^{n}$ near the local extrema, hence it can be ignored. Using this assumptions allows for $\vec{p}^{n}$ to be obtained via

$$
2\left(\nabla R^{n}\right)^{T} \nabla R^{n} \triangle \vec{p}^{n}=-2\left(\nabla R^{n}\right)^{T} R^{n}
$$

Burg notes that though the assumptions made do not apply to his work, the assumption that $\mathrm{S}$ can be ignored appears to be reasonable, since the GaussNewton method obtained good design improvement results [Burg 99].

\section{Sensitivity Analysis}

Estimating the design space gradient can be computationally expensive because it would require an additional steady state calculation for each design variable. Using sensitivity analysis can reduce this cost. The design space gradient is estimated by using information gained from differentiating the system of governing 
equations. There are two basic types of sensitivity analysis - the discrete approach and the continuum approach. The discrete approach takes analytical derivatives of the discretized equations with respect to the independent variables $(\mathrm{Q}, \chi, \beta)$. The continuum approach calculates derivatives directly, based upon the continuous governing equations, by using the method of material derivatives. The governing equations are discretized and then differentiated with the discrete approach. In the continuous approach the governing equations are differentiated and then discretized.

Discrete sensitivity analysis derives sensitivity equations by differentiating the discretized system of governing partial differential equations (which involves the Jacobian). Many implicit simulation models use this same Jacobian matrix, and the matrix equation solution subroutines can be used for the analysis. Explicit codes do not use the Jacobian. Therefore, it must be generated for sensitivity analysis. The complex Taylor's series expansion (CTSE) can be used to generate Jacobian matrix from the discretized system of equations when the Jacobian does not already exist. The CTSE can be used to generate numerically exact Jacobians for the implicit codes as well.

The continuous approach, also known as the continuous adjoint approach, uses variational calculus to derive the adjoint equations. The objective function $F$, the governing partial differential equations $P$, and the boundary conditions are functions of the flow variables $Q$, the computational domain or grid $\chi$, and the design variables. Forming the Lagrangian, taking the variation, and satisfying the governing equations for a steady state problem yields 


$$
\delta L=\frac{\partial L}{\partial \chi} \delta \chi+\sum_{i} \frac{\partial L}{\partial \beta_{i}} \delta \beta_{i}
$$

or

$$
\begin{aligned}
\frac{\delta L}{\delta \beta_{i}} & =\frac{\partial L}{\partial \chi} \frac{\delta \chi}{\delta \beta_{i}}+\frac{\partial L}{\partial \beta_{i}}=\left.\frac{d L}{d \beta_{i}}\right|_{Q \text { fixed }} \\
& \approx \frac{L\left(Q(\vec{\beta}), \chi\left(\vec{\beta}+e_{i} \Delta \beta_{i}\right), \vec{\beta}+e_{i} \Delta \beta_{i}, \lambda_{j}, \Gamma_{k}\right)-L\left(Q(\vec{\beta}), \chi(\vec{\beta}), \vec{\beta}, \lambda_{j}, \Gamma_{k}\right)}{2 \Delta \beta_{i}}
\end{aligned}
$$

Since the partial differential equations and the boundary conditions are satisfied

$$
\frac{d F}{d \vec{\beta}_{i}}=\left.\frac{d L}{d \vec{\beta}_{i}}\right|_{Q f i x e d}
$$

With the continuous approach a system of partial differential equations is differentiated to yield a system of adjoint equations, which is solved in addition to the governing equations. The difficulties with this method are the need to rewrite analysis code to solve a different set of partial differential equations, the computational cost of solving the adjoint equations, and the non-trivial derivations of the adjoint equations. In addition, the adjoint variable equations must be rederived when changes are made to the math model [Burg 99].

Following Burg [Burg 99], the discrete approach, or direct differentiation, solves a discretized system of equations resulting from the governing partial differential equations being differentiated at each node in the computational domain and a linear matrix equation. The design space derivative of the objective function, 
given by $F(Q(\vec{\beta}), \chi(\vec{\beta}), \vec{\beta})$, is

$$
\frac{d F}{d \beta_{i}}=\frac{\partial F^{T}}{\partial Q} \frac{\partial Q}{\partial \beta_{i}}+\frac{\partial F^{T}}{\partial \chi} \frac{\partial \chi}{\partial \beta_{i}}+\frac{\partial F}{\partial \beta_{i}}
$$

In this equation $\frac{d F}{d \beta_{i}}$ is the total variation of $F$ with respect to the design variable $\beta_{i}$. $\frac{\partial F}{\partial Q}, \frac{\partial F}{\partial \chi}$, and $\frac{\partial F}{\partial \beta_{i}}$ can be calculated since the dependency of $\mathrm{F}$ on $\mathrm{Q}, \chi$, and $\vec{\beta}$ is explicit. $\frac{\partial \chi}{\partial \beta_{i}}$ can be estimated by finite differencing the results of the grid generation code or by differentiating the explicit dependencies between the grid and the design variables with hand-differentiation, complex Taylor series expansion, or ADIFOR [Bischof 92]. Estimation of the vector $\frac{\partial Q}{\partial \beta_{i}}$ with finite differences would require an additional steady-state simulation. This term also appears in the equation for the derivative of the discretized system of governing equations $W(Q(\vec{\beta}), \chi(\vec{\beta}), \vec{\beta})=0$ or

$$
0=\frac{d W}{d \beta_{i}}=\frac{\partial W}{\partial Q} \frac{\partial Q}{\partial \beta_{i}}+\frac{\partial W}{\partial \chi} \frac{\partial \chi}{\partial \beta_{i}}+\frac{\partial W}{\partial \beta_{i}}
$$

As in equation A.13, all terms except for $\frac{\partial Q}{\partial \beta_{i}}$ can be calculated without the need for a steady-state simulation. Equation A.14 along with A.13 are used to estimate the design space derivative $\frac{d F}{d \beta_{i}}$.

For the adjoint variable formulation of discrete sensitivity analysis, equation (A.14) is multiplied by an adjoint vector $\lambda$ and added to equation (A.13). This yields

$$
\frac{d F}{d \beta_{i}}=\left(\frac{\partial F^{T}}{\partial Q}+\lambda^{T} \frac{\partial W}{\partial Q}\right) \frac{\partial Q}{\partial \beta_{i}}+\left(\frac{\partial F^{T}}{\partial \chi}+\lambda^{T} \frac{\partial W}{\partial \chi}\right) \frac{\partial \chi}{\partial \beta_{i}}+\frac{\partial F}{\partial \beta_{i}}+\lambda^{T} \frac{\partial W}{\partial \beta_{i}}
$$


The coefficient of $\frac{\partial Q}{\partial \beta_{i}}$ is set equal to zero to avoid having to calculate for $\frac{\partial Q}{\partial \beta_{i}}$. That is

$$
\begin{aligned}
& \frac{\partial F^{T}}{\partial Q}+\lambda^{T} \frac{\partial W}{\partial Q}=0 \\
& \frac{\partial W^{T}}{\partial Q} \quad \lambda=-\frac{\partial F}{\partial Q}
\end{aligned}
$$

$\lambda$ must be calculated only once for each objective function and is independent of the design variables for the adjoint variable formulation. The adjoint variable formulation scales with the number of functions and constraints and is appropriate for problems with only one objective function and a few constraints. The direct formulation scales with the number of design variables.

For the direct formulation of discrete sensitivity analysis, or quasi-analytic method, the vector $\frac{\partial Q}{\partial \beta_{i}}$ is obtained by solving equation (A.14) directly or

$$
\frac{\partial W}{\partial Q} \frac{\partial Q}{\partial \beta_{i}}=-\frac{\partial W}{\partial \chi} \frac{\partial \chi}{\partial \beta_{i}}-\frac{\partial W}{\partial \beta_{i}}=-\left.\frac{d W}{d \beta_{i}}\right|_{Q f i x e d}
$$

The vector $\frac{\partial Q}{\partial \beta_{i}}$ must be calculated for each design variable. This makes the computational cost of the direct formulation greater than that of the adjoint variable formulation when the number of design variables is greater than the number of objective function and constraints. However, when using a least-squares objective function and the Gauss-Newton optimization algorithm the number of objective functions to be differentiated is much larger than the number of design variables since this algorithm views each term in the objective function as a separate residual function. For this reason, the quasi-analytic method is more cost effective. 
The advantages of the discrete sensitivity analysis are that few modifications are needed for implicit codes. The flow variables are driven to steady-state, and the flow solver, the Jacobian matrix $\frac{\partial W}{\partial Q}$, and the matrix inverse routines are available for implicit codes. Also, the computational cost for discrete sensitivity analysis is smaller than for the continuous approach due to the need to perform the pertinent calculations only at steady-state. A disadvantage of the discrete sensitivity analysis is the need to generate highly accurate Jacobian matrices. Also, most implicit flow solvers only approximate the Jacobian matrix by neglecting friction and turbulence models, making assumptions about upwinding methods and using first order schemes [Piasecki 97]. For explicit solution methods, the Jacobian matrix is not computed as part of the simulation and is unavailable for discrete sensitivity analysis. The Jacobian matrix must be computed.

In his work, Burg discusses accuracy issues related to the discrete approach. He notes that error can be introduced into the calculations of the design space derivatives via several terms. The partial derivatives of the objective function $\mathrm{F}$, the Jacobian matrix $\frac{\partial W}{\partial Q}$, and the vector $\left.\frac{d W}{D \beta_{i}}\right|_{Q \text { fixed }}$ must be determined. Also, the discrete approach assumes that the residual vector $W(Q)$ has been successfully driven to zero and that the matrix inversion operation is highly accurate. This may not be true if the iterative solvers are prematurely terminated. Burg's work uses hand-differentiation to calculate the partial derivative of the objective function $\mathrm{F}$ and the complex Taylor's series expansion to calculate the Jacobian matrix. Burg also notes that the choice of grid and solution methods for the continuous adjoint approach affects the accuracy of the adjoint variable $\lambda$. As a result, the accuracy of the design space derivative is affected. [Burg 99] 


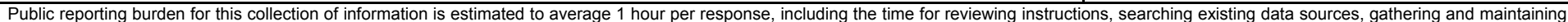

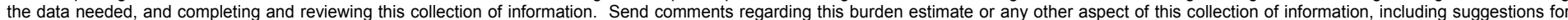

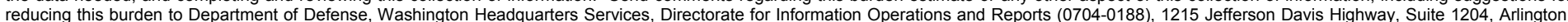

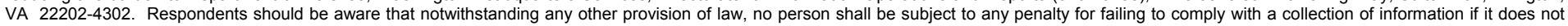
display a currently valid OMB control number. PLEASE DO NOT RETURN YOUR FORM TO THE ABOVE ADDRESS.

\begin{tabular}{l|c}
$\begin{array}{l}\text { 1. REPORT DATE }(D D-M M-Y Y Y Y) \\
\text { May } 2006\end{array}$ & $\begin{array}{c}\text { 2. REPORT TYPE } \\
\text { Final report }\end{array}$ \\
\hline
\end{tabular}

4. TITLE AND SUBTITLE

Automated Design of a High-Velocity Channel

3. DATES COVERED (From - To)

5a. CONTRACT NUMBER

5b. GRANT NUMBER

5c. PROGRAM ELEMENT NUMBER

\section{AUTHOR(S)}

Jacqueline P. Hallberg 5d. PROJECT NUMBER

5e. TASK NUMBER

5f. WORK UNIT NUMBER

8. PERFORMING ORGANIZATION REPORT NUMBER

ERDC/CHL TR-06-2

U.S. Army Engineer Research and Development Center

Coastal and Hydraulics Laboratory

3909 Halls Ferry Road

Vicksburg, MS 39180-6199

9. SPONSORING / MONITORING AGENCY NAME(S) AND ADDRESS(ES)

10. SPONSOR/MONITOR'S ACRONYM(S)

Headquarters, U.S. Army Corps of Engineers

Washington, DC 20314-1000

11. SPONSOR/MONITOR'S REPORT NUMBER(S)

\section{DISTRIBUTION / AVAILABILITY STATEMENT}

Approved for public release; distribution is unlimited.

\section{SUPPLEMENTARY NOTES}

\section{ABSTRACT}

Engineering design is a decision-making process. Optimization techniques can be used to insure that better decisions are made. One design of great interest to engineers is that of high-velocity channels used for routing floodwater out of urban areas. In the design of these channels it is very important to avoid such hydraulic phenomena as standing waves, hydraulic jumps, and shocks. These will require higher wall heights and more expense. These channels can be modeled with physical models, but they are expensive and time consuming. To minimize the cost of building and changing the physical models and the time required to perform the study, an automated numerical model can be used to test a range of designs before construction of the physical model. The resulting design can be used as an initial design, which is close to the desired design requiring fewer changes to the physical model, saving time and money.

\section{SUBJECT TERMS}

Adjoint variable formulation Gauss-Newton

16. SECURITY CLASSIFICATION OF:

a. REPORT

UNCLASSIFIED

\section{b. ABSTRACT} UNCLASSIFIED
High-velocity channels HIVEL2D
Optimization

Quasi-analytic method

System-Wide Water Resources Program SWWRP

17. LIMITATION OF ABSTRACT c. THIS PAGE

UNCLASSIFIED
18. NUMBER OF PAGES

136 PERSON

19b. TELEPHONE NUMBER (include area code) 